\title{
Geometric structure and mechanical stability of disordered tetrahedra packings
}

An experimental X-ray computed tomography study

\author{
Dissertation \\ zur Erlangung des mathematisch-naturwissenschaftlichen \\ Doktorgrades \\ "Doctor rerum naturalium" \\ an der Georg-August-Universität Göttingen \\ im Promotionsprogramm ProPhys \\ der Georg-August-University School of Science (GAUSS)
}

\author{
vorgelegt von \\ Max Neudecker \\ aus Kassel
}

Göttingen, 2013 


\section{Betreuungsausschuss}

Dr. Matthias Schröter (MPIDS Göttingen)

Prof. Dr. Annette Zippelius (Univ. Göttingen)

Prof. Dr. Stephan Herminghaus (MPIDS Göttingen)

\section{Prüfungskommission}

Referentin: Prof. Dr. Annette Zippelius

Koreferent: Prof. Dr. Stephan Herminghaus

\section{Weitere Mitglieder der Prüfungskommission}

Dr. Claus Heussinger (Univ. Göttingen)

Dr. Eleni Katifori (MPIDS Göttingen)

Prof. Dr. Marcus Müller (Univ. Göttingen)

Prof. Dr. Ulrich Parlitz (Univ. Göttingen) 


\section{Contents}

1 Introduction $\quad 7$

1.1 Mechanical stability of granulates . . . . . . . . . . 8

1.1.1 Jamming of spheres . . . . . . . . . . . . . 9

1.1.2 Jamming of tetrahedra . . . . . . . . . . . . 11

1.2 Statistical mechanics of granular matter . . . . . . . . 12

1.2.1 Voronoi volumes . . . . . . . . . . . . . 12

1.3 Packing problems . . . . . . . . . . . . . . . . . 12

1.3.1 Tetrahedral packaging . . . . . . . . . . . . 12

1.3.2 From Hilbert to the New York Times: Pursuing the perfect packing . . . . . . . . . . . . 13

1.3.3 Characterization of order . . . . . . . . . 16

1.3.4 Perfect vs. physical tetrahedra . . . . . . . . . 17

1.4 Geometry of platonic bodies . . . . . . . . . . . . 18

2 Experimental setup $\quad 21$

2.1 Particle characterization . . . . . . . . . . . . . . 21

2.2 Packing preparation - shaken, not stirred . . . . . . . 21

2.2.1 Electromagnetic vibration exciter (Shaker) . . . . . 22

2.2 .2 Laser height sensor . . . . . . . . . . . . . . . 23

2.2.3 Preparation protocols . . . . . . . . . . 25

2.2.4 Initial (loose) preparation . . . . . . . . . . . 25

2.2.5 Compaction by tapping (TAP) . . . . . . . . 25

2.2.6 Annealing procedure (RAMP) . . . . . . . . 26

2.2 .7 Vibration protocol (VIB) . . . . . . . . . . 27

2.3 X-ray tomography . . . . . . . . . . . . . . . . 28

2.3.1 Nanotom setup . . . . . . . . . . . . . . 28

2.3.2 Choice of optimal tomography parameters . . . . . . 28

3 Processing of tomographic volumes 35

3.1 Particle detection . . . . . . . . . . . . . 36

3.1 .1 Algorithm overview . . . . . . . . . . . 36

3.1 .2 Preprocessing and filtering . . . . . . . . 36

3.1.3 Grey-scale based segmentation . . . . . . . . . 38 
3.1.4 Region-based segmentation . . . . . . . . . . . 42

3.1 .5 Object registration . . . . . . . . . . . . 46

3.1 .6 Performance . . . . . . . . . . . . . 52

3.1.7 Future extensions, appendix . . . . . . . . . 56

3.2 Determination of contact numbers . . . . . . . . . . 57

3.2.1 Estimation of error bars . . . . . . . . . . 58

3.3 From contacts to constraints . . . . . . . . . . . 59

3.3.1 Analysis of the contact geometry . . . . . . . . 60

3.3.2 Threshold choice for $\mathrm{FF}$ and $\mathrm{EF}$ contacts . . . . . . 61

3.4 Analysis of Voronoi volumes . . . . . . . . . . . . . . . 62

4 Results and Discussion $\quad 65$

4.1 Protocol dependency of packing fractions . . . . . . . . 66

4.1 .1 Initial preparation . . . . . . . . . . . . 66

4.1 .2 Protocol TAP $(\Gamma=2) \ldots \ldots \ldots 6$

4.1.3 Annealing procedure (RAMP) . . . . . . . . . 67

4.1.4 Protocol dependency of $\Phi_{\infty} \ldots \ldots \ldots$. . . . . . . 68

4.1.5 Vibration protocol (VIB) . . . . . . . . . . . 70

4.1.6 Comparison to previous work . . . . . . . . . . 70

4.1.7 Spatial distribution of $\phi_{\text {local }} \ldots \ldots \ldots . \ldots . \ldots 71$

4.2 Mechanical stability . . . . . . . . . . . . . . 74

4.2 .1 Contact numbers . . . . . . . . . . . . . . 74

4.2.2 Protocol dependency of contact numbers . . . . . . . 75

4.2.3 Global contact types and constraints . . . . . . . . 75

4.3 Characterization of geometric order . . . . . . . . . . 79

4.3.1 Translational order . . . . . . . . . . . . 79

4.3 .2 Orientational order . . . . . . . . . . . . 80

4.3.3 Densest arrangements . . . . . . . . . . . . . . 81

4.4 Local properties. . . . . . . . . . . . . . . . . . 85

4.4.1 Local packing fraction from Voronoi volumes . . . . . 86

4.4 .2 Local contact types . . . . . . . . . . . . . . 89

4.4.3 Local angle distributions . . . . . . . . . . . . . . 90

4.5 Comparison to DEM simulations . . . . . . . . . . . . 91

5 Packings of octahedra and spaghetti $\quad 95$

5.1 Octahedra . . . . . . . . . . . . . . . . . 95

5.2 Spaghetti packings . . . . . . . . . . . . . . . . 98

5.2 .1 Motivation ................... 98

5.2 .2 Preparation . . . . . . . . . . . . . . . . 98

5.2 .3 Particle detection . . . . . . . . . . . . . . . . . . . . . . . . . 99

5.2 .4 Analysis . . . . . . . . . . . . . . . . . 99 
6 Conclusion and Outlook 101

6.1 Tetrahedra packings are hyperstatic . . . . . . . . . . 101

6.2 Outlook . . . . . . . . . . . . . . . . . 102

$\begin{array}{ll}\text { A Image processing } & 105\end{array}$

A.1 Morphological image processing . . . . . . . . . . . . 105

A.2 Data structures . . . . . . . . . . . . . . 106

$\begin{array}{ll}\text { B Neudecker vs. Jaoshvili } & 107\end{array}$

$\begin{array}{ll}\text { C Variable listing } & 109\end{array}$

$\begin{array}{ll}\text { D Acknowledgements } & 119\end{array}$

$\begin{array}{ll}\text { E Publications } & 121\end{array}$ 
CONTENTS 


\section{Chapter 1}

\section{Introduction}

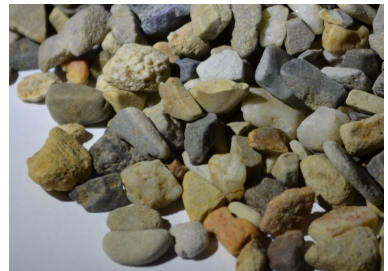

(a) Gravel

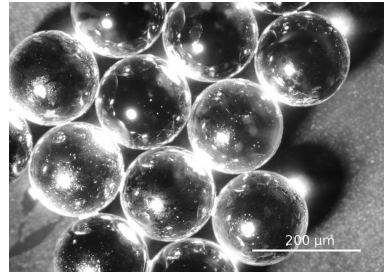

(b) Spherical beads

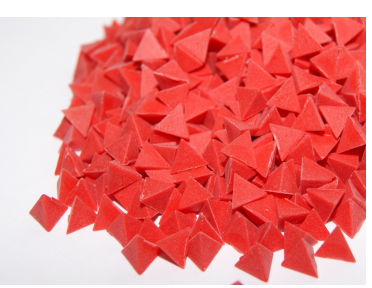

(c) Tetrahedra particles

Figure 1.1: Real granulate versus two experimental model granulates: (a) Gravel sample: cm-sized stones obtained from Naturbad Grone (b) Spherical beads (manufacturer MoSci), picture courtesy of Guido Schriever (c) Tetrahedra particles with $7 \mathrm{~mm}$ side length, the main subject of this study.

Granular materials are ubiquituous in nature, and utilized in many industrial procedures, for example in pharmaceutical production, or the handling of bulk commodities like grains or coal. The constituents of granular materials vary widely in their size and shape, ranging from fine sand with grain sizes of less than $1 \mathrm{~mm}$ to meter-sized boulders, or from spherical pepper to polyhedral salt grains. A key property of static granulates is the packing fraction ${ }^{1} \Phi$, which is the fraction of space occupied by grains within a defined volume. In many cases, high packing fractions are desirable, for example the economic transport of goods. In practice, empiric filling protocols are used [74]. However, optimization of these procedures requires a deeper understanding of how packing works. This can only be achieved by abstraction from the engineer's perspective, and more closely examining individual parameters, such as the link between particle shape and observed packing fractions. This question is not settled yet, although an abundance of polyhedral shapes has been investigated computationally [17]. Thus, the practical questions how much grain a barrel can hold, or how much coal fits

\footnotetext{
${ }^{1}$ Also termed volume fraction or packing density
} 
in a transport wagon are quite intricate. Moreover, the dynamic behaviour of granulates is not understood completely, leading e.g. to the recurring collapse of grain silos [33, 11].

There is still a mismatch between real granulates and modeling approaches, attributable to the "spherical cow" simplification: ${ }^{2}$ Fig. 1.1 illustrates the difference between a real gravel granulate, mono-sized spherical glass beads as typical grains-in-the-lab and the tetrahedral particles used in this work. In theoretical and numerical studies, particles are commonly approximated by frictionless spheres [45, 58]. Only in the past decade, granulates composed of non-spherical particles have been systematically investigated through experiments, like the packing structure of ellipsoidal M\&M candies $[18,19]$ or the mechanical response of various 3D-printed particle assemblies[4]. Considering polyhedral particles, experiments on tetrahedral dice have been limited to the analysis of packing fractions $[6,96]$, or the packing structure of a single sample [34].

The following sections present two different perspective on granulates: The first is the mechanical perspective, which tackles questions of mechanical stability in the context of the Jamming paradigm (Sec. 1.1). The second interprets a "grain" as a ideal geometric object and puts it in the context of mathematical packing problems (Sec. 1.3). In particular, the regular tetrahedron has interesting features both from the mechanical and the geometrical viewpoint. In Sec. 1.2, the analysis of local packing fractions is motivated briefly by the statistical mechanics approach to granular matter.

\subsection{Mechanical stability of granulates}

Grains are essentially non-deformable particles without cohesion, which can interact and transmit forces through mechanical contacts [32]. A pile of sand appears like a solid; it is mechanically stable in the presence of gravity, because the thermal energy is much smaller than the energy needed to lift a particle. ${ }^{3}$ When external agitation like tapping or vibration is applied, the resting grains are temporarily fluidized and settle to a different arrangement.

The mechanical stability of granulates is mainly determined by the average contact number $Z$, which counts the mechanically contacting neighbors per particle. First contact numbers of sphere packings were determined more

\footnotetext{
${ }^{2}$ The joke refers to the abstracted, simplified assumptions made in theoretical models and goes like this: The agricultural ministry wants to increase milk production and asks the best mathematicians and physicists of the country to come up with a practical solution. After a few days, they proudly present it: "We have found a solution, but it works only for a spherical cow in a vacuum."

${ }^{3}$ At room temperature of $T=300 \mathrm{~K}$, compare the thermal energy of $E_{t h}=k_{B} T \approx$ $10^{-21} \mathrm{~J}$ to the potential energy $E_{\text {pot }}$ needed to lift a grain with mass $1.3 \cdot 10^{-6} \mathrm{~kg}$ (spherical glass bead with diameter $1 \mathrm{~mm}$ and density $2500 \mathrm{~kg} / \mathrm{m}^{3}$ ), by only $0.1 \mathrm{~mm}$ : $E_{\text {pot }}=m g h \approx 10^{-9} \mathrm{~J} E_{t h}$.
} 
than 50 years ago in the pioneering work of Bernal [9]. A packing of steel balls in a container was flushed with paint, then drained, with the paint remaining and allowed to dry at the contacting spots. After disassembling the packing, the number of paint spots per particle was counted manually, and ascribed to the contact numbers of $Z=5.5$ and $Z=6.5$ are in line with current tomographic experiments [3]. More recent techniques involve local force measurements [46], which allow the non-intrusive distinction of real contacts to close neighbors. There is a subtle difference in these analysis techniques: Tomographic analysis, as employed in this work, does not provide contact forces, but instead the geometric contact number, which is motivated in Sec. 3.2 and evaluated in Sec. 4.2.

Experiments show that the internal distribution of contact forces in a granular pile varies widely, depending on the way the pile was prepared [5]. This feature is commonly observed in experimentally prepared granulates, and is rooted in their dissipative property: When grains are poured into a container, they lose their energy quickly by collisions, and settle due to gravity and inter-particle friction, before exploring all mechanically possible configurations. Even in the simple case of a ball settling into a groove, the distribution of normal and tangential forces at the contacts is indeterminate and depends on the movements just prior to settling [28]. The preparation protocol (or history) is therefore an important control parameter in granular experiments. In this work, the influence of different experimental preparation protocols (Sec. 2.2) on tetrahedra packings is analysed explicitly in Sec. 4.1.

The minimum number of contacts $Z$, which renders a granulate mechanically stable, can be determined via constraint counting: By the isostatic conjecture (ascribed to Maxwell), the number of contacts must be equal to twice the degrees of freedom (DOF) per particle. Frictionless spheres with 3 translational DOF therefore have a isostatic contact number of $Z_{J}=Z_{i s o}=2 \cdot 3=6$. Generally, a packing is termed hyperstatic, if $Z>Z_{\text {iso }}$ and hypostatic if $Z<Z_{\text {iso }}$.

\subsubsection{Jamming of spheres}

\section{Frictionless case}

The Jamming paradigm provides a unifying framework for the static and dynamic behaviour of disordered systems, including glasses, emulsions, foams and granular matter. All these particulate systems can undergo a transition from a unjammed or fluid-like regime with lower density, to a jammed, solid-like state with higher density. This is termed the Jamming transition or $J$; properties like the packing fraction $\Phi$ at Jamming are denoted by the subscript $\Phi_{J}$. In the model of frictionless sphere packings, the Jamming transition occurs at the well-defined density $\Phi_{J}=0.639 \pm 0.001$ [58], which 
coincides with the so-called random close packing limit (RCP). Recent results suggest that this limit is actually composed of multiple transitions, pushing the RCP limit to $\Phi=0.65[7]$. Above RCP, packings start to crystallize locally in fcc (face-centered-cubic) patches, which finally lead to the maximum packing fraction of $\Phi \approx 0.74$.

Numerical simulations have revealed that mechanical properties (e.g. bulk modulus or elastic energy) scale with the density difference to the Jamming point as $\sim\left(\Phi-\Phi_{J}\right)^{\beta}$. Here, $\beta$ depends on the type of employed inter-particle potential, and ranges from 2 to $5 / 2$ [58]. In particular, for the so-called "excess" contact number $Z-Z_{J}$, where is the $Z_{J}$ contact number at point $J$, a universal square-root dependence in $\Phi-\Phi-J$ is found:

$$
Z-Z_{J} \sim\left(\Phi-\Phi_{J}\right)^{1 / 2}
$$

Here, the excess contacts and the higher density $\Phi>\Phi_{J}$ are reached by compression of the particles.

Note that $Z$ is not necessarily positively correlated with $\Phi$ : For example, Jiao et. al.[36] numerically constructed packings of frictionless spheres with densities as low as 0.49 ("Tunneled Crystals"). Particularly, for a subset of packings between $\Phi=0.602$ and $\Phi=0.663$, the contact number even decreases from 6.3 to 6.1 .

A note of caution here regarding the "soft" potentials employed in simulation: Real granulates are composed of hard particles, which can not interpenetrate each other, but deform slightly under compression. For ease of computation, model particles are usually treated as non-deformable, but "soft", which means that they can overlap each other, and follow a repulsive contact law, e.g. an elastic Hertzian law [58].

\section{Frictional case}

When friction is introduced, isostatic contact numbers can take a range of values from $Z_{\text {iso }}=4$ (for infinite friction) to $Z_{\text {iso }}=6$ for the frictionless case. In practice, the observed contact number at Jamming depends on the preparation history and the particles' friction coefficient $\mu$, so that isostaticity and Jamming do not coincide anymore [77, 29]. With friction, less contacts are needed for mechanical stability, because each point contact imposes three constraints: one normal force (as in the frictionless case), and two additional tangential forces due to friction. Numerics and experiments [8, 3, 77] confirm that contact numbers of frictional spheres are observed within these bounds. Accordingly, the range of mechanically stable packings extends to looser packings (i.e. lower range of $\Phi$ ) in frictional sphere packings: The lower bound of $\Phi$, termed random loose packing (RLP), is presumably at $\Phi_{R L P}=0.550 \pm 0.001$, as investigated by fluidized bed experiments [35].

A comprehensive survey on numerical studies on Jamming of soft particles, which covers also features of frictional and non-spherical particles, is 
given by M. van Hecke [90].

\subsubsection{Jamming of tetrahedra}

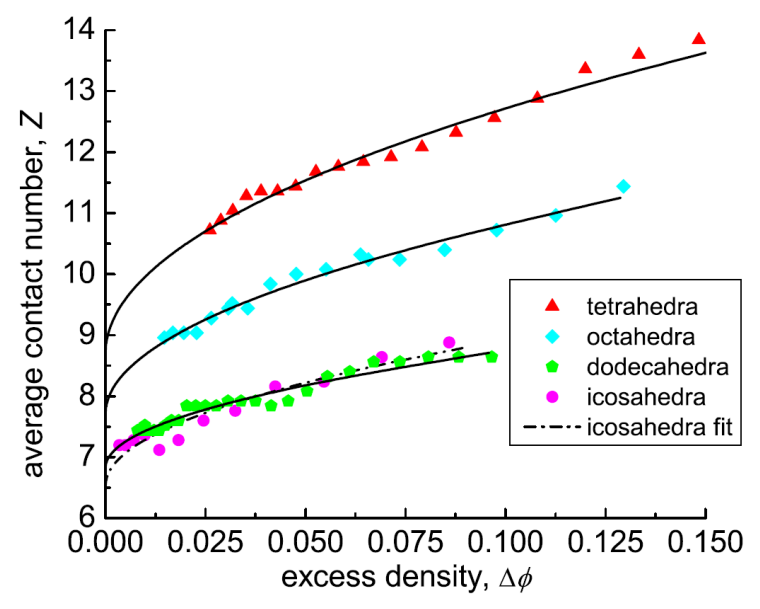

Figure 1.2: Scaling of contact number $Z$ with distance to Jamming by the excess density $\Delta \phi=\Phi-\Phi_{J}$. Jamming thresholds of Platonic bodies are obtained by extrapolation of square-root fits (Eq. 1.1) to $\Delta \phi=0$. Taken from Ref. [80]

Non-spherical particles like tetrahedra [34, 52], reach higher packing densities than spheres, both in disordered and ordered configurations. However, the contact number is insufficient to characterize mechanical stability or distance to isostaticity as in Eq. 1.1. Instead, the different contact geometries (face-face, edge-face, vertex-face or edge-edge configurations) need to be considered: they are associated with a different number of forces which affect the total number of mechanical constraints. The solution to this problem is presented in Sec. 3.3: The contact number $Z$ is replaced by a generalized constraint number $C$, which accounts for the different contact geometries. In this way, one can still define a meaningful distance to isostaticity. Sec. 4.2.3 presents and discusses the experimentally observed relationship between constraints and packing fraction in the context of Jamming.

Smith et al. showed in numerical simulation of soft frictionless Platonic solids, that the contact number $Z$ still follows a square-root scaling when $\Phi$ exceeds the Jamming threshold (Fig. 1.2). There is however a subtlety involved in these simulations: Above the jamming point, particles are compressed, and the soft potential allows for substantial overlaps, hence the packing fractions and contact numbers are likely to differ from experimental results. Additionally, when approaching the Jamming threshold from above, stable packings are not found anymore due to numerical instabilities, which explains the need for an extrapolation to $\Phi_{J}$ and $Z_{J}$ in Fig. 1.2.

Again, the monotonic increase of $Z$ with $\Phi$ is not set in stone; a coun- 
terexample is the lattice packing of tetrahedra, which has the lowest density of $\Phi \approx .367$, but the highest contact number of $Z=14$. Nevertheless, the lattice packing is supposedly unstable in practice, because only point contacts are present. Ref. [15] contains a sketch of the actual lattice packing, Fig. 1.3b illustrates only a regular arrangement of tetrahedra.

\subsection{Statistical mechanics of granular matter}

A more fundamental question is: Can we find a thermodynamic description of granular matter in the framework of statistical mechanics [14]? Such a theory would permit to describe mechanically stable granulates by only a few parameters. Results from numerical simulation support this approach [47], but the validity of such a statistical theory is still under debate.

\subsubsection{Voronoi volumes}

In Edwards' formulation [21] of the statistical theory, the particles' "effective volume" plays a crucial role. ${ }^{4}$ The effective volume is the region of space, which is closer to a given particle than to any other, and hence is uniquely attributed to this particle. The Voronoi decomposition achieves this by tesselating space into Voronoi regions (cells), and can be generalized for arbitrary particle shapes as the "navigation map" or "set Voronoi diagram" [71]. The technical implementation is outlined in Sec. 3.4, and all results considering local packing fractions are based on the Voronoi volumes (Sec. 4.4).

\subsection{Packing problems}

We all are familiar with packing problems: cramming bought groceries into a bag, packing suitcases tightly into a car trunk, or the economically relevant question of maximizing transportation of bulk goods in containers. In all granular applications, the particle shape and the container (boundary effects) play a role for efficient storage.

\subsubsection{Tetrahedral packaging}

In the 1940s, when milk was sold in heavy glass bottles, the Swedish businessman Ruben Rausing invented and patented a tetrahedron shaped carton for packaging liquids under the name "Tetrapak" ${ }^{\circledR}$. The company Tetrapak AB was established in 1951, but the commercial break-through was only reached with the "Tetra Brik" (1963), which is the commonly known

\footnotetext{
${ }^{4}$ Edwards' chose this wording to differentiate from the "free volume" which had been commonly used in liquid and glass theory.
} 
milk package today (a parallelepiped). The original tetrahedral shape is aestethically appealing and gives rise to interesting packing structures, as advertising pictures from the company show (Fig. 1.3).

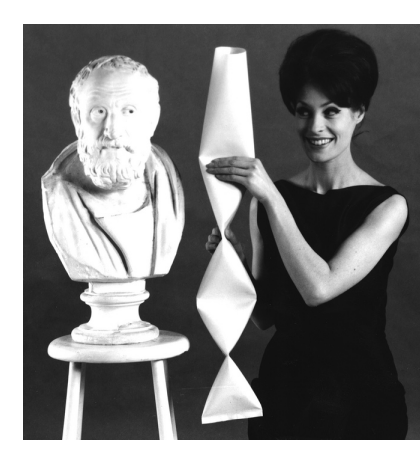

(a)

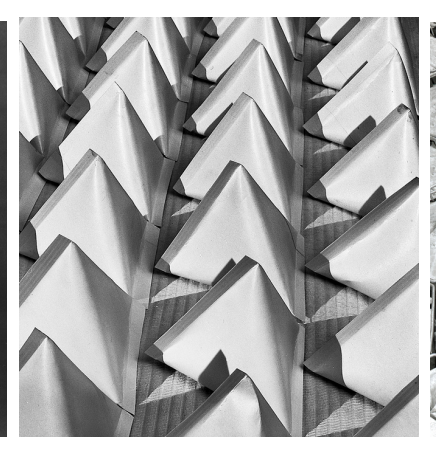

(b)

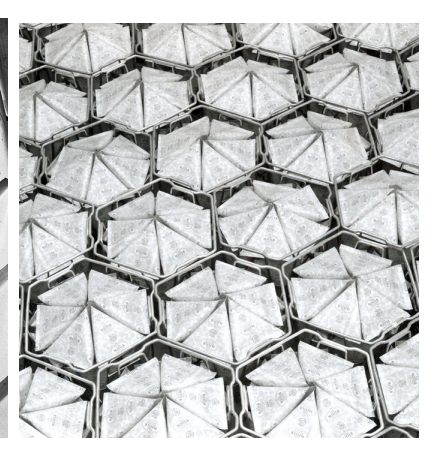

(c)

Figure 1.3: Tetrapaks ${ }^{\circledR}$ in different configurations. (a) Bust of Plato, and manual demonstration of the production process by sealing a paper tube in alternatingly orthogonal directions (b) Production line, resembling a lattice packing (c) Storage in hexagonal baskets. All pictures by the company under creative commons licence [84].

\subsubsection{From Hilbert to the New York Times: Pursuing the perfect packing}

The relationship between particle shape and optimal packing density is part of Hilbert's famous list of problems: In 1900, the mathematician David Hilbert held a famous speech in Paris, where he listed 23 unsolved mathematical problems which he considered the most important for future progress. Among others, the 18th problem concerns the packing of identical objects:

\section{Hilbert's 18th problem, Paris, 1900}

... Ich weise auf die hiermit im Zusammenhang stehende, für die Zahlentheorie wichtige und vielleicht auch der Physik und Chemie einmal Nutzen bringende Frage hin, wie man unendlich viele Körper von der gleichen vorgeschriebenen Gestalt, etwa Kugeln mit gegebenem Radius oder reguläre Tetraeder mit gegebener Kante (bez. in vorgeschriebener Stellung) im Raume am dichtesten einbetten, d.h. so lagern kann, daß das Verhältnis des erfüllten Raumes zum nichterfüllten Raume möglichst groß ausfällt. [30]

In short: What is the closest packing of identical objects in space explicitly mentioning spheres or regular tetrahedra? ${ }^{5}$ More than 100 years

\footnotetext{
${ }^{5}$ In a sidenote, Hilbert also raises the question of the densest lattice packing of tetrahedra, an arrangement where all tetrahedra have the same orientation.
} 
later, K. Chang from the New York Times used a slighty more popular wording:

\section{"Scientists Take On Tetrahedral Packing Puzzle" NYT January 4, 2010}

Now, in the past year, a flurry of academic activity is suddenly zooming in on an answer to a problem akin to wondering how many people can fit into a Volkswagen Beetle or a phone booth. Except here mathematicians have been thinking not about the packing of people, but of geometric solids known as tetrahedrons.

For spheres, the densest packing resembles the stacked arrangement of oranges at a grocery store, corresponding to the face-centered-cubic or hexagonal-close-packing with $0.740 \ldots$ volume fraction. Kepler conjectured this in 1611 to be densest packing, but only in 1998, Hales succeeded with a computer-assisted proof [27]. The puzzle of perfect packings of tetrahedra is not yet settled conclusively, and its history has been full of wrong assumptions. In ancient times, Aristotle mistakenly assumed that tetrahedra would pack perfectly, i.e. there existed a space-filling structure, a misconception which persists until today.

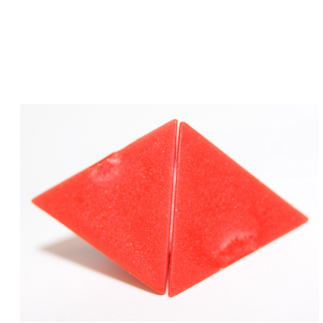

(a) Dimer

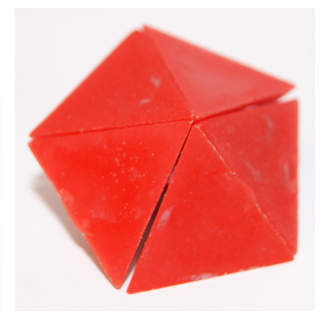

(b) Pentamer

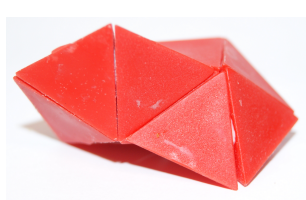

(c) Nonamer

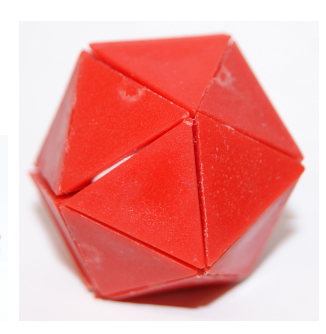

(d) Icosahedron

Figure 1.4: Suggested building blocks of dense tetrahedra packings (models stuck together with putty). (a) Dimer: Pair of tetrahedra aligned face-face (b) Pentamer, also termed "wagon-wheel" [86, 85] or "pentagonal dipyramid" [26]: 5 tetrahedra arranged around a common edge (c) Nonamer (9 tetrahedra), composed of two orthogonally interpenetrating pentamers [13] (d) Icosahedral structure with 20 tetrahedra [15]

The manual construction of clusters with putty is a playful yet effective starting point to the packing problem of tetrahedra. Fig. 1.4 illustrates different possible "building blocks" for dense packings which are referred to later. Note that these clusters are not even locally space-filling: The pentamer is not a closed ring, but has a gap of $7.36^{\circ}$ between the last and the first tetrahedron, and the icosahedral cluster has a gap of 1.54 steradians. The putty distributes these gaps between the particles. Only a dimer configuration (two tetrahedra aligned face-to-face) is locally spacefilling, because it occupies $100 \%$ of its convex hull. 


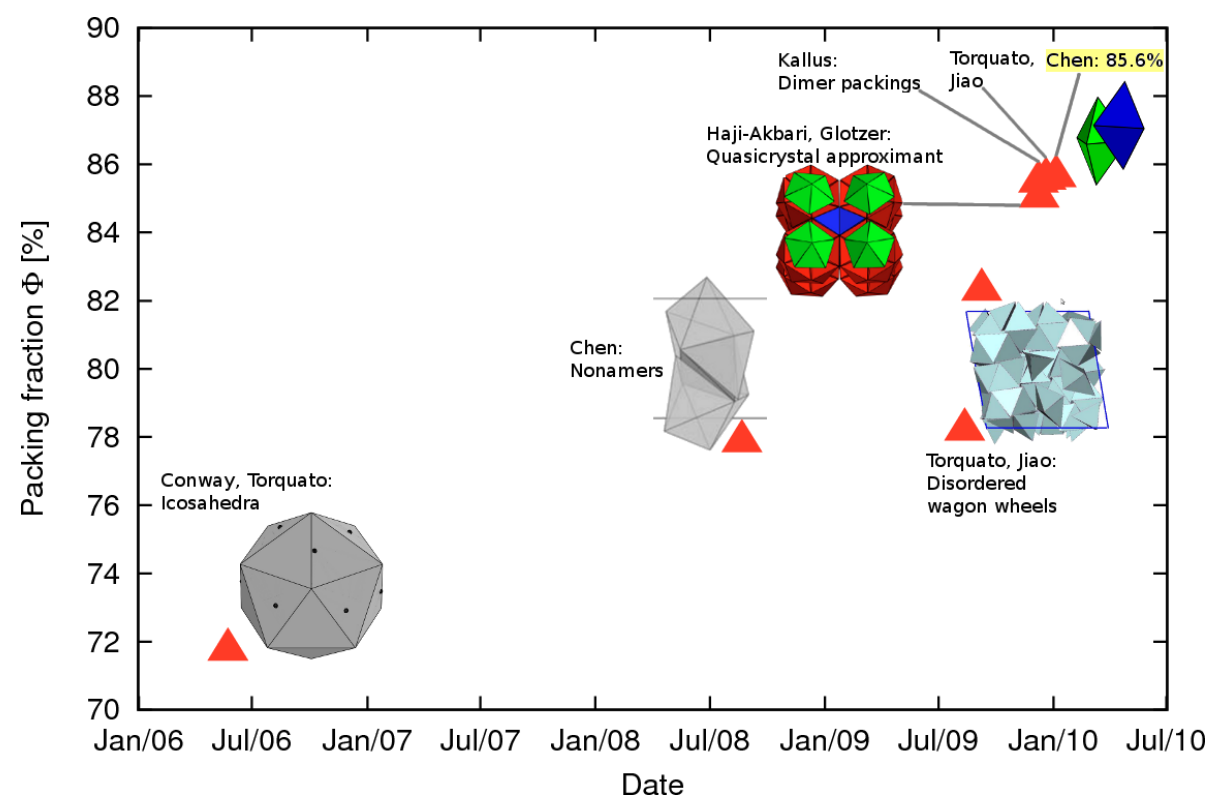

Figure 1.5: The quest for the densest packing of tetrahedra

The pursuit for the perfect packing of tetrahedra was picked up again in 2006 by Conway and Torquato, who presented a packing structure filling nearly $72 \%$ of space [15], based on an icosahedral arrangement as in Fig. 1.4d. Fig. 1.5 illustrates that this was the starting shot for a 4-year long quest for the optimal packing, with accelerating pace until the presumable upper bound in 2010. They concluded with a bold prediction:

\section{Conway and Torquato:}

"However, it appears unlikely that the density of the optimal packing of regular tetrahedra will exceed the optimal density of $74.048 \ldots$ for congruent spheres. (...) The regular tetrahedron might even be the convex body having the smallest possible packing density." [15]

This conjecture was soon disproven by E. Chen[13], who analytically constructed a dense packing of regular tetrahedra with $\phi \approx .7786$, composed of a nonamer unit cell as in Fig. 1.4c. Later, Torquato and Jiao performed Monte Carlo (MC) simulations, which lead to a packing fraction of $\phi=$ .782 [86]; a different preparation procedure yielded even $\phi=.8226$ [85]. They termed these packings "disordered wagon-wheels", a configuration of percolated pentamers or "wagon-wheels" (see Fig. 1.4b).

This was soon surpassed by Haji-Akbari et al., who observed a quasicrystalline arrangement in Monte-Carlo simulations, and by compressing an approximant of that structure, they found a density of $\Phi=0.8503$. 
Kallus[39] finally proposed a family of double-dimer configurations (see upper right corner in Fig. 1.5), composed of 4 tetrahedra in a unit cell. Small changes in these configurations led to a rapid succession towards the supposed upper limit: From $\Phi=.8547[39]$ to $\Phi=.8555$ [87] and finally to $\Phi_{\max }=.8563[12]$. Maybe this discovery paves the way for a revival of the original Tetrapak ${ }^{\circledR}$ ?

In short, a rich variety of dense tetrahedra packings, ranging from the double-dimer up to the quasi-crystal comprising 82 particles in a unit cell, have been revealed by analytical construction or numerical simulation. But these approaches do not account for mechanical forces, leaving a crucial question open:

\section{Which packing fractions and geometric structures are accessible and mechanically stable in experiments?}

The answer is not trivial - for example, one might intuitively suspect that the "simple" dimer packing is favoured over the "complicated" quasicrystal. However, the dimer structure is thermodynamically unstable at all practical pressures, as a proposed phase diagram of hard tetrahedra reveals (isochoric and isobaric MC simulations by Haji-Akbari et al.[25]). By construction, all of these packings have repeating unit cells and are therefore highly ordered. In the present experiments, different metrics for characterization of the disordered packing structure are employed (Sec. 4.3). In analogy to a local crystallization in sphere packings above the RCP limit, tetrahedra packings might exhibit ordered patches of (yet) unknown structure. This leads to another question of practical relevance:

\section{Which metrics characterize order in disordered tetrahe- dra packings?}

\subsubsection{Characterization of order}

Bond-order parameters have been successfully employed to characterize orientational order in sphere packings [3]. The notion of "orientational" order refers here to the angular distribution of vectors connecting a sphere to its surrounding neighbors. Application of this order metric to non-spherical particle packings is restricted by two limitations: First, the measured parameters (particularly $Q^{4}$ for four-fold and $Q^{6}$ for six-fold symmetries) are always characterized relative to signatures from known crystal structures, like the hcp (hexagonal close packing) arrangement. As we have seen, tetrahedra do not possess a single crystal structure, but a variety of dense packings exists instead. The second limitation concerns the shape difference: The bond-order parameters are computed only from the centroid positions of spheres, which are invariant to rigid-body rotations. In contrast, the orientational alignment is a key feature of non-spherical particles. 
Therefore, the translational and orientational order correlations, which successfully characterize the maximum random jammed packing of spheres [88], are of limited use for jammed tetrahedra packings. Significant correlations extending further than the nearest neighbor are only found for highly ordered structures like the wagon-wheels at $\Phi=.782[86]$ or the quasicrystal at $\Phi>.832[26]$, as a recent comparison study points out[76]. This effect is probably ascribed to the radial averaging in $g(r)$ and $F(r)$, which does not account for the angular neighbor arrangements of non-spherical particles.

A systematic study of jammed packings of platonic bodies revealed that increasing sphericity leads to an increase of translational order, but a decrease of orientational order [37]. The $g(r)$ of an icosahedron packing resembles a dense, disordered sphere packing $(\Psi=1)$. On the other hand, correlations in $C(r)$ (resp. $F(r)$ ) extend furthest for tetrahedra, whereas orientational correlations of the icosahedron decay immediately. This trend is expected: Approaching the sphere limit with an infinite number of facets, $C(r)$ becomes meaningless. The relation between sphericity and packing fraction is illustrated in Tab. 1.3.

\subsubsection{Perfect vs. physical tetrahedra}

Only few experiments have been done on packings of tetrahedral particles up to now: Baker and Kudrolli[6] performed fluidized bed experiments on ceramic tetrahedra with a friction coefficient $\mu \approx 0.480$, resulting in a lowest packing fraction of $\Phi_{\text {min }}=0.48 \pm 0.02^{6}$. They also found that plastic tetrahedra with lower friction $(\mu \approx .375)$ reached $\Phi_{\min }=0.51 \pm 0.01$, and on the upper end of the range, $\Phi=0.64$ was reported.. For packings of tetrahedral dice, densities up to $\phi=0.73$ were reached (single dataset, tomographically measured [34]), and supported recently by Zhao et al. [96] with $\phi=0.715$. Both authors claim even higher values of $\phi=0.76 \pm 0.02$ resp. $\phi=0.749 \pm 0.004$, if the densities are extrapolated to infinitely large containers. The physical tetrahedra are not ideal geometric particles, regarding roundness of edges and corners, flatness of faces and frictional properties. How comparable are the packings of physical tetrahedra to the ones constructed by numerics or analytically? One possibility is to use more realistic models of the particle shapes in simulations. In table 1.1, studies on packings of "imperfect", physical tetrahedra models are compiled.

This involves the construction of the densest unit cell of tetrahedral "puffs" [38], Monte Carlo simulations of truncated tetrahedra[17], and DEM simulations of spherotetrahedra[96, 62, 61]. The different particle shapes and the obtained range of densities are illustrated in Tab. 1.1. In order to draw meaningful analogies between numerics and experiment - apart from the geometric shape - the technical details of the simulations (ensemble,

\footnotetext{
${ }^{6}$ The large error is due to the determination of $\Phi$ via the packing height.
} 


\begin{tabular}{|c|c|c|c|}
\hline \multirow[t]{4}{*}{ Shape } & $\begin{array}{l}\text { Description, } \\
\text { Reference }\end{array}$ & $\begin{array}{l}\text { Parameter, } \\
\text { Range }\end{array}$ & Obtained $\Phi$ \\
\hline & $\begin{array}{l}\text { Tetrahedral puff, } \\
\text { numerical search[38] }\end{array}$ & $\begin{array}{l}\text { Asphericity } \\
1 \text { (sphere) } \\
3 \text { (ideal tet.) }\end{array}$ & {$[0.74 \ldots 0.856]$} \\
\hline & $\begin{array}{l}\text { Spherotetrahedron, } \\
\text { DEM simulation }[61,96]\end{array}$ & $\begin{array}{l}\text { Sharpness } \\
0 \text { (sphere) } \\
\infty \text { (ideal tet.) }\end{array}$ & {$[0.59 \ldots 0.72]$} \\
\hline & $\begin{array}{l}\text { Truncated tetrahedron, } \\
\text { MC simulation }[17]\end{array}$ & $\begin{array}{l}\text { Truncation } \\
0 \text { (ideal tet.) } \\
1 \text { (octahedron). }\end{array}$ & {$[0.856 \ldots 1.0]$} \\
\hline
\end{tabular}

Table 1.1: Overview of "physical" tetrahedra models used in numerical simulation. Packing fractions vary along the specific shape parameter. Tetrahedral puffs: Lower bound of $\Phi$ corresponds to the crystalline sphere packing, upper limit concides with the dense dimer packing[12]; Spherotetrahedra: Lower range of $\phi$ due to friction, which is an additional control parameter; Truncated tetrahedra: Truncation of $1 / 3$ allows space-filling structure ( $\beta$-tin crystal[17])

potential, pressure...) are crucial. The tetrahedral puffs model nicely the transition from a sphere $\left(\phi_{\max }=0.74\right)$ to a tetrahedron $\left(\phi_{\max }=0.856\right)[38]$. The effect of decreasing packing fraction when the sphericity increases has been confirmed recently [96]. However, the packings of puffs are constructed numerically without considering mechanical stability, similarly to the frictionless truncated tetrahedra from MC simulations [17]. The DEM simulations of spherotetrahedra [61] with appropriately defined sharpness parameter resemble the experiments (both with dice and our particles) best: They explicitly incorporate friction, gravity and an experimentally motivated preparation technique (pouring+shaking). In Sec. 4.5, packing fractions and contact numbers of the DEM model of spherotetrahedra are compared to our experiments.

\subsection{Geometry of platonic bodies}

This section summarizes the relevant geometric properties of the regular tetrahedron and the octahedron, the objects which are investigated in this study. These polyhedra are two of the five platonic bodies, comprising 
tetrahedron (4 faces), cube (6 faces), octahedron (8), dodecahedron (12) and icosahedron (20). They share the follwing properties: All platonic solids are composed of congruent regular polygons, and their vertices lie on a sphere, which is the circumsphere with radius $R_{\text {out }}$ (= centroid-vertex distance). Accordingly, the insphere with radius $R_{i n}$ touches all faces, and corresponds to the minimal centroid-face distance. Fig. 1.6 illustrates the geometry of the regular tetrahedron with inradius and circumradius. Selected geometric properties of tetrahedron and octahedron are shown in Table 1.2, where the parameter $a$ denotes the side length (edge length).

\begin{tabular}{|cccc|}
\hline Particle & Inradius $R_{i}$ & Circumradius $R_{o}$ & Volume \\
\hline Tetrahedron & $\frac{1}{2 \sqrt{6}} a$ & $\frac{3}{2 \sqrt{6}} a=3 R_{i}$ & $V_{\text {tet }}=\frac{\sqrt{2}}{12} a^{3}$ \\
Octahedron & $\frac{1}{\sqrt{6}} a$ & $\frac{a}{\sqrt{2}}=\sqrt{3} R_{i}$ & $V_{\text {oct }}=\frac{2}{3} a^{3}$ \\
\hline
\end{tabular}

Table 1.2: Selected geometric properties of regular tetrahedron and octahedron

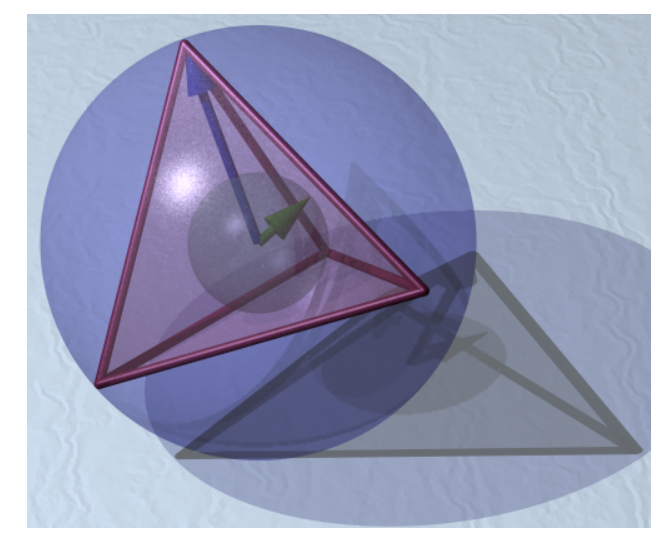

Figure 1.6: Regular tetrahedron with circumsphere of radius $R_{o}=3 R_{i}$ (blue arrow) and inscribed sphere of radius $R_{i}$ (green arrow).

A general distinction criterion between spheres and non-spherical particles is the sphericity $\Psi$, which is derived from the ratio of sphere volume $V_{s}=\frac{4}{3} \pi r^{3}$ to surface $A_{s}=4 \pi r^{2}$. For a arbitrary object with surface $A$ and volume $V$, sphericity is computed as:

$$
\Psi=\frac{\pi^{\frac{1}{3}}(6 V)^{\frac{2}{3}}}{A} .
$$

The sphericities of tetrahedron, octahedron and icosahedron compared to the sphere $(\Psi=1)$, with their maximum packing density are listed in Tab. 1.3. Ulam's conjecture ${ }^{7}$ states that the sphere should have the lowest

\footnotetext{
${ }^{7}$ Appeared as a footnote in Martin Gardner's "Colossal Book of Mathematics" (Norton,
} 


\begin{tabular}{llll}
\hline Particle & Sphericity $\Psi$ & $\Phi_{J}$ & $\Phi_{\max }$ \\
\hline \hline Tetrahedra & 0.67 & $0.61^{*}$ & $0.856^{\dagger}$ \\
Octahedra & 0.85 & $0.68^{*}$ & $0.947^{\ddagger}$ \\
Icosahedron & 0.94 & $0.73^{*}$ & $0.836^{\ddagger}$ \\
Sphere & 1.0 & $0.639^{\circ}$ & 0.740 \\
\hline
\end{tabular}

Table 1.3: Overview of selected platonic bodies with their asphericity, Jamming threshold, and densest known geometric packings. ${ }^{*}$ from Ref. [80], ${ }^{\dagger}$ from Ref. [12], $\ddagger$ from Ref. [85], ${ }^{\circ}$ from Ref. [58]

packing density of all convex bodies, which holds also for the platonic bodies. However, maximum packing density $\Phi_{\max }$ does not decrease monotonely with sphericity, as Tab. 1.3 points out. 


\section{Chapter 2}

\section{Experimental setup}

The setup description covers first the different techniques for preparation of tetrahedra packings, and then outlines the stages of image acquistion by X-ray tomography.

\subsection{Particle characterization}

The tetrahedra particles with a side length of $7.0 \mathrm{~mm}$ are produced by injection moulding of polypropylene by Neu \& Biermann GmbH, and have a density of $0.96 \mathrm{~g} / \mathrm{cm}^{3}$. The close-up photograph in Fig. $2.1 \mathrm{~b}$ shows that the particles' surface is not smooth, and therefore substantial friction is expected. The friction coefficient (inter-particle friction) is determined by a tilting plane setup: A set of tetrahedra is fixed with one corner onto the tilting plane with putty, the others are placed face-down on top. From the distribution of tilting angles $\gamma$, when the particles slide past each other, the friction coefficient $\mu=\tan \gamma=0.87 \pm 0.03$ is computed.

The deviations from the ideal regular tetrahedron are characterized by inspecting cuts of mold imprints in silicone rubber (Smooth-On) with a optical microscope ${ }^{1}$. The typical corner and edge radii of the polypropylene particles was measured to be in the range of $150 \pm 50 \mu \mathrm{m}$, which is approximately $2-3 \%$ of the side length. Fig. 2.1a depicts a comparison to the tetrahedral dice used in the study by Jaoshvili et al. [34]. These dice have a side length of $20 \mathrm{~mm}$ and a corner radius of $1.5 \mathrm{~mm}$, which amounts to a relative curvature of $7-8 \%$.

\subsection{Packing preparation - shaken, not stirred}

A commonly used method to explore the configurational space of granulates is a constant input of energy, which brings the granulate from the static

\footnotetext{
${ }^{1}$ This imprinting method is also used to create high-quality replicas of fossiles.
} 


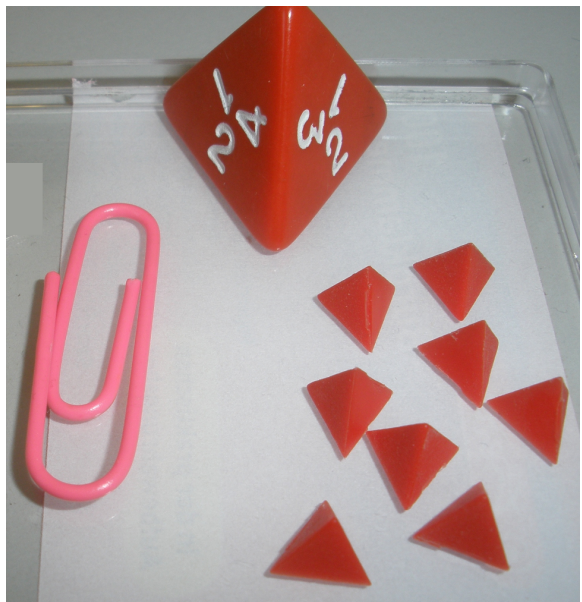

(a)

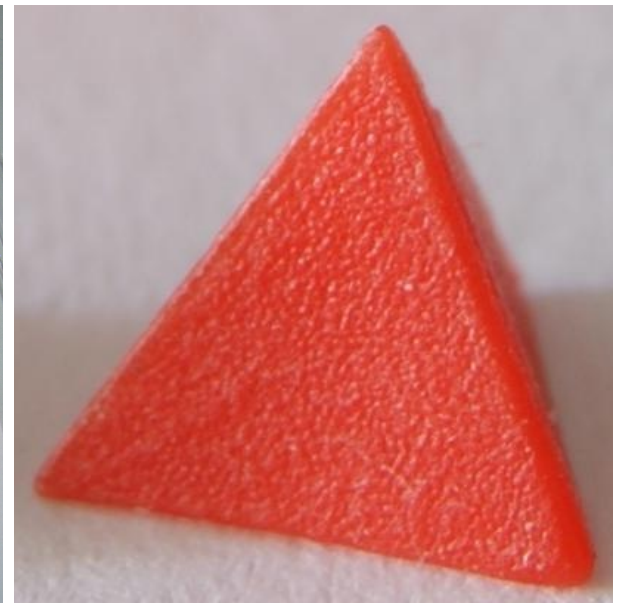

(b)

Figure 2.1: Photographs of tetrahedral particles. (a) Front: our particles, back: tetrahedral dice [34] with side length $20 \mathrm{~mm}$. (b) Close-up photograph of tetrahedral particle (side length $7 \mathrm{~mm}$ ) used in this study

to fluidized state. This can be achieved by mechanical vibration $[68,66]$ or fluidization by water $[73,35]$. The present shaker setup applies vertical pulses or continuous vibration to a cylinder which holds the granulate sample. Fig. 2.2 shows a scheme of the complete setup, comprising a control unit (PC, signal generator and amplifier), the shaker, and measurement devices for acceleration and height of the packing. The different parts are shortly explained in the following.

\subsubsection{Electromagnetic vibration exciter (Shaker)}

The shaker model (LDS V555) is a electromagnetic exciter with the same working principle as a loudspeaker: According to Maxwell's equations, a varying current $\dot{I}$ induces a magnetic flux $B$. Thus, a sinusoidally changing input signal $I(t)$ induces a periodic variation of $B$, which is employed to transduce a magnetic force to the exciter table, where the vibration sample is mounted. Fig. 2.3 shows a sketch of the internal design.

The control parameters are amplitude and frequency, and the signal output can be chosen as continuous vibration or as a train of pulses. The transduced acceleration is measured at the exciter table (3-axis acceleration sensor: Kistler 8763B) and reported in terms of the dimensionless shaking intensity $\Gamma=A \omega^{2} / g$ with peak displacement $A[\mathrm{~m}]$, angular frequency $\omega=$ $2 \pi f$, gravitational acceleration $g=9.81 \mathrm{~ms}^{-2}$ and frequency $f[\mathrm{~Hz}]$. The above expression for the shaking intensity $\Gamma$ is derived by a simple harmonic ansatz: 


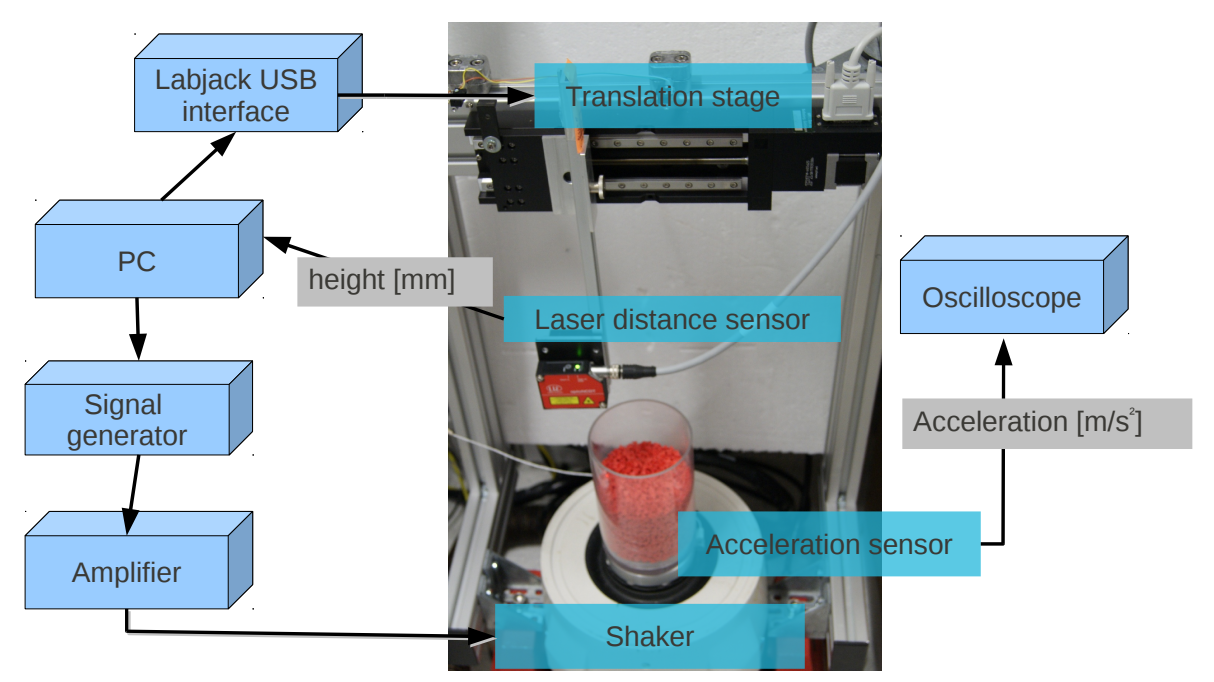

Figure 2.2: Experimental setup for shaker control and measurement: The signal generator is controlled via PC, and passes its signal through the amplifier to the shaker. Acceleration is measured directly at the exciter table and monitored on the osczilloscope. When the sample is at rest (before/after shaking), the laser distance sensor performs a line scan of the surface, driven by the translation stage.

$$
h(t)=A \sin (\omega t) \quad \Rightarrow \quad h^{\prime \prime}(t)=-\omega^{2} A \sin (\omega t) .
$$

The peak acceleration $a_{\text {peak }}=\max \left\{h^{\prime \prime}(t)\right\}_{t}$ is reached if $\sin (\omega t)=1$. After normalization by $g$, the above expression follows:

$$
\Gamma:=\frac{\left|a_{\text {peak }}\right|}{g}=\frac{A \omega^{2}}{g} .
$$

\subsubsection{Laser height sensor}

The laser distance sensor (ILD1302 by MicroEpsilon, Ortenburg) works by the triangulation principle: The device projects a laser spot onto the object surface and records the diffuse reflection simultaneously with a CCD line. If the object distance varies, the projection of the spot on the CCD is shifted, which allows computation of the distance by trigonometric relationships. The quality of the obtained data is influenced by the surface properties of the object. For example, specular reflections on metallic, shiny surfaces hamper the correct measurement; the ideal surface is flat, white, opaque, and diffusely reflecting. If the laser spot is occluded due to the presence 


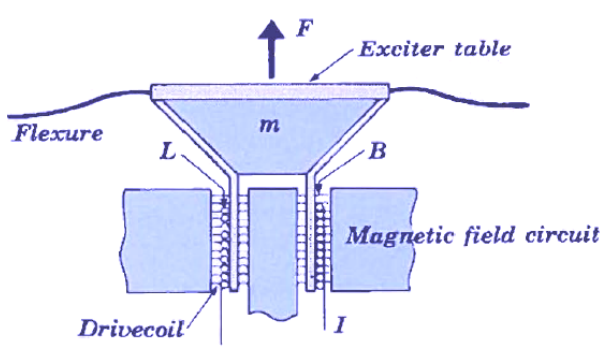

Figure 2.3: Working principle of a electromagnetic exciter (Brüel \& Kjaer handbook): The transduced force $F$ on the exciter table is proportional to the magnetic flux $B$, the coil length $L$ and the input current $I$.

of large height differences (trenches or protusions), no distance data can be acquired. The present model has a distance range of $100-200 \mathrm{~mm}$ with a resolution of $50 \mu \mathrm{m}$. Exemplary height profiles of the initial sample and after appyling 1600 taps are depicted in Fig. 2.4a. The standard deviation of the height profile decreases from the loose pile $\left(5.1 \mathrm{~mm}^{2}\right)$ to the compactified surface $\left(2.6 \mathrm{~mm}^{2}\right)$. When the absolut distance to the container bottom and the volume occupied by grain particles are known, the height measurement is a useful estimator of the packing fraction. Fig. 2.4b shows how the approximate $\Phi_{\text {height }}$ from the height measurement is calibrated with accurate packing fractions $\Phi_{\text {tomo }}$ from tomographic imaging.

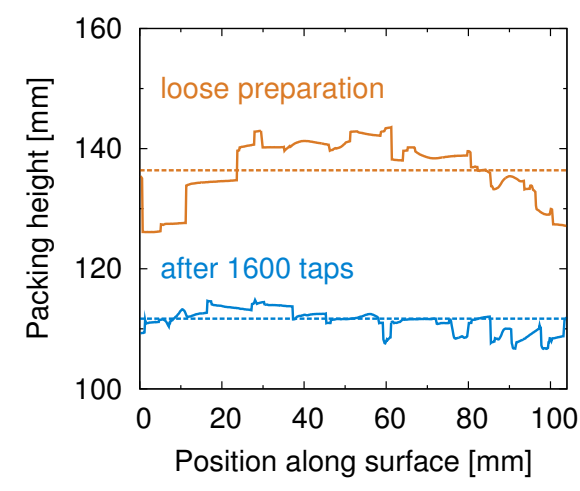

(a)

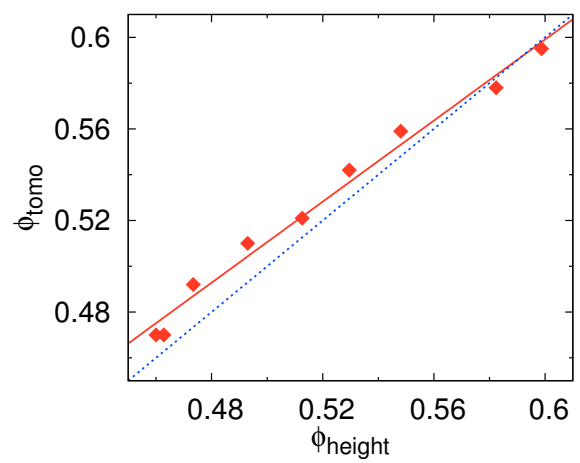

(b)

Figure 2.4: (a) Surface profile of packing as recorded by laser distance sensor for loose packing (red) and after applying 1600 taps at $\Gamma=2$. Dotted lines are average heights used for calibration. (b) Calibration of packing fraction measurement: The accurate $\Phi_{\text {tomo }}$ from tomographic reconstruction is proportional to the approximate $\Phi_{\text {height }}$ from height measurement (red line: linear fit, blue dotted line: ideal behaviour of $\Phi_{\text {tomo }}=\Phi_{\text {height }}$ for comparison) 


\subsubsection{Preparation protocols}

Four different preparation protocols are used to explore a wide range of packing fractions and investigate a possible protocol dependency of the packing structure. Fig. 2.5 gives an overview of the different protocols, namely Loose, TAP, RAMP and VIB and the corresponding range of $\Phi$.

\begin{tabular}{|l|l|l|}
\hline Preparation & Description & $\begin{array}{l}\text { Accessible packing } \\
\text { fractions }\end{array}$ \\
\hline Loose & $\begin{array}{l}\text { Gentle deposition of particles into a hollow } \\
\text { cylinder, which is slowly removed upwards }\end{array}$ & 0.47 \\
\hline TAP (default) & Sine pulses at $2 \mathrm{G}$ acceleration, 1...100K taps & $0.47-0.60$ \\
\hline TAP (high $\Gamma)$ & $\begin{array}{l}\text { Strong taps }(\Gamma=3 \ldots 7), 10 \mathrm{~K} \text { taps applied } \\
\text { RAMP }(\Gamma=5)\end{array}$ & $0.54-0.59$ \\
\hline RAMP $(\Gamma=025)$ & Ligh end of RAMP protocol at $5.0 \mathrm{G}$ & $0.55-0.57$ \\
\hline VIB & Filling in particles under continuous vibration & $0.60-0.62$ \\
\hline
\end{tabular}

Figure 2.5: Overview of all protocols for preparation of tetrahedra packings, with the accessible range of packing fractions.

\subsubsection{Initial (loose) preparation}

The aim of this preparation technique is to find the mechanically stable packing with the lowest density, in analogy to the random loose packing density of spheres. A loose packing is prepared by inserting a hollow cardboard cylinder into the container, and filling in the particles through a funnel (Fig. 2.6a). The cardboard cylinder is then slowly lifted, to allow a gentle relaxation of the particles (Fig. 2.6b). This procedure creates loose tetrahedra packings (Fig. 2.6c), with reproducible packing fractions of $\Phi=0.472 \pm 0.003$, which are prone to accidental rearrangements, and thus must be handled with care.

\subsubsection{Compaction by tapping (TAP)}

In the spirit of tapping experiments $[55,66]$, the protocol TAP is defined by a train of separated sine pulses with a duration of $50 \mathrm{~ms}$ and a repetition rate of $3 \mathrm{~Hz}$. The pulse duration is chosen sufficiently long to minimize the distortion between input signal and transduced mechanical excitation (Fig. 2.7). On the other hand, the pulse length is limited by the mechanical limit of the shaker elongation: A longer pulse has a lower frequency $f$, hence a desired intensity $\Gamma$ can only be achieved by increasing the amplitude, according to Eq. 2.1. Higher repetition rates would decrease overall acquistion time, but do not allow the packing to come to rest between the pulses, leading to unwanted effects such as surface waves. 


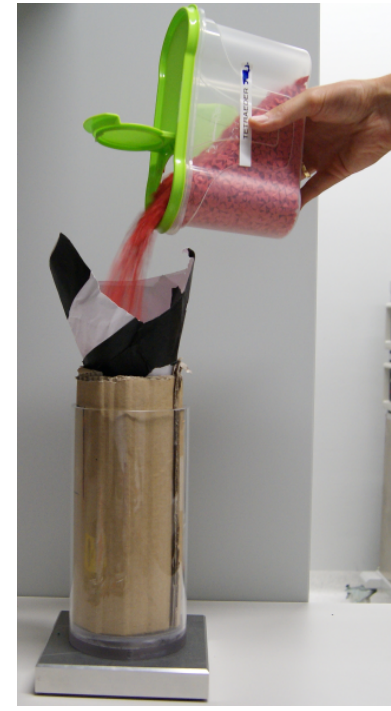

(a)

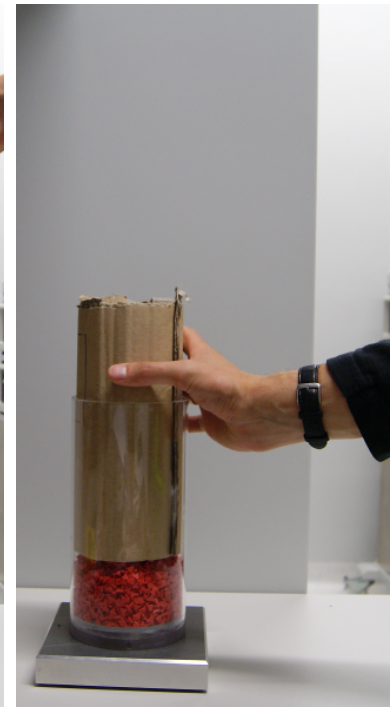

(b)

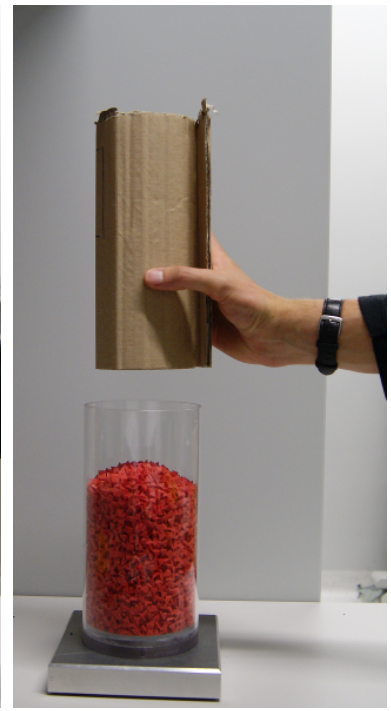

(c)

Figure 2.6: Preparation of loose tetrahedra packings

The input signal and the corresponding accelerometer signal are compared in Fig. 2.7. The movement of the exciter table does not follow the input signal immediately, but heads first towards the opposite direction and responds to the pulse with a phase delay of approx. $\pi / 2$. After the tap, the table recovers its equilibrium position within 20-30 ms. These differences between signal and mechanical excitation are attributed to the inertia and the non-smooth input signal (discontinouity of the first derivative). If the intensity is sufficiently high $(\Gamma \geq 4)$, a strong, noisy signal is detected after the pulse, because the packing takes off from the bottom shortly and crashes down again [66].

\subsubsection{Annealing procedure (RAMP)}

The RAMP protocol follows closely the "Annealing" procedure originally introduced in the seminal paper by Nowak et al.[55]. It is intended to study the possible range of steady state packing fractions $\Phi_{\infty}$. Following Nowak et al.[55], the tap intensity $\Gamma$ is increased stepwise from 0.25 to 5 , decreased back to 0.25 , and increased to 5 again. At each step, $10^{4}$ taps are applied and the resulting $\Phi_{\infty}$ is measured via laser triangulation. Fig. 2.8 sketches the evolution of the control parameter vs. runtime of the experiment for a complete RAMP cycle. 


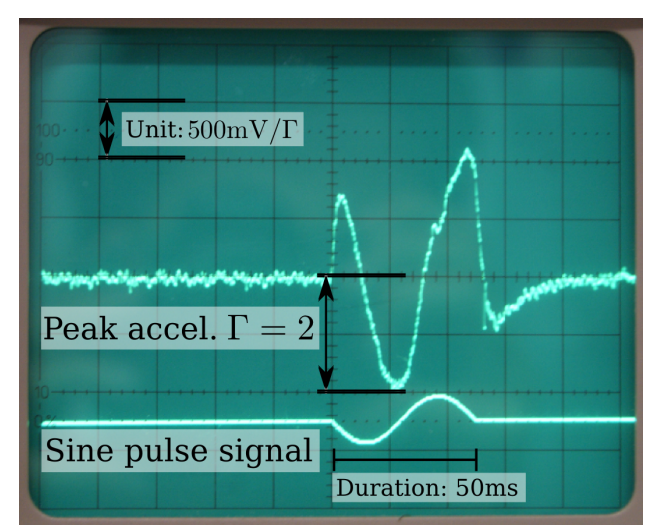

Figure 2.7: (Original sine pulse signal from function generator (duration $50 \mathrm{~ms}$, amplitude $232 \mathrm{mV}_{\mathrm{pp}}$ ) and acceleration ("Peak accel.") measured simultaneously at the shaker table.

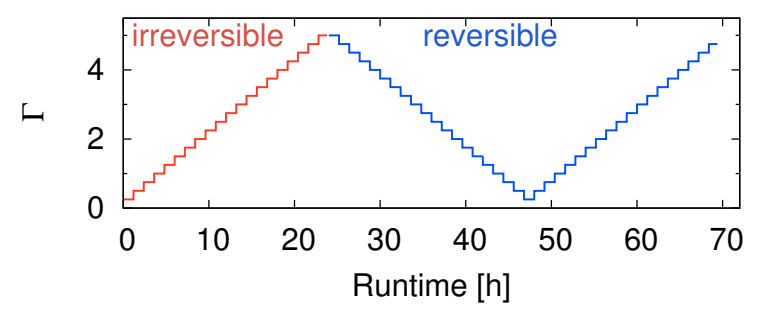

Figure 2.8: Protocol RAMP: Acceleration is first increased step-wise from $\Gamma=0.25$ to $\Gamma=5$, then alternately ramped down and up between these bounds. One full cycle is shown, with a total runtime of 70 hours. On each plateau of the steps, $10^{4}$ taps at the current $\Gamma$, with a repetition rate of $3 \mathrm{~Hz}$ are applied.

\subsubsection{Vibration protocol (VIB)}

The possible configurations of packings prepared by the above protocols are limited, because particles get jammed due to gravity. While the surface particles are still mobile, possible rearrangements in the bulk region are restricted by geometric frustration and pressure of the pile.

Therefore, the protocol VIB is introduced, which is inspired by epitaxial growth. Epitaxy is a commonly used technique for semiconductor production and refers to the growth of a crystalline film from a gas/liquid phase on a substrate with fitting lattice parameters [93]. To this aim, it is necessary to heat the substrate for enhanced surface diffusion and hence growing of crystalline layers. The growth rate is limited by the temperature-dependent reaction kinetics and the diffusion constant of the gas phase. In analogy, growing a crystal from granular matter needs constant mechanical agitation while the grains are deposited by gravity. The deposition rate needs to be sufficiently low, so that dense configurations can arrange layer by layer. Us- 
ing this preparation technique, crystalline packings of spherical beads [60] or dense ellipsoid packings were found [18]. Even the growth of a quasicrystal [26] is possible, if a appropriate seed layer is prepared at the container bottom ${ }^{2}$. In the protocol VIB, tetrahedra particles are poured manually at a rate of approximately 15 particles/sec into the container, while continuous vibration is applied. The VIB parameters are a frequency $f=100 \mathrm{~Hz}$ and a intensity of $\Gamma=5$.

\subsection{X-ray tomography}

After X-rays were discovered by Wilhelm Röntgen in 1895 [70], they were soon applied for imaging internal structures, such as bones or for the "shoefitting fluoroscopes". Apart from medical X-ray usage, the development of $\mathrm{X}$-ray tomography in the last decades has opened another field of applications for the non-destructive 3D analysis in material science or archeology $[83,50]$. Here, an X-ray computed tomography system is employed to record a tomographic $3 \mathrm{D}$ representation of the granular tetrahedra packings.

\subsubsection{Nanotom setup}

The present X-Ray tomography device is a commercially available "Nanotom" manufactured by General Electric (formerly phoenix x-ray). Fig. 2.9 depicts a schematic overview of the setup, consisting of the X-ray tube, the sample table and the detector unit. The X-rays emitted from the tungsten target (anode) have a polychromatic spectrum, that is composed of Bremsstrahlung and the characteristic radiation. While the packing sample is turned on a CNC table in small angle increments $\left(\sim 0.15^{\circ}\right)$, a large number of absorption images (radiograms) is recorded, which are later reconstructed as a volume dataset (tomogram).

\subsubsection{Choice of optimal tomography parameters}

In the following section, four different aspects (I-IV) of the tomographic acquisition are outlined. I draw an analogy to classical photography, where a good picture relies on the optimal choice of:

I) Lighting conditions $\Leftrightarrow$ X-ray photon flux

II) Perspective/zoom on the motif $\Leftrightarrow$ Acquisition geometry/magnification

III) Exposure and aperture $\Leftrightarrow$ Detector timing

IV) Development of negatives $\Leftrightarrow$ Tomographic reconstruction

\footnotetext{
${ }^{2}$ Pablo Damasceno, private communication
} 


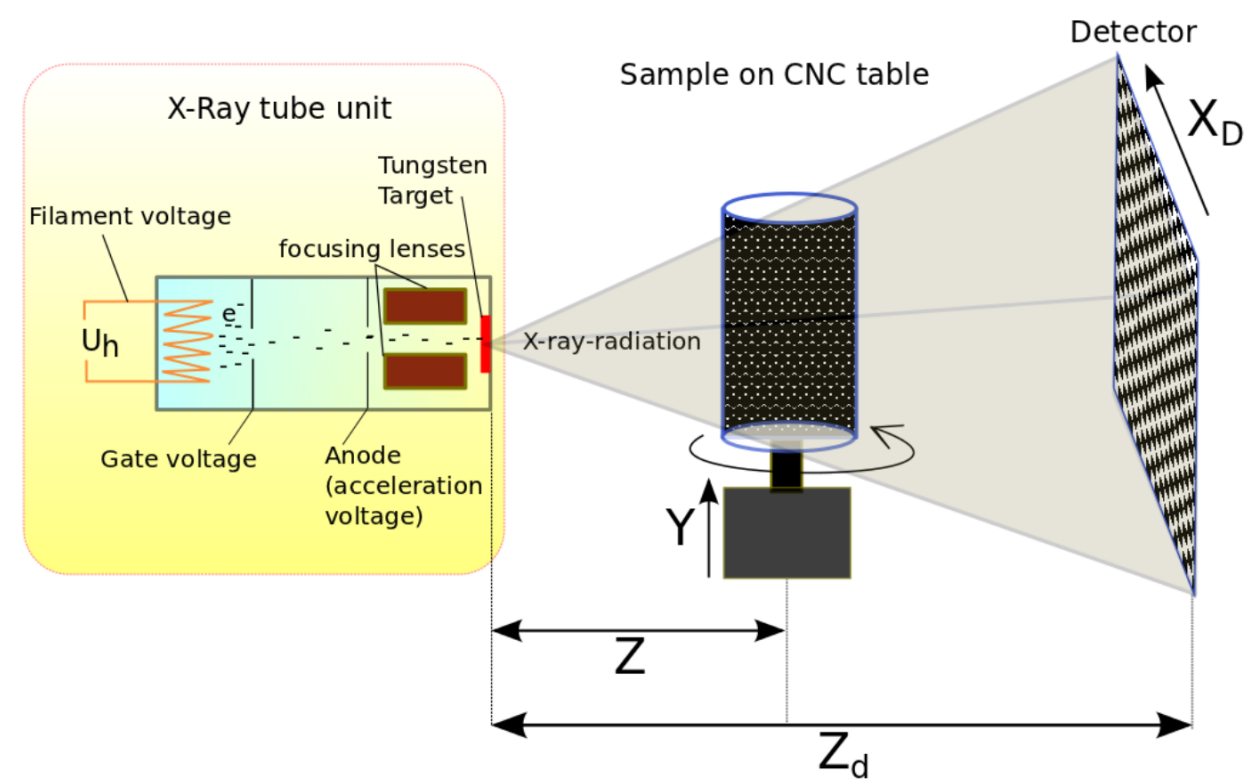

Figure 2.9: Nanotom setup (from left to right): The filament voltage $U_{h}$ controls thermionic emission of electrons from the filament. The gate voltage is biased to a negative potential relative to the catode (filament), and serves as a Wehnelt cylinder for controlling and focusing the electron cloud. X-radiation is created by guiding the electron beam onto a tungsten transmission target (anode), from where radiation spreads ("fan-beam geometry"). The ratio $Z_{d} / Z$ determines the geometric magnification. 
Between initial X-ray generation and tomographic reconstruction of the volume, different parameters need to be optimized for the best radiogram and tomogram quality.

\section{Stage I: X-ray settings and resolution}

The diameter of the focal spot on the target, where radiation is emitted, is the lower limit of the attainable voxel resolution. Four different "modes" $(0,1,2,3)$ are available in the control software, ranging from 0 with a focal spot of $\sim 3 \mu \mathrm{m}$, to the "nanofocus" mode 3 with a spot size of $\sim 1 \mu \mathrm{m}$. However, the highest focus mode comes at the cost of total X-ray brightness, because it limits the maximum power to avoid burn-ins at the target. Therefore, for a typical granulate sample with a relatively large diameter of $1-10 \mathrm{~cm}$ and grain sizes $>100 \mu \mathrm{m}$, mode 0 is preferred.

The optimal combination of acceleration voltage $U$ and filament current $I$ depends on the sample material and its X-ray transmission characteristics. In general, samples with high absorption coefficient (like glass, plastic, or iodine contrast agent) need higher voltages $(120-170 \mathrm{kV})$ than biological samples $(<80 \mathrm{kV})$ to achieve the same tomogram quality. A commonly used metric for evaluation of image quality is the contrast $K$, measured on the distribution of grey values $g$ :

$$
K=\frac{\max (g)-\min (g)}{\max (g)+\min (g)}
$$

From a practical point of view, a wide range of grey values is desirable, which implies a high brightness and a large difference between material and background absorption. The following scan procedure of the optimal $U, I$ settings for tetrahedra packing samples (material: polypropylene) was devised: Firstly, calibration images ("Gain") in the voltage range from $80 \mathrm{kV}$ to $170 \mathrm{kV}$ are taken without the sample. At each voltage step, the current $I$ is adjusted so that the measured average grayvalue $\langle g\rangle$ is constant and roughly in the middle of the dynamic range of $0 \ldots 4095$ (here, $\langle g\rangle=2300$ was chosen). Fig. 2.10a shows the relationship between $U$ and $I$; the kink at $120 \mathrm{kV}$ can be attributed to the emergence of characteristic radiation in the spectrum. Secondly, the sample is mounted and radiograms are recorded in the same range of voltages $U$ as calibrated by the gain images. The current $I$ is adjusted to yield constant power $P=U I$ at the target (here, $P=22.4 \mathrm{~W}$ was set).

In principle, the range of recommended power settings has two limitations, which concern filament wear-off and occurence of X-ray breakdowns: Increasing the current creates more X-ray photons and improves overall image quality (unless oversaturation of the detector is reached), but on the other hand, the lifetime of the filament decreases faster than linear. At 
high voltages $>175 \mathrm{kV}$ (close to the maximum of $180 \mathrm{kV}$ ), the probability of breakdowns (short-circuit in the vacuum tube, or in the high-voltage generator) increases. Breakdowns cause the system to immediately shut down X-radiation and ramp it up slowly again, which may lead to substantial brightness variations between radiograms, deteriorating the final reconstruction.

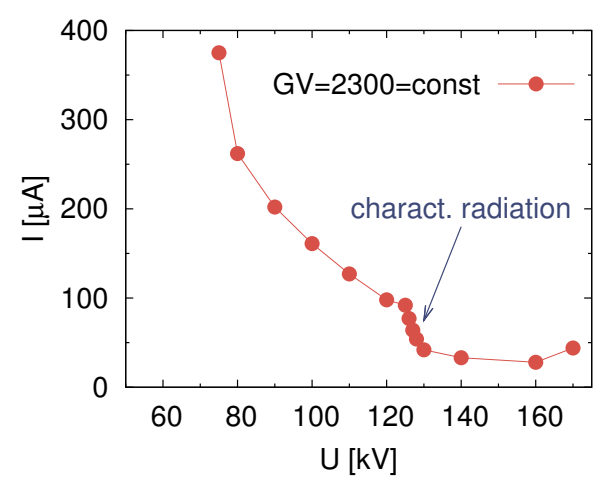

(a)

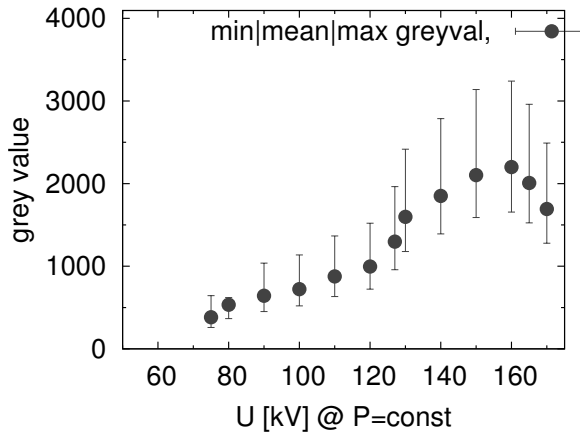

(b)

Figure 2.10: (a) Dependency of filament $I$ current vs. acceleration voltage $U$ for constant average brightness $\langle g\rangle=2300$. (Gain calibration images without sample). (b) Determination of optimal acceleration voltage: Radiograms of the samples are recorded for a range of acceleration voltages $U$, under the condition that the power $P=U I$ is fixed at (that is $I=P_{\text {const }} / U$ ). The optimal brightness and contrast is found for $U=160 \mathrm{kV}$. The increase between 120 and $130 \mathrm{kV}$ is caused by the emergence of the characteristic tungsten peaks.

\section{Stage II: Sample and detector position}

The geometric magnification $G$ in the fan-beam setup is determined by the ratio between detector distance $Z_{d}$ and sample distance $Z$ to the X-ray source as shown in Fig. 2.9: $G=Z_{d} / Z$. That implies that the same magnification (e.g. $G=2$ ) can be achieved by different absolute positions, like the ratios $400 \mathrm{~mm} / 200 \mathrm{~mm}$ or $500 \mathrm{~mm} / 250 \mathrm{~mm}$. In this case, the closer (smaller value) of the detector position should be chosen, because of the larger photon flux.

\section{Stage III: Detector settings (image acquisition)}

At the detector, X-ray photons are converted by a scintillator layer into visible light, which is then recorded by a CCD chip. The detector has the dimensions of $115 \times 115 \mathrm{~mm}^{2}$, and a resolution of $2304 \times 2304$ pixels with a pixel size of $50 \mu \mathrm{m}$. In principle, the photon flux $P$ (number of photons per time unit) determines the overall image quality, because the noise level decreases with $1 / \sqrt{P}$. The detector settings offers two possibilites to increase 
the number of incoming photons: Firstly, by binning neighboring pixels into $2 \times 2$ or $4 \times 4$ super-pixels, which increases brightness 4 -fold resp. 16 -fold, but reduces resolution ( $\mu \mathrm{m} /$ voxel) by a factor of 2 resp. 4 . Secondly, the exposure time for a single radiogram can be varied between $1 / 8 \mathrm{~s}$ and $4 \mathrm{~s}$. Further reduction of the noise level is achieved by averaging over a number of single exposures ("Averages") for each radiogram. The "virtual sensor" allows to move the detector in horizontal and vertical direction during tomography, so that the detector size can be extended by $3 \times 3$ tiles (Virtualsensor and MultiScan options). Lastly, the total number of radiograms determines the angle increment of the rotation: As a guideline, the number of radiograms should be at least the image width of the radiogram in voxels.

All in all, the specific choice of settings depends on these four goals: high resolution (=small field of view), low noise, short acquisition time and low material wear-off. For the tetrahedra packings with a cylinder diameter of $11 \mathrm{~cm}$, a large field of view, respectively a low geometric magnification of $G=2$ is needed. A detector binning of $2 \times 2$ is chosen, which yields a resolution of $50 \mu \mathrm{m}$ per voxel at this magnification. The detector tiling is set to $2 \times 2$, so that the imaged part of the sample amounts to approx. $11 \mathrm{~cm} \times$ $11 \mathrm{~cm} \times 10.3 \mathrm{~cm}$. In the following reconstruction step, a downsampling to $50 \mu \mathrm{m}$ is performed, so that the final size of the volume is $1107 \times 1107 \times 1026$ voxels. The lower height dimension is due to a necessary overlap between tiles for stitching the images taken at the different virtual positions (tiles). The optimal X-ray settings are determined as $U=160 \mathrm{kV}$ and $I$ in the range of $140-160 \mu \mathrm{A}$.

\section{Stage IV: Tomographic reconstruction}

The reconstruction of a tomographic volume is a typical inverse problem: We want to obtain the density distribution $f(x), \quad f \in \mathbb{R}, x \in \mathbb{R}^{3}$ of an 3D object, but only the radiographic absorption images (shadow projections) from different angles are known. The mathematical foundations are based on the Radon transformation [65], which is a set of line integrals of a function along different angles. For illustration, consider the two-dimensional problem first: The X-ray path is a line passing through the sample with varying density $f(x, y)$, and is parametrized by the distance $D$ to the origin and the angle $\theta$, as sketched in Fig. 2.11. The actual X-ray absorption depends exponentially on the path length (Beer-Lambert law), but this effect is neglected here. The Radon transform $g(D, \theta)$ is formulated as follows:

$$
g(D, \theta)=\int_{\mathbb{R}}^{2} f(x, y) \delta(\theta-(x \cos \theta+y \sin \theta)) \mathrm{d} x \mathrm{~d} y
$$

A commonly used technique for tomogram reconstruction is the filtered backprojection [10]. A "naive" or direct backprojection would just overlay the recorded Radon transforms from Fig. $2.12 \mathrm{~b}$ and recreate the image. 


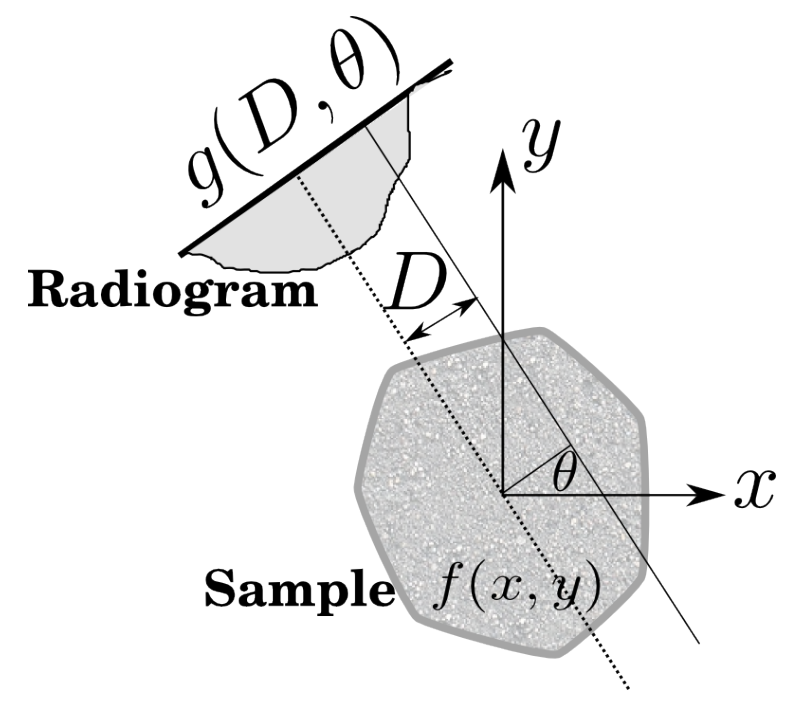

Figure 2.11: The radon transformation $g(D, \theta)$ corresponds to a radiogram recorded under the angle $\theta$ and at the beam offset resp. detector position $D$. The sample has a arbitrary density distribution $f(x, y)$.

However, the outcome is a strongly blurred version (Fig. 2.12c) of the original image (Fig. 2.12a). It can be shown that the direct backprojection convolutes convolves any point in the image $f(x, y)$ with a point-spread function of the form $1 /|(x, y)|$. The filtered back-projection accounts for this problem by filtering in frequency space. A common filter is the Ram-Lak filter, which has a frequency response of $|f|$, that is, low image frequencies are suppressed. In such a way, the original image can be reconstructed (Fig. 2.12d). The ramp filter induces artefacts (here: caused by the sharp edges), which can be reduced with more sophisticated filtering techniques (windowing).

Further details on reconstruction algorithms in 2D and 3D can be found in the Refs. [10, 50]. For the practical reconstruction of radiograms, a proprietary algorithm based on a cone beam filtered backprojection [22] is provided by with the Nanotom software. 


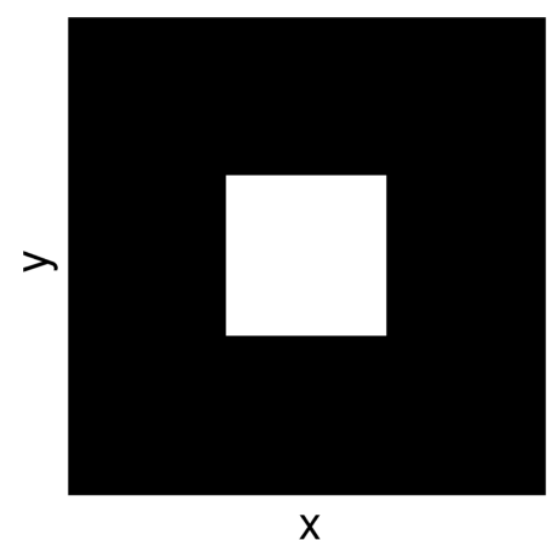

(a)

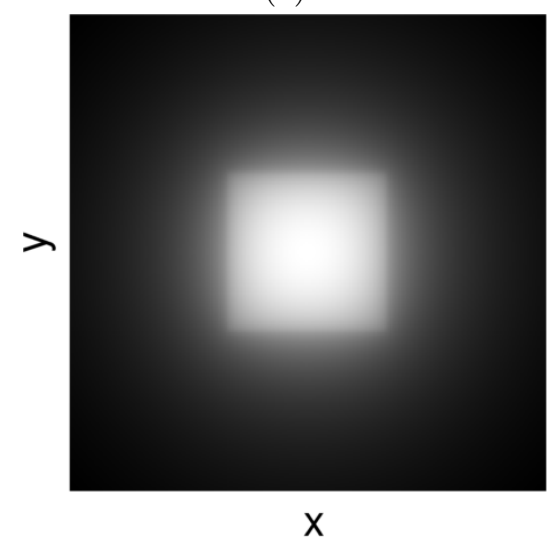

(c)

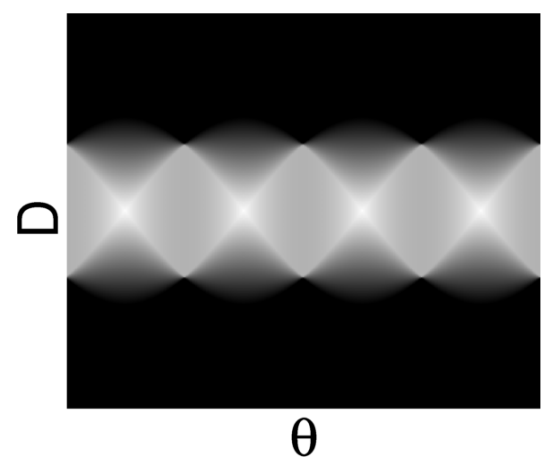

(b)

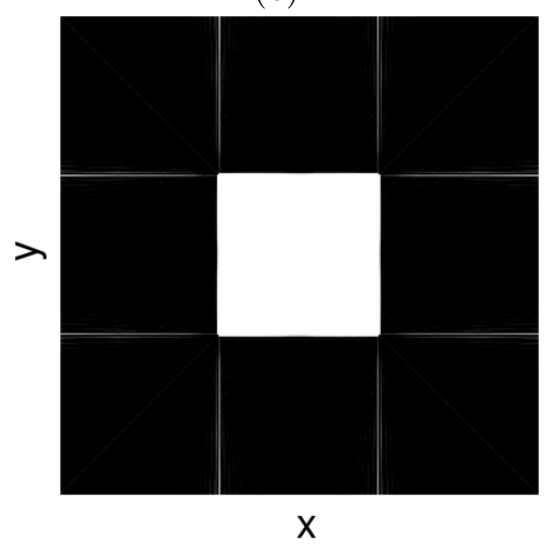

(d)

Figure 2.12: (a) Binary image as sample distribution $f(x, y)$ (b) Radon transform $g(D, \theta)(\mathrm{c})$ "Naive" backprojection of $g(\mathrm{~d})$ Filtered backprojection using a ramp filter. Strong contrast enhancement to make the sharp edge artefacts visible 


\section{Chapter 3}

\section{Processing of tomographic volumes}

The tomographic acquisition is the beginning of an image processing chain with increasing complexity and information level, while the amount of data is reduced by 3 orders of magnitude. Fig. 3.1 illustrates this process, starting from a simple photograph (Fig. 3.1a). The first part of this chapter describes the workflow from the tomographic density data (Fig. 3.1b) to the geometric representation of tetrahedra (Fig. 3.1c). The latter parts focus on the analysis of these geometric tetrahedra with respect to contact numbers, constraints and Voronoi volumes.

The reconstructed volume has the dimensions of $1107 \times 1107 \times 1026$ voxels, a spatial resolution of $100 \mu \mathrm{m} /$ voxel, and a gray value range of 16bit, resulting in a file size of $\sim 2.5$ GByte per sample. At the end of the processing chain, the structured variable TCSet contains all particle centroids, vertices and their associated edges and face normals ( $\sim 3 \mathrm{MB}$ per sample). This data structure is suitable for analysing local contacts and contact geometries (see Appendix A.2).

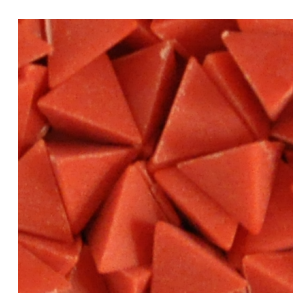

(a)

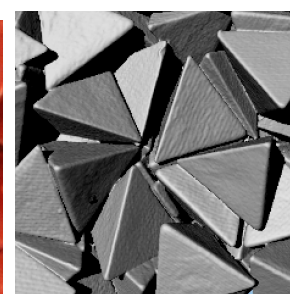

(b)

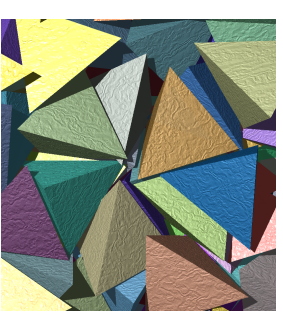

(c)

Figure 3.1: (a) Photograph of a packing surface, no 3D information. (b) Tomographic volume, 3D material density information (c) After particle registration, the geometric representation of the particles is obtained (colored by orientation) 


\subsection{Particle detection}

Since polyhedra packings pose specific challenges to the particle detection, a complete image processing workflow was designed and implemented in MATLAB. The following notation uses the terms "image" and "volume" interchangably for the 3D-dataset, whereas 2-dimensional cuts, fixing one coordinate (e.g. the $z$-coordinate) are denoted as "slices" (e.g. "slice at $z=200 ")$. Subsets of a image are typically referred to as "Region Of Interest" (ROI) within the image. All coordinates are given in voxel units, with a isotropic voxel spacing of $100 \mu \mathrm{m}$. The physical particles (tetrahedra and octahedra) have a sidelength of $a=7 \mathrm{~mm}$ or 70 voxels. In binary images, by convention, white (foreground) corresponds to the particle phase, and black (background) to the air phase. The reader is referred to Appendix A.1 for an introduction to morphological image operations.

\subsubsection{Algorithm overview}

Figure 3.2 outlines the image processing workflow from the reconstructed tomographic volume to the particle representation by geometric tetrahedra. It consists of four main stages, with increasing complexity and information level:

I) Grey-value based segmentation: Reduction to 8-bit, binarization $\rightarrow$ binary volume

II) Region-based segmentation: Watershed labeling

$\rightarrow$ segmented regions

III) Registration: Numerical maximization $\rightarrow$ registered particles

IV) Contact detection: Computational geometry

$\rightarrow$ packing structure

\subsubsection{Preprocessing and filtering}

The Nanotom acquisition software datosx-acq 1.6 offers a "Multiscan" option, which creates two tomography datasets, representing a lower and a upper part of the cylindrical sample. The optimal vertical stitching position of these subvolumes, is determined by the vertical shift between the two tomographies, and is verified by maximizing the cross-correlation between associated image slices of the two subvolumes (accuracy: 1 voxel).

The first processing step reduces the dynamic range from 16bit to 8bit in order to save storage and processing time. However, a direct mapping (division by 256) does not approximate the original dynamic range [0 ..65535] 


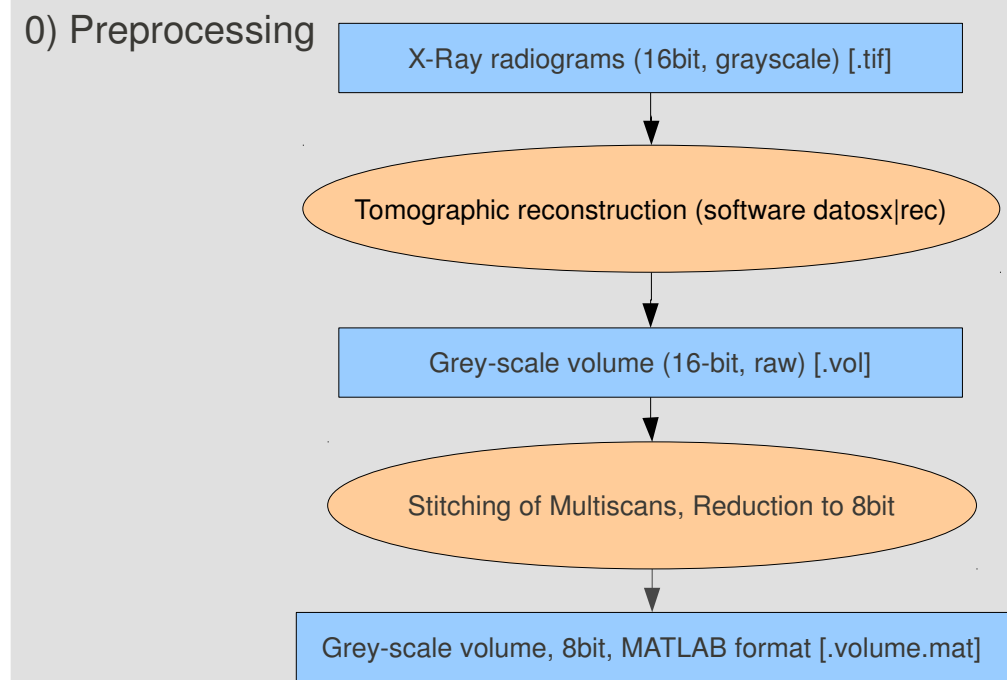

\section{I) Gray-value segmentation}

\section{$\nabla$}
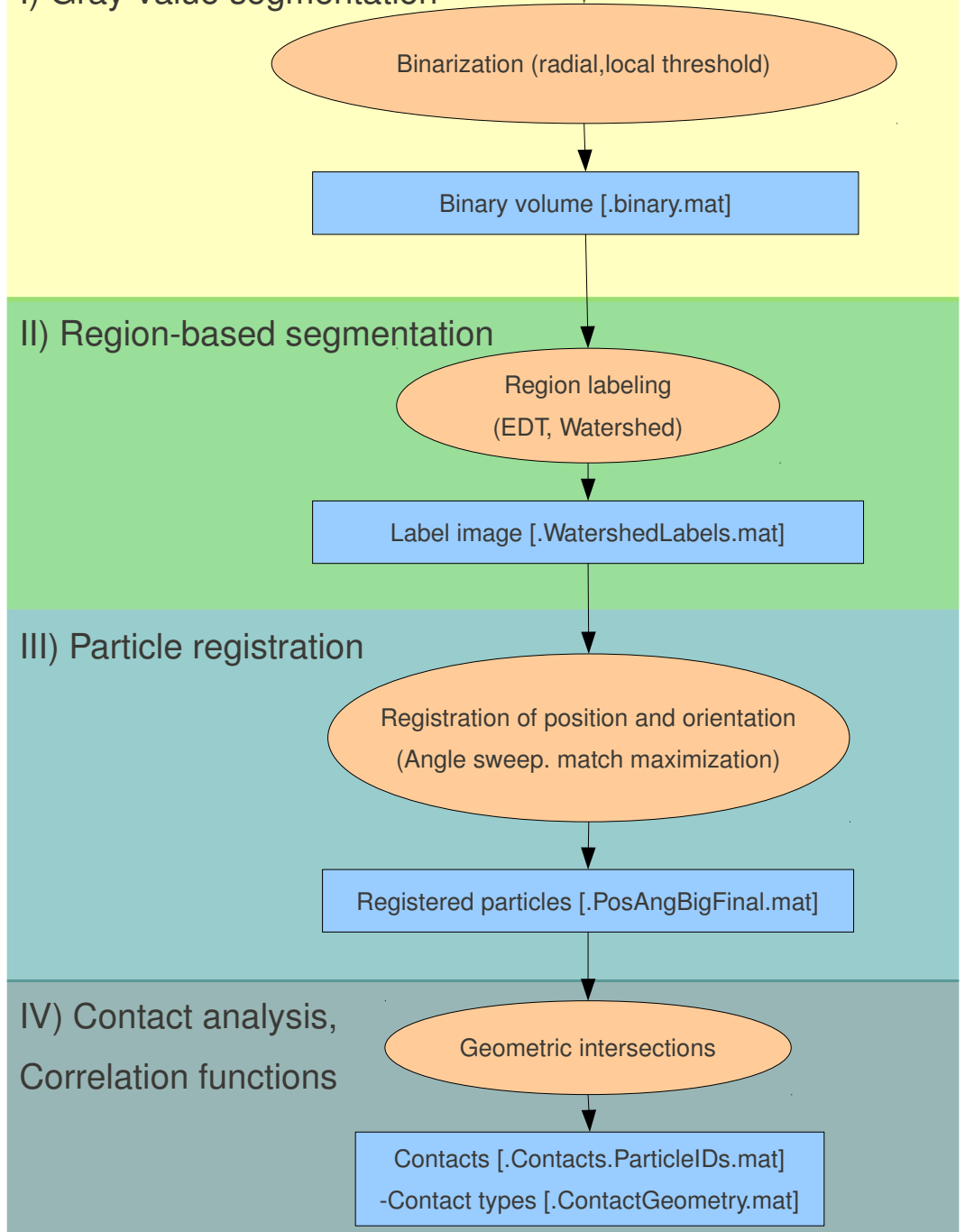

Figure 3.2: Schematic image processing workflow. Intermediate output files are shown as blue boxes, data processing steps as orange ellipses. 

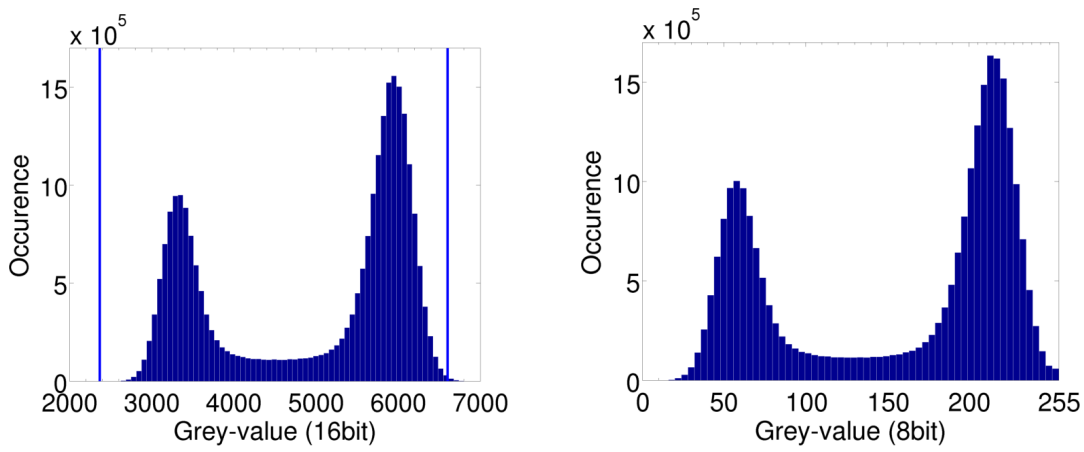

Figure 3.3: (a) Original 16-bit histogram, lines indicate cutoff values at 0.1 and $99.9 \%$ of the cumulative histogram. (b) Transformed 8-bit histogram

well, because the grey-values are not evenly distributed in the interval. Instead, the cumulative original histogram is analysed and a cutoff interval $[0.1 \%, 99.9 \%]$ is chosen, corresponding to low resp. high grey value cutoffs of $\sim 2000$ resp. 6000 (effectively 12-bit). Mapping this range linearly to the 8-bit interval $[0 \ldots 255]$ preserves the dynamics well, as Fig. 3.3 shows.

\subsubsection{Grey-scale based segmentation}

\section{Introduction and related work}

The process of partitioning an image in disjoint sub-regions is generally referred to as segmentation. Typically the first segmentation task involves binarization, that is the distinction between foreground and background pixels according to the brightness (grey-value) of a voxel $\mathbf{x}$. The human eye performs this segmentation easily, but automated and robust image segmentation is generally not a trivial task. Binarization reduces the dynamic range from a range of grey-values to 1-bit, but many processing steps like the identification of connected components or distance transformations rely on the binary image (see Sec. 3.1.4.)

\section{Otsu's global threshold}

The segmentation threshold can be computed globally or locally. A still widely used global binarization method was proposed by Otsu [57]: It assumes a bimodal grey-value distribution and finds the optimal threshold $\Theta$ by maximizing the variance $\sigma_{B}$ between both classes. If $l$ discrete grayvalue levels are present, we denote the distribution by $p(l)$ with its mean $\mu$, and the subsets of foreground $p_{f}(l)=p(l \mid l>=\Theta)$ and background voxels $p_{b}(l)=p(l \mid l<\Theta)$ with the corresponding mean values $\mu_{f}$ and $\mu_{b}$. The distributions are normalized by their total number of voxels so that $\sum_{i} p_{i}=1$. The optimal threshold $\Theta$ is then found by maximizing the following expression for the intra-class variance: 

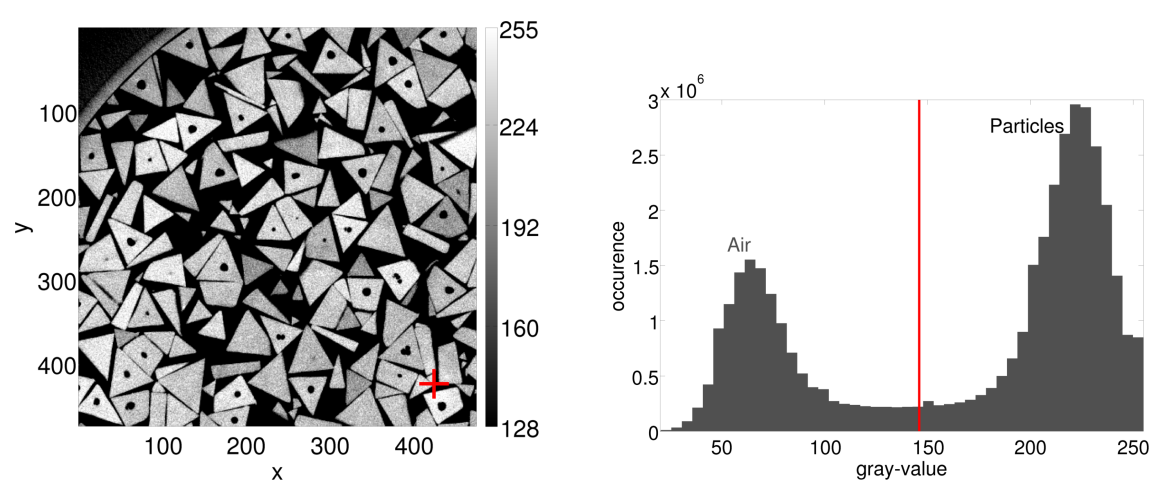

Figure 3.4: Left: Selected ROI of tetrahedra packing, 8-bit grey-scale (Sample TAP100000), units in voxels (slice at $\mathrm{z}=200$ ). Red cross marks the radial center, upper left corner shows the cylinder wall. Right: Histogram of grey-values in central region of volume, red line: Otsu's threshold

$$
\sigma_{B}=\left(\sum_{l<\Theta} p_{b}(l)\left(\mu_{b}-\mu\right)^{2}+\sum_{l>\Theta} p_{f}(l)\left(\mu_{b}-\mu\right)^{2}\right)
$$

In the case of a 8-bit image with 256 grey-values, the threshold is simply determined by evaluating $\sigma_{B}$ for all $\Theta \in 0 \ldots 256$ and choosing the maximum. A iterative algorithm for maximizing $\sigma_{B}$ by the above expression was tested as well (Mardia-Hainsworth), but results were equivalent to those of Otsu's method, in accordance with other experimental results [89]. Fig. 3.4 shows a selected grey-value slice of a tetrahedra packing, and the corresponding histogram of the volume. The two peaks are identified as foreground and background and are clearly separated by Otsu's threshold.

\section{Radially varying threshold}

Depending on the beam energy, the tomograms may exhibit a pronounced radial brightness variation due to beam hardening artefacts: The path length dependent X-ray absorption is highest for rays passing through the sample center, and lowest for the cylinder wall, so that the apparent absorption is higher in the center. Since the grey-value representation maps apparent density to brightness, the brightness decreases radially. This can be accounted for by a radially varying threshold, where each local threshold is chosen by Otsu's criterion. The area of each ring is kept constant, leading to radially decreasing step sizes, as Fig. 3.5 illustrates.

\section{Local threshold}

A close look at the volume slices reveals that substantial local brightness variations are present in the tomographic volume, which are probably at- 

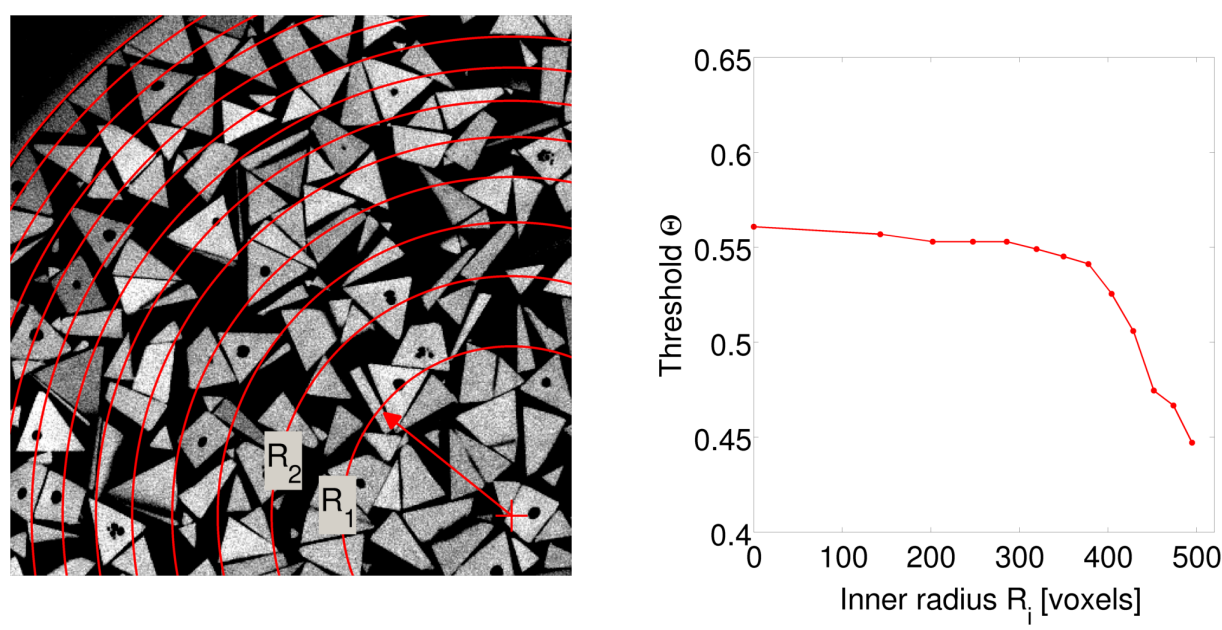

Figure 3.5: Radial rings for local thresholding

tributable to material density variations and

These figures indicate that both the global and the radially dependent threshold do not perform well in segmenting foreground from background. A better approach is the computation of local thresholds $\Theta(\mathbf{x})$, in a window $W$ of appropriately chosen size. This technique was popularized as Niblack's method [53] and an efficient implementation for arbitrary window sizes is available [75]. While Niblack's method determines the local threshold from mean and variance within the window, a modified version has better performance and is comparably accurate [49]. The latter version only estimates the mean grey-value $\mu_{W}$ within the sliding window $W$ and sets the voxel value to 0 if it is at least $t$ percent below the mean, and to 1 otherwise. In addition to the window size $W$, the parameter $t$ influences how much of the fuzzy object boundary is assigned to the object or background. A drawback of the local binarization is the manual choice of window size and threshold.

Optimal filter parameters were determined by inspecting the final segmentation quality and minimizing over- and undersegmentation across tetrahedra, octahedra and mixed samples. It is recommended to chose the window size according to the typical size of particles in the volume, which is approximately 57 voxels for tetrahedra (particle diameter estimate: distance from face to opposite corner). We found however more robust results with a smaller window of size $W=[25,25]$, and a threshold of $t=10 \%$. Smaller window sizes or lower threshold values tend towards oversegmentation, whereas a too large window does not account for the particle brightness variations in the mixed samples, resulting in undersegmentation (merging) of particles.

The local thresholding is known to assign voxels in extended background regions wrongly to the foreground, because they fulfil the threshold criterion 


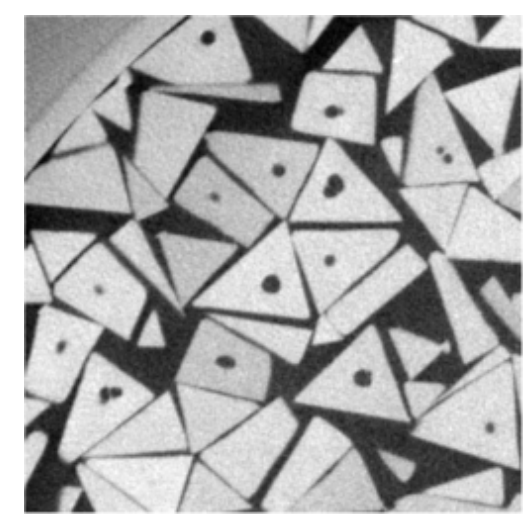

(a) Grey-scale (8-bit)

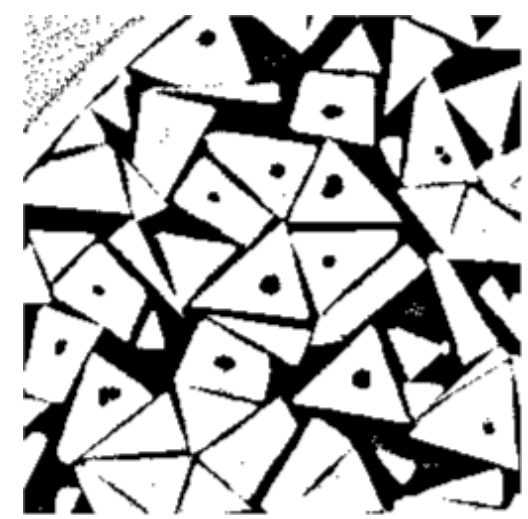

(c) Locally binarized $I_{B}^{\text {loc }}$

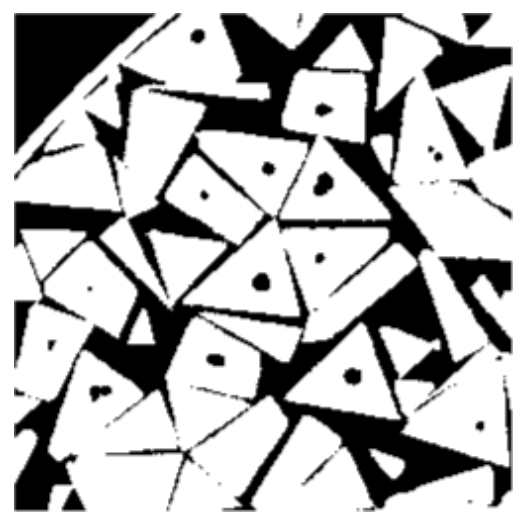

(b) Radially binarized $I_{B}^{r a d}$

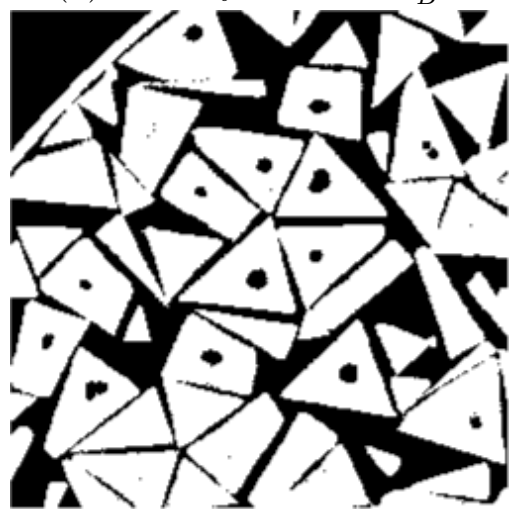

(d) Masked binary image $I_{B}^{\text {loc }} \cap$ $I_{B}^{r a d}$

Figure 3.6: Two-stage binarization: (a) Original grey-scale image slice, left upper corner shows outside part of cylinder. (b) Radial binarization $\left(I_{B}^{r a d}\right)$ with Otsu's threshold in each ring leads to merging of particles (threshold too low). (c) Locally binarized image $I_{B}^{\text {loc }}$ preserves foreground details, but actual background pixels are assigned to foreground (d) Masked binary image $I_{B}^{l o c} \cap I_{B}^{\text {rad }}$ yields good binarization quality, compared to the grey-scale image 
locally. A straightforward solution is to mask the locally binarized imaged $I_{B}^{l o c}$ with the radially binarized version $I_{B}^{R a d}$, that is: $I_{B}^{l o c} \cap I_{B}^{R a d}$, thus removing the spurious pixels in the background regions while preserving the details of $I_{B}^{\text {loc }}$ in the foreground, as Fig.3.6 illustrates.

\subsubsection{Region-based segmentation}

Contrary to segmentation by grey-value, region-based techniques construct segments of interest according to connectedness or neighborhood relations ${ }^{1}$. The present implementation process-flow is outlined in Fig. 3.7 and referred to in the subsequent sections.

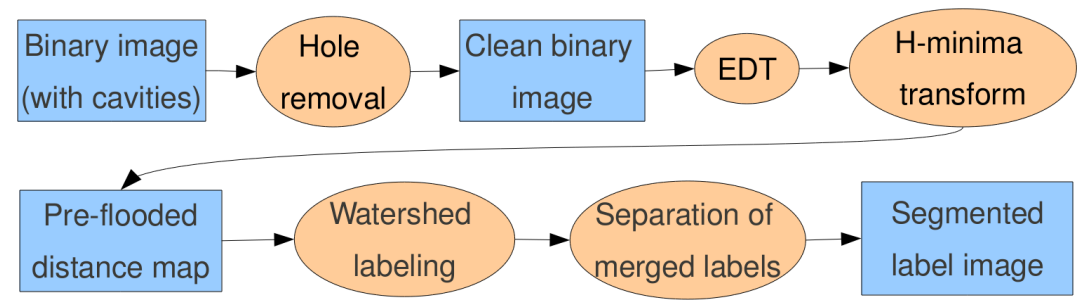

Figure 3.7: Schematic process flow of region segmentation.

\section{Hole removal}

The polypropylene particles contain shrinkage cavities, which are inherent to injection molding, and vary widely in size and geometry. Tetrahedra particles have average hole volumes of $(650 \pm 80)$ voxel $^{3}$, while the cavities inside octahedra are typically in the size range of $[100 \ldots 400]$ voxel ${ }^{3}$. Further particle detection steps assume solidity of foreground objects, necessitating the removal of these holes.

This is achieved by labeling the foreground regions of the inverted binary image $\overline{I_{B}}$ (Fig. 3.8a) via the union-find algorithm, and then excluding the largest region, corresponding to the percolated background (see Fig.3.8b). The union of $I_{B}$ and $H$ then gives the hole-filled image $I_{\text {clean }}$.

However, the assumption that the largest region percolates throughout the volume, may not be fulfilled everywhere. In particular, thin separating lines or single voxels, occuring at face-to-face configurations may be filled as well, causing the merging of neighbors in $I_{C}$.

\section{Euclidean Distance transform (EDT)}

The Euclidean distance transform (EDT) is the method of choice for the separation of connected objects $[16,42]$. It is particularly suitable for image segmentation in connection with a subsequent watershed transform (Sec.

\footnotetext{
${ }^{1}$ For a comprehensive survey on different techniques and their performance, see [95]
} 


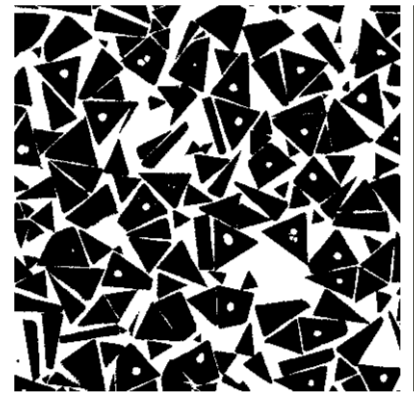

(a)

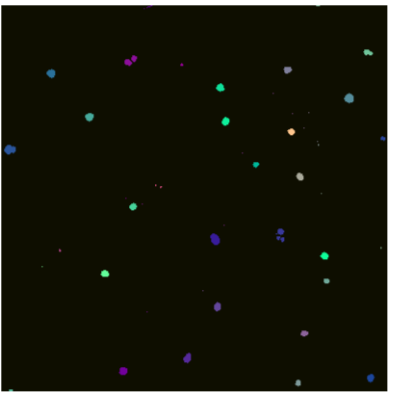

(b)

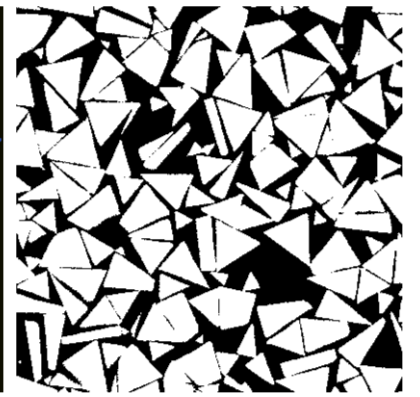

(c)

Figure 3.8: Hole filling procedure. (a) Inverted binary image $\overline{I_{B}}$ (selected ROI), (b) Hole image $H$ : all regions with volume $<80000$ voxels, (c) Resulting "clean" image $I_{C}=I_{B} \cup H$.

3.1.4). In short, a distance map $D$ is created by assigning to each voxel of the foreground voxels the distance of the nearest background voxel. For the present datasets, the EDT is performed on the inverted clean image $\overline{I_{C}}$, and then for each voxel the distance to the background is computed, as Fig. 3.9a shows. The algorithm used in the MATLAB implementation bwdist is based on a optimized nearest neighbor search [23].

\section{Watershed transform}

The watershed transformation (WST) is a indispensable and versatile tool for image segmentation, for a current review see [69].

The working principle is best illustrated by the "flooding" analogy: Consider the gray-scale level of a voxel as a height, then the image can be interpreted as a topological relief. Now holes are pierced into each local minimum, and the relief is immersed into water. Starting from the minima, water progressively floods the so-called catchment basins, and whenever waters from two basins meet, a dam is constructed. These dams are called watershed ridges, dividing the input image into $n_{R}$ disjunct regions, each being assigned a unique label. Internally, the MATLAB implementation of the Fernand-Meyer labeling algorithm [48] is employed for the watershed transform. Basically, the algorithm starts from the local minima and iteratively adds the neighboring pixels with the smallest geodesic distance, which depends on their grey-value (resp. their height in the relief picture).

In the following, the $n_{R}$ watershed regions are just referred to as "labels". The obtained label matrix $L$ is of same size as $I_{C}$, but with the voxel values $\mathbf{x} \in\left(1 \ldots n_{R}\right)$. (Important implementation issue: the class of $L$ must cover the expected number of labels resp. particles, here the unsigned 16bit integer (uint16) is sufficient). 


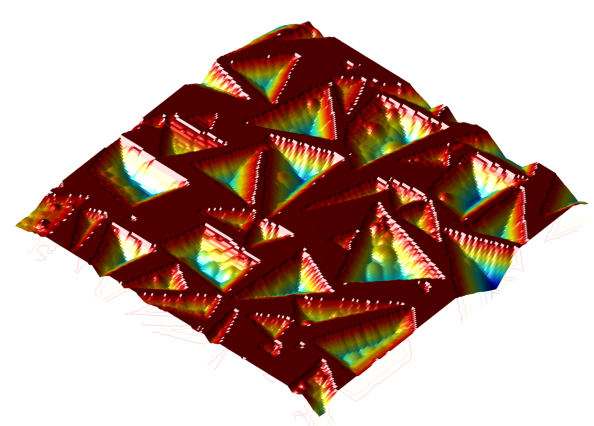

(a)

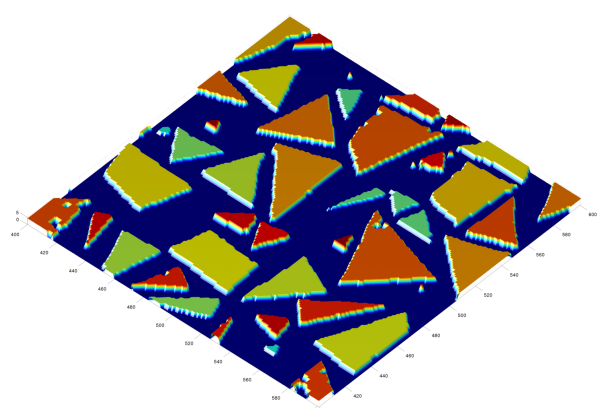

(b)

Figure 3.9: Sample of distance transformation (a) Small ROI of EDT shown as height map, (b) Assigned label matrix $L$ from watershed algorithm.

\section{h-minima transform}

Application of the WST is hampered by its tendency of oversegmentation, that is the errornous splitting of a region containing actually one particle into smaller subregions. This problem is inherent to the algorithm, because each local minimum may be potentially assigned to a region, and each local maximum to a watershed ridge. Therefore, pre-processing is necessary to remove local minima while retaining the particle structure. Smoothing the gradient image by anistropic diffusion yields good results, but comes at the expense of losing image details and a high computational cost $([91,94])$. A better solution is to process the EDT by the h-minima transform, which merges shallow regions with their neighbor regions before the subsequent WST (pre-flooding) [56]. A threshold of $h_{\min }=2.0$ was chosen here, meaning that all basins which are locally separated by weak watersheds of 2.0 or less in altitude, are merged into one basin. A higher setting of $h_{\min }$ increases undersegmentation (merging) of regions, because more watersheds are flooded. However, the exact parameter choice depends on the distribution of wrongly assigned background/foreground voxels of the binarized image, which feed through the EDT and create more local minima in the WST. Therefore, instead of fine-tuning $h_{m i n}$, the binary image should be controlled first for solidity of foreground objects and a low background noise level, and the binarization parameters be changed, if necessary. Fig. 3.10 underlines the importance of the h-minima transform in the segmentation of a tomography of polydisperse gravel (the same gravel as depicted in Fig. 1.1a).

\section{Separation of merged labels}

Large labels, identified by a size threshold, are likely to contain two or more particles. Fig. 3.11 shows a typical distribution of label sizes (=number of label voxels), normalized to the average label size of $\left\langle V_{R}\right\rangle=3.2 \cdot 10^{4}$ 


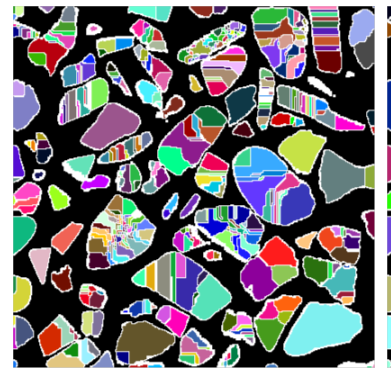

(a)

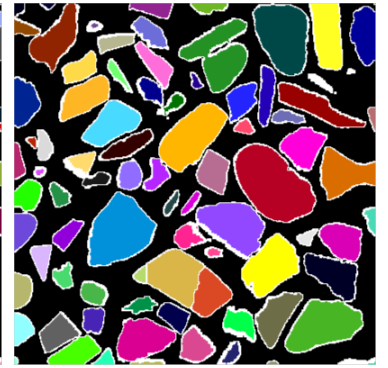

(b)

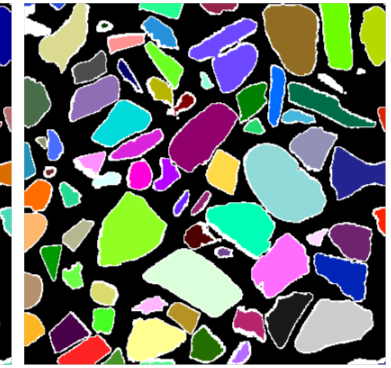

(c)

Figure 3.10: Outcome of Watershed labels for different parameters of the h-minima transformation on a tomography of gravel (a) $h_{\text {min }}=0$ (no transformation): massive oversegmentation (b) $h_{\text {min }}=2$ : reduced oversegmentation, green region at top possibly merged (undersegmented) (c) $h_{\min }=4$ : optimal segmentation

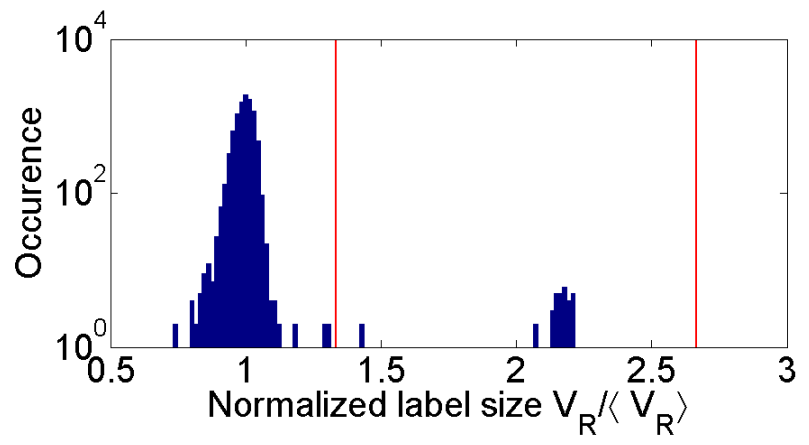

Figure 3.11: Distribution of label sizes $V_{R}$ in a segmented packing (totalling 9293 labels). The region sizes containing doublettes is enclosed by the red lines at $V=\frac{4}{3} V_{R}$ and $V=\frac{8}{3} V_{R}$

voxels. The first peak represents labels containing one particle, the second peak corresponds to doublettes (and so on). All regions (labels) with size $V_{R} \in\left[\frac{4}{3}\left\langle V_{R}\right\rangle \ldots \frac{8}{3}\left\langle V_{R}\right\rangle\right]$ are classified as doublet regions, as illustrated in Fig. 3.11.

In the densest samples, the first Watershed segmentation leaves up to $0.5 \%$ of all labels as dublettes (total number: $\sim 60$ ). The following procedure separates roughly $80 \%$ of these regions, which is a prerequisite for the high detection rate $(>99.80 \%)$ of the final registration algorithm.

In order to split the merged labels, the grey-scale volume is reprocessed by locally enhancing the edges. Edges (respectively planes in 3D) are characterized by a large gradient perpendicular to the edge, and numerous techniques for their detection exist, which vary mainly in the shape of the filter kernel. We employ a 3D version of the Sobel filter here to create a edge image $E$ from the original grey-value volume. $E$ is iteratively binarized with decreasing threshold, producing a sequence of binary edge images $E_{B}$ 


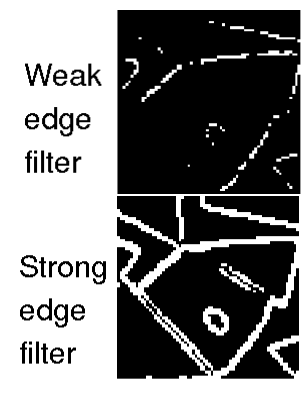

Edge image

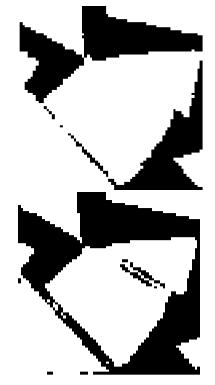

Edges removed from binary

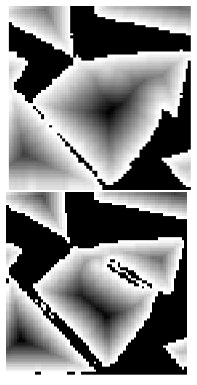

EDT

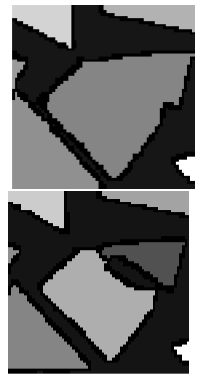

Label image

Figure 3.12: From left to right: binarized edge image $E_{B}$; subtracted $E_{B}$ from binary image; Euclidean Distance transform; Watershed labels image. First row: Original (weak) edge enhancement leads to merging particles, so that correct labeling fails. Second row: Strong edge enhancement allows separation via local maxima in EDT and Watershed labeling.

with increasing edge strength. In each iteration, the edge image is subtracted from the binary image as $I_{C} \cap \overline{E_{B}}$, and fed through the EDT and Watershed transform, until separation in at least two regions is achieved. Fig. 3.13 shows the successful separation of a merged pair of tetrahedra in a face-to-face contact configuration.

\subsubsection{Object registration}

The task of object registration (or recognition) involves the assignment of a object (sample) with respect to known reference objects (model). In the case of tetrahedra, the model is known, and the remaining parameter space has 6 dimensions: three translational and three rotational degrees of freedom must be determined for each object. The centroids of the labeled regions from the preceding segmentation step provide a good estimate for the translational coordinates. Determining the orientation of a known object in 3D is a task for which different computational approaches exist [24, 40]. In general, an algorithm converging to the optimal sample-model transformation is needed. The orientational registration can be simplified in certain cases:

$\triangleright$ In a 2-dimensional angle space, the Hough transform is an established method to find prevalent line directions in images [31].

$\triangleright$ If particles have a anisotropic shape (principal axes), the orientation vector is equivalent to the largest eigenvector, which can be obtained via a singular value decomposition of the segmented voxels. This method is employed here for the registration of rod particles (see Sec. 5.2.3). 


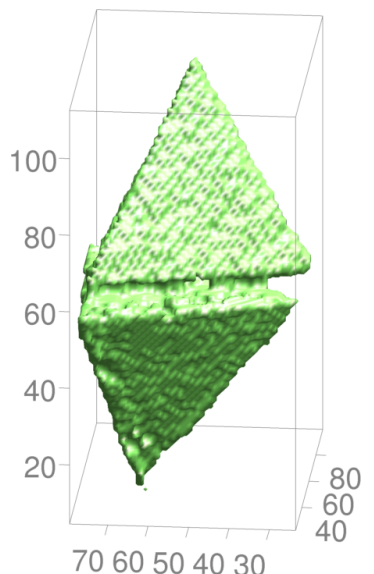

(a)

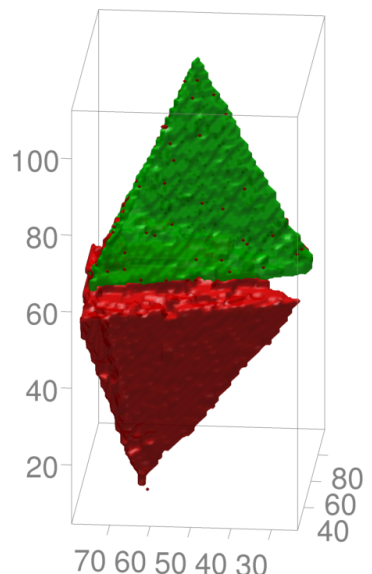

(b)

Figure 3.13: (a) Volume rendering of dublette label (b) Separated in two regions after edge enhancement

$\triangleright$ Spherical harmonics are used for pattern detection and structural analysis [40], but these are - by construction - insensitive to a specific orientation.

$\triangleright$ The point-set registration [1] is formulated as follows: Define the sample by a set of $n$ voxels $v_{n} \in \mathbb{R}^{3}$, and a model by another set $m_{n} \in \mathbb{R}^{3}$ Then find the rotation $R$ and translation $T$ (transformations matrices) that minimize the error $d=\sum_{i=1}^{n}\left\|v_{i}-T R m_{i}\right\|$. Here, $d$ is the summed distance of all points between model and image. A drawback is that the sets $v_{n}$ and $m_{n}$ must be "linked" to corresponding voxels first, that is, the sets must be ordered so that the distance between $v_{j}$ and $m_{j}$ is the shortest for all points $j$. Another complication arises if the voxel sets differ in their size, which is the typical case in experimental data.

Therefore, a two-step registration procedure for packings of the spherically symmetric platonic bodies was developed. It was implemented w.r.t. the following design specifications. The performance of the procedure regarding these points is discussed in the next Sec. 3.1.6.

1. High accuracy (alignment of particles)

2. High detection rate $(>99.80 \%)$, no false positives

3. Reasonable processing speed

4. Robustness to noisy input data (e.g. brightness variations)

5. Parallelization 
6. Extension to other particle shapes

First, a matching criterion $m \in[0 \ldots 1]$ is defined by the number of foreground (white) voxels inside a translated and rotated reference model ${ }^{2}$, normalized by the maximum possible number of enclosed voxels $n_{\max } \approx V_{\text {Tet }}$ (resp. $V_{\text {Oct }}$ ). As a pre-check, all region labels smaller than $\frac{2}{3} V_{\text {Tet }}$ voxels are immediately discarded, because they are probably oversegmented and no sufficient match is possible. (CalcOverlapOffsetAngles.m). The optimization task is then formulated as follows: For each watershed region, maximize the matching function $m(\mathbf{x})$, a w.r.t. three translational and three rotational degrees of freedom, denoted by $\mathbf{x}=(x, y, z)$ and $\mathbf{a}=(\Psi, \Theta, \Phi)$, respectively. We denote the final match value by $m^{*}$ with the final parameters $\mathbf{x}^{*}$ and $\mathbf{a} *$.

A established quasi-Newton method, the so-called Limited-Memory-BroydenFletcher-Goldfarb-Shannon-Algorithm (in short: LBFGS) is chosen for this optimization problem due to its robustness and fast convergence [41]. The following pseudocode outlines the structure of the two-step registration algorithm, which we refer to in the following sections.

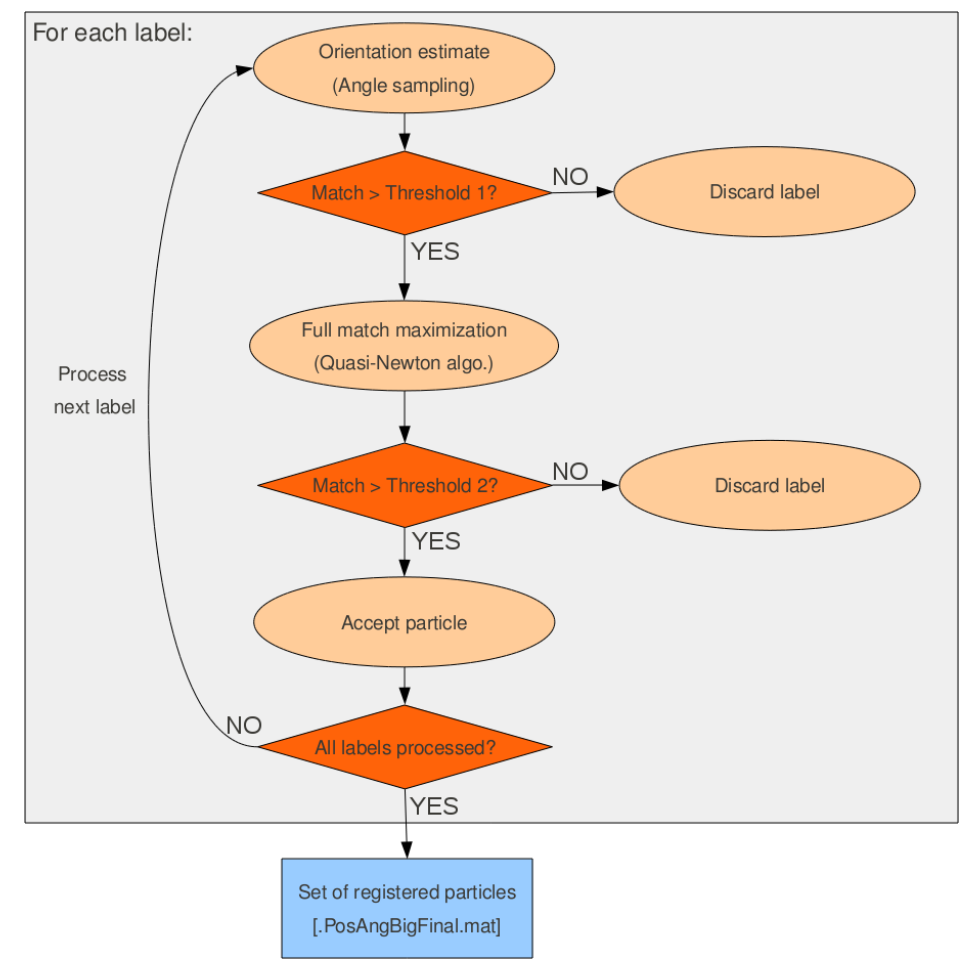

Figure 3.14: Schematic process flow of particle registration (FindOrientation_WS.m)

\footnotetext{
${ }^{2}$ Currently, reference models are restricted to convex polyhedra
} 


\section{Orientation estimation by sampling}

The first step of the object registration of particles is the choice of appropriate initial conditions, which are supposedly close to the global match optimum. This rules out the possibility of getting trapped in local minima ${ }^{3}$ and provides a substantial performance boost. To this aim, a sweep search over the full rotation space of Euler angles (SweepEulerAngles.m) is performed, and the best match is chosen as initial condition of the subsequent maximization. Since the voxel-based match computation is computationally quite expensive (one call to CalcOverlap.m takes $6 \mathrm{~ms}$ on a IntelCore 2 Duo @ 3Ghz), a speed-up-method is employed: Instead of rotating the full voxel sample, only a point-based "spine" model $S$, connecting each corner to the particle centroid, is rotated and the point-wise match is computed. In contrast to the full voxel model with $V_{\text {Tet }}=40422$ voxels, this requires only $4 * R_{o}=172$ voxels to be checked, resulting in a substantial speed-up. The centroid position $\mathbf{x}$ of the spine model is set to the centroid of the corresponding label. Fig. 3.15a shows an example of match values of the rotated spine model in the 3D-space of Euler angles, where the maxima are selected by thresholding the highest $1 \%$ and then clustered by a distance cutoff. Fig. 3.15b depicts the best-fit spine model in red, the foreground voxels in grey, and the estimated model in blue shading. The similarity to the final registration by the LBFGS algorithm (Fig.3.16) is already evident. If more than one maximum is found in angle space (depending on particle symmetry axes), all maxima are evaluated using the voxel match computation, then the angles corresponding to the best match are chosen for initialization of the LBFGS optimization.

\section{Match maximization}

LBFGS belongs to the family of Quasi-Newton methods, which are directed at iteratively finding extrema of functions using local derivatives. Newton's original method assumes the scalar function $f(\mathbf{x}): \mathbb{R}^{n} \mapsto \mathbb{R}$ to be of quadratic form, so that first and second derivatives $f^{\prime}$ and $f^{\prime \prime}$ are valid approximations in the vicinity of the extremum. In multidimensional optimization, this generalizes to the computation of the gradient $\nabla$ and the Hessian $\mathbf{H}$.

Consider the Taylor expansion of a function $f$ around the $i$ th iteration point $\mathbf{x}_{i}$ with the finite difference $\Delta \mathbf{x}$ :

$$
\begin{aligned}
f\left(\mathbf{x}_{i}+\Delta \mathbf{x}\right) & \approx f\left(\mathbf{x}_{\mathbf{i}}\right)+\nabla f\left(\mathbf{x}_{i}\right) \Delta \mathbf{x}+\frac{1}{2} \Delta \mathbf{x H} \Delta \mathbf{x} \\
\Rightarrow \nabla f\left(\mathbf{x}_{i}+\Delta \mathbf{x}\right) & =\nabla f\left(\mathbf{x}_{i}\right)+\mathbf{H} \cdot \Delta \mathbf{x}
\end{aligned}
$$

\footnotetext{
${ }^{3}$ Minimization or maximization of a function are trivially related since $\min (f(x))=$ $\max (-f(x))$.
} 


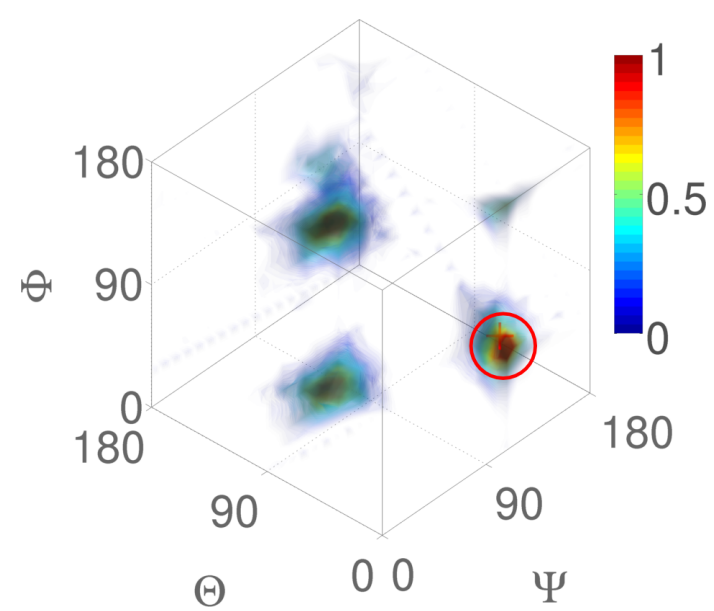

(a)

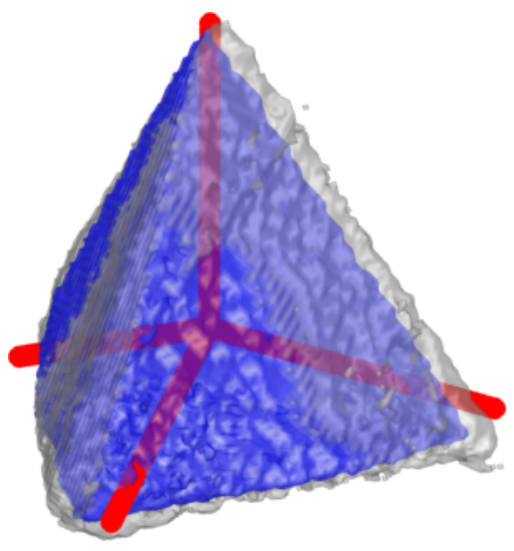

(b)

Figure 3.15: (a) Normalized match value in sampled 3D-Euler-angle space $\mathbf{a}=(\Psi, \Theta, \Phi)$ (units in degree). (b) Orientation of model corresponding to highest match of $m^{*}=0.88$, at $\mathbf{a}^{*}=\left[111^{\circ}, 9^{\circ} 77^{\circ}\right]$, see bottom right corner of Fig. (a).

The necessary condition for a extremum is that the gradient vanishes: $\nabla f\left(\mathbf{x}_{i}+\Delta \mathbf{x}\right)=0$, so the Newton step $\Delta \mathbf{x}$ is obtained from Eq. 3.3 as:

$$
\Delta \mathbf{x}=-\nabla f\left(\mathbf{x}_{i}\right) \cdot \mathbf{H}^{-1}
$$

Eq. 3.4 shows that first and second derivatives are needed to compute the step $\Delta \mathbf{x}$, which is essentially the direction towards the extremum. However, there is no analytical expression for $f$ in the present problem of match maximization, calling for numerical approximations of $\nabla$ and $\mathbf{H}^{-1}$. The gradient $\nabla$ is approximated by the finite forward difference:

$$
\nabla \approx \frac{f\left(\mathbf{x}+\Delta_{g} \mathbf{x}\right)-f(\mathbf{x})}{\Delta_{g} \mathbf{x}}
$$

Note that this finite difference $\Delta_{g} \mathbf{x}$ is not to be confused with the actual Newton step $\Delta \mathbf{x}$ !

Approximation of the inverse Hessian $\mathbf{H}^{-1}$ is performed by construction of a matrix $\mathbf{J}_{k}$, with the property $\lim _{k \rightarrow \infty} \mathbf{J}_{k}=\mathbf{H}^{-1}$, where $k$ is the number of iterative approximations. The various Quasi-Newton methods mainly differ in the way $\mathbf{J}_{k}$ is constructed, but the explicit expressions are lengthy and therefore omitted here for clarity [64]. In short, the current $\mathbf{J}_{k}$ is a function of the preceding $\mathbf{J}_{k-1}$, the current and previous gradient $\nabla_{k}$ resp. $\nabla_{k-1}$ and the current Newton step $\Delta \mathbf{x}$. In practice, the $\lim _{k \rightarrow \infty} \mathbf{J}_{k}$ is replaced by a sufficient number of iterations, this implementation uses 5 iterations. 


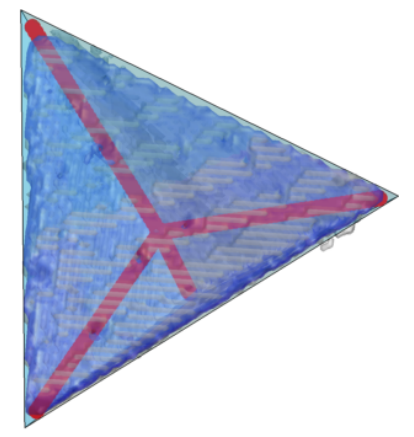

Figure 3.16: Example of registered particle after match maximization

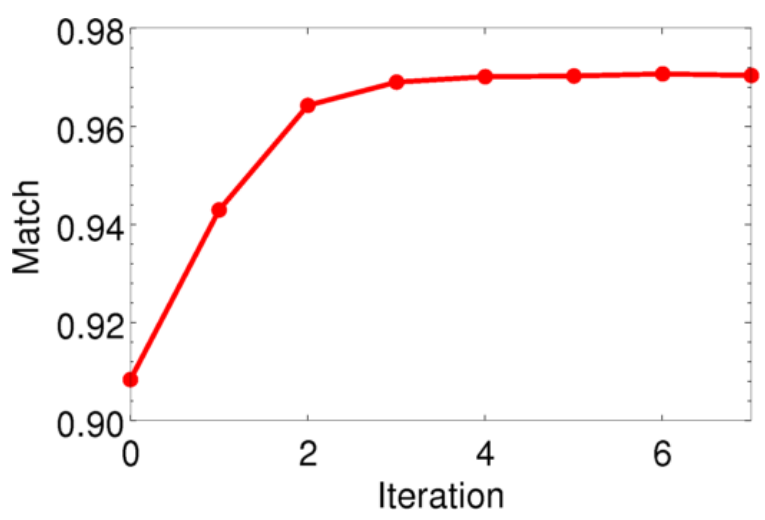

Figure 3.17

Figure 3.18: Match value $m$ vs. iteration step $i$, averaged over 30 registered particles

\section{Parameter scaling}

Convergence of the algorithm is generally reached if $\Delta x<\epsilon_{x}$ with the parameter tolerance $\epsilon_{x}=10^{-6}$ or $\Delta f(x)<\epsilon_{f}$ with the function tolerance $\epsilon_{f}=10^{-6}$. Recalling that the maximum value of the overlap function equals the tetrahedra volume of $V_{\text {tet }}=4.0422 \cdot 10^{4}$ voxels, an approximation error of less than one voxel is expected. In practice, the maximum match value is lower due to a mismatch between ideal model and physical particle geometry (see Fig. 3.19) and the specific choice of the local binarization parameters.

If a particle is successfully registered, the corresponding voxels are removed from the binary image and the final match parameters $\mathbf{x}^{*}, \mathbf{a}^{*}$ and $\mathbf{m}^{*}$ are stored (variable PosAng).

\section{Parameter scaling}

What is a good choice of the finite difference $\Delta_{g} x$ used for gradient approximation in Eq. 3.5? Initially, $\Delta_{g}=10^{-6}$ is set and the approximation of $\nabla$ is obtained from Eq. 3.5. The next gradient approximation step $\Delta_{g}$ 
is then computed as $\Delta_{g}=\|\nabla\|^{-1}$ (with the Euclidean norm $\|$ ). However, the match function $m(\mathbf{x}, \mathbf{a})$ is not continuous, but takes only discrete values (number of voxels $m \in \mathbb{N}$ ) This implies that for sufficiently small values of $\Delta_{g}$, no change in $m$ is observed and the gradient approximation $m(x, a)=m\left(x+\Delta_{g} x, a+\Delta_{g} a\right)$ is zero. Furthermore, the positions $\mathbf{x}$ have voxel units, whereas the angles a are defined in radians $([0 \ldots 2 \pi])$, so two step sizes $\Delta_{g}^{a}$ and $\Delta_{g}^{x}$ need to be defined.

Therefore, the following cutoff ranges for the angle parameter $\Delta_{g}^{a} \in[0.015 \ldots 0.25]$ and for the position parameter $\Delta_{g}^{x} \in[0.125 \ldots 2.0]$ are chosen. These values were obtained by a extensive parameter sweep, iterating towards the highest accuracy (see Sec. 3.1.6). The lower bound of the range ensures that the gradient does not vanish before actual convergence, and the upper bound limits overshooting of the parameters near extrema. Note that this intricacy occurs only because the objective function $m$ is not continuous. A possible solution is to smooth the segmented binary volume with a gaussian filter before registration. However, this filtering does not improve exact alignment, but broadens the range of the acceptance criterion $m^{*}$.

\subsubsection{Performance}

In this section, we discuss the performance of the present registration algorithm w.r.t to the desired specifications (see Sec.3.1.5).

\section{Accuracy}

A good agreement between model and images is already apparent from Fig. 3.16. Additionally, more quantitative measures are available, such as the distribution of the final match values $m^{*}$ (see Fig. 3.19), with a mean of $0.975 \pm 0.013$.

The deviation from the expected value $m^{*}=1.0$ is mostly attributable to the imperfect geometry of the physical particles. Experimentally, the corner and edge curvature of the tetrahedra was determined ${ }^{4}$ to $(150 \pm 50) \mu \mathrm{m}$. For comparison, a synthetical voxel model of a tetrahedron was created by appropriate scaling and dilation. The maximum match value of this model is $m_{\text {syn }}=0.980 \pm 0.005$, which is in good agreement with the observed upper bound of $m^{*}$ in Fig.3.19a.

A more sophisticated quality measure is provided by the analysis of the contact number scaling (CNS) model in the reconstructed particle packing; see Sec. 3.2 for physical motivation and details of the model. The number of intersections $n$ as a function of the scaled side length $a_{v}$ is given by:

$$
n\left(a_{v}\right)=Z N_{a_{\mu}, \sigma_{C N S}}+\Theta\left(a_{v}-a_{\mu}\right) \cdot s \cdot\left(a_{v}-a_{\mu}\right)
$$

\footnotetext{
${ }^{4}$ Inspection of imprints on rubber silicone with a optical microscope.
} 


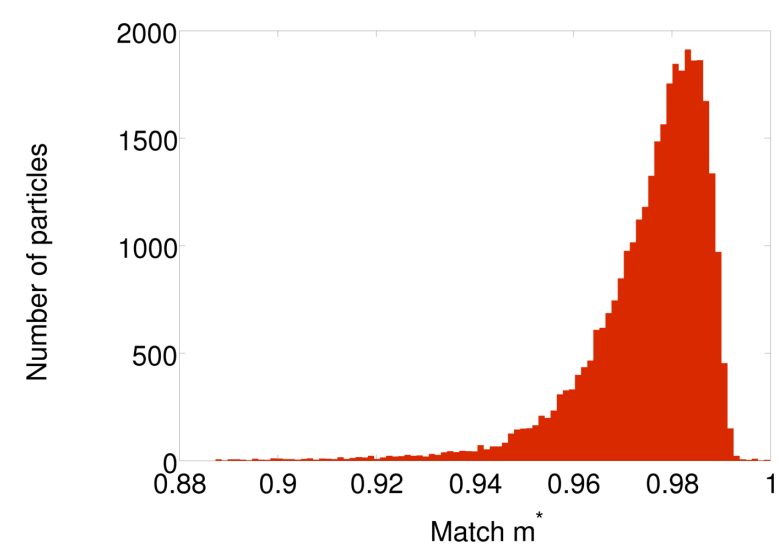

(a)

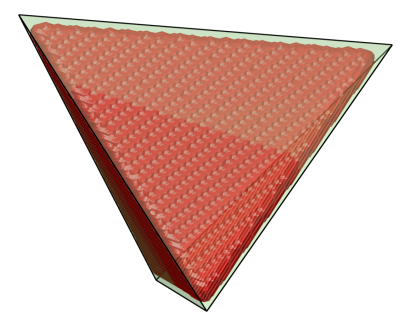

(b)

Figure 3.19: (a) Histogram of final matches $m^{*}$ ( $N=32555$ particles, data summed over 3 different samples). (b) Synthetical binary volume of tetrahedron with rounded edges, inscribed into exact tetrahedron (black line)

with the side length $a_{v}$, (average) contact number $Z$, cumulative normal distribution $N\left(a_{\mu}, \sigma_{C N S}=\right.$, and estimated (average) side length $a_{\mu}$. The parameter $\sigma_{z}$, corresponding to the width of the cumulative Gaussian, is a direct measure for the cumulated errors caused by imperfect particle shape, imaging artefacts and particle misalignment due to finite registration accuracy.

In order to investigate the accuracy of the particle detection, we run the LBFGS registration algorithm on a identical dataset with different downsampling factors. This downsampling reduces the number of voxels $n_{\text {match }}$ for the match computation by the factor mySpeedup $\in[1,2,4,8]$ : For a value of mySpeedup $=2$, only every 2 nd voxel is considered, so $n_{\text {match }}=V_{\text {tet }} / 2=40422 / 2=20211$ voxels. Downsampling by 8 is equivalent to resizing the volume by half in each dimension $\left(8=2^{3}\right)$.

\begin{tabular}{|cccc|}
\hline Downsampling & [particles $/ \mathbf{h}]$ & Accuracy $\sigma_{z}$ & Contact number $Z$ \\
\hline 1 & 1200 & .213 & 8.11 \\
2 & 1800 & .213 & 8.15 \\
4 & 2100 & .217 & 8.13 \\
8 & 2700 & .226 & 7.76 \\
\hline
\end{tabular}

Table 3.1: Effect of speedup on performance (registered particles/hour), accuracy $\left(\sigma_{C N S}\right)$, and determined contact number $Z$

Table 3.1 clearly shows that downsampling increases processing speed at the expense of accuracy. However, the best obtained $\sigma_{z}=0.213 \mathrm{~mm}$ is close to the corner curvature $(0.15 \pm 0.05) \mathrm{mm}$ and does not improve for a factor lower than 2 . This indicates that the residual error is likely to be 


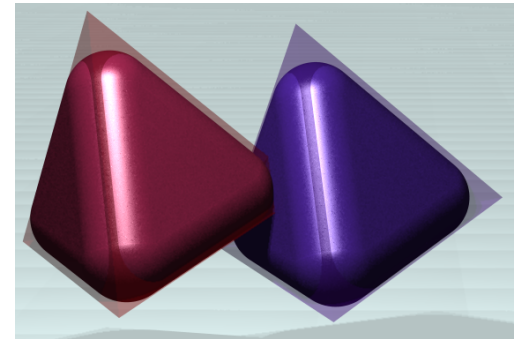

(a)

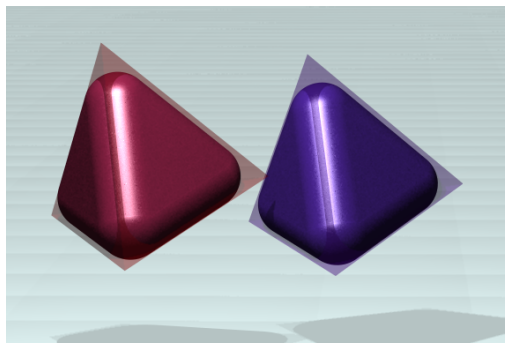

(b)

Figure 3.20: Sketch of intersection between tetrahedra: (a) Rounded particles are in contact, but models intersect (b) Reduced sidelength: No intersection

attributed to the particle geometry. This is an inherent approximation error, as already pointed out in Fig. 3.19b: The registration model assumes ideal geometric tetrahedra, whereas the physical particles have a finite radius of curvature. Fig.3.20 depicts an exemplary corner-to-face contact: the red and blue rounded tetrahedra are actually contacting, but the registered models exhibit intersection. Note that the sketch exaggerates the actual radius of curvature by a factor of 3 for illustrative purposes.

\section{Detection rate}

The detection rate $P$ is defined as the percentage of the detected particles in relation to all particles present in the sample. The current implementation reaches typically a detection rate of $P>99.80 \%$, provided the image quality is sufficiently good throughout the sample, which can be verified by visual inspection ReconstructImageOverlay.m. That is, at maximum 20 particles of 10000 are not detected (false negatives). Near the vertical and radial borders of the sample, image quality decays, therefore the ROI of analysis is restricted to a smaller cylinder. Due to the acceptance threshold for region sizes and the construction of the match criterion, false positives (matches where no particles are present) are not observed.

\section{Robustness}

The parameters in the image processing chain were tuned to yield the best results for the given datasets. Generally, for application to other experimental data, evaluating the segmentation quality in each image processing stage is recommended, and if necessary, parameters should be tuned according to the following list. Quality inspection concerns after stage I) the binary image $I_{C}$, in stage II) the label image $L$ and in stage III) the reconstructed packing (ReconstructTetOct_Overlay.m) and the width of the CNS curve. 


\section{Processing speed}

The runtime of the preprocessing and binarization stage (I) amounts only to a few minutes and is therefore negligible compared to the subsequent parts. Most processing time is spent in the segmentation part (II), particularly for the EDT (bwdist) and the WST (watershed). A complete sample of the order of $10^{3} \times 10^{3} \times 10^{3}=10^{9}$ voxels $(=1$ Gvoxel $)$ is segmented within 90 minutes, thus reaching a processing speed of 0.2 Mvoxels/s.

The registration throughput in stage (III) (at a downsampling factor mySpeedup $=2$ ) is of the order of 2000 particles/h on a IntelCore 2 Duo @ 3Ghz (see Tab. 3.1).

\section{Parallelization}

The bottleneck of segmentation in stage II) could be resolved by parallelization. There exists a highly optimized parallel solution for segmentation with a processing speed of $>10$ Mvoxels/s [94], but at the time of writing, this was not available for 3D datasets.

The registration stage is easily parallelized by serial registration (node 1 processes the subset of labels $\left[1 \ldots l_{i}\right]$, node 2 the labels $\left[l_{i}+1 \ldots 2 l_{i}\right]$ and so on), thus runtime is only limited by the number of available cluster nodes (plus negligible runtime for initialization and consolidation of the results).

\section{Parameter tuning instructions}

I Undersegmentation of $I_{C}$ ?

$\rightarrow$ Decrease window size $W$, then threshold $t$.

II Undersegmentation of $L$ ?

$\rightarrow$ Decrease h-minima threshold in steps of 1 .

III Convergence problems?

$\rightarrow$ Try to adapt the termination criteria $\epsilon_{f}, \epsilon_{x}$, range of finite difference values $\Delta_{g}^{\min }, \Delta_{g}^{\max }$. Other approach: Make the match function $m$ continuous.

For the opposite problem of oversegmentation, parameters need to be changed vice versa. The first stages are crucial for the success of the registration algorithm: starting with a good binarization (solidity of particles, low noise), both segmentation and final registration are likely to achieve a high detection rate and accuracy. 


\subsubsection{Future extensions, appendix}

\section{Octahedra and mixtures}

The watershed-based segmentation is not restricted to a certain particle shape, but works also for particle mixtures (bidisperse or polydisperse packings). This holds under the constraint that particles can be separated well enough via the EDT and the additional separation step. Fig. 3.21b shows a slice of a mixed packing, where particles tetrahedra (red) and octahedra (blue) are successfully registered and distinguished.

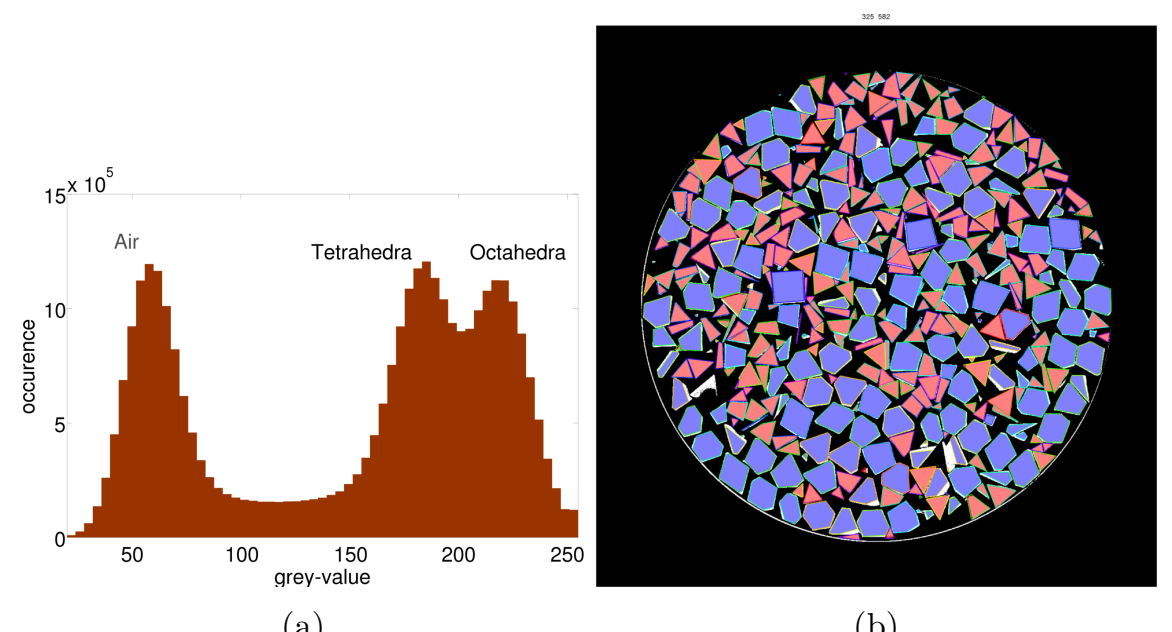

Figure 3.21: (a) Grey-value histogram of tetrahedra-octahedra mixture (b) Z-slice of mixed packing, registered tetrahedra in red and octahedra in blue.

Analysis of other mono- or multidisperse packings of polyhedra is possible by entering the model vertices into Init_Watershed.m. For any multidisperse packing, a clear distinction criterion is needed for efficient registration. In the case of tetrahedra and octahedra, this criterion is the label volume, which is on average $3.2 \cdot 10^{4}$ for tetrahedra and $1.6 \cdot 10^{5}$ for octahedra, providing a clear size threshold. The brightness variation, due to different material density, is not sufficient to classify tetrahedra or octahedra, as is evident from the overlapping peaks in Fig. 3.21a.

A side note: The side length of the current models is set to 70 voxels (=physical sidelength of $7 \mathrm{~mm}$ ). If the particle sizes are distributed in a size range, the sidelength needs to be added as additional optimization parameter for the match maximization (see MaximimizeOverlap.m), fminlbfgs $2 . m$ and CalcOverlapOffsetAngles). 


\subsection{Determination of contact numbers}

The geometric representation of the tetrahedra particles is a prerequisite for examining packing structures, contact numbers and Voronoi volumes. To begin with, the problem of contact number counting is addressed.

Inter-particle contacts cannot be determined directly from tomographic data because the result is affected by particle polydispersity, imaging artefacts and the finite accuracy of particle detection. However, Aste et al. [3] introduced a method that can provide a physically justified average contact number for spheres of diameter $d$, under the assumption that experimental errors are distributed gaussian. It defines the number of neighboring particles $n(r)$, which includes all particles with a center to center distance smaller or equal to $r$. In the case $r<d, n(r)$ can be described by multiplying the average contact number $Z$ with the cumulative normal distribution $N \mu, \sigma(r)$ :

$$
N_{\mu, \sigma}(r)=\frac{1}{\sigma \sqrt{2 \pi}} \int_{-\infty}^{r} \exp \left(-\frac{(x-\mu)^{2}}{2 \sigma^{2}}\right) \mathrm{dx}
$$

The mean $\mu$ provides an estimate for the bead diameter $d$, and the variance $\sigma$ corresponds corresponds to the combined effects from polydispersity and uncertainty of tomography and particle detection.

For $r>d$, there is an additional term to $Z N_{\mu, \sigma}(r)$ which describes the growing contribution from spurious contacts, near neighbors which are close but not in contact. The latter part can be approximated (in lack of deeper knowledge) by a linear function $f_{\text {lin }}(r)=m \cdot(r-d)$ with slope $m$ combined with the Heaviside step function $\Theta(r-d)$, leading to the complete model for $n(r)$ :

$$
n(r)=Z \cdot N_{d, \sigma}+\Theta(r-d) \cdot f_{l i n}
$$

Figure 3.22 shows an illustration of the model for spheres with a contact number $Z=6$.

We transfer the model to tetrahedra packings as follows: Instead of counting neighbors within a distance $r$, particles are scaled by a "virtual" side length $a_{v}$ and the number of intersections $n\left(a_{v}\right)$ is counted ${ }^{5}$, as shown in Eq. 3.9:

$$
n\left(a_{v}\right)=Z N_{a_{\mu}, \sigma}+\Theta\left(a_{v}-a_{\mu}\right) \cdot m \cdot\left(a_{v}-a_{\mu}\right)
$$

with the estimated sidelength $a_{\mu}$.

This model is termed "contact number scaling function", in short CNS function, because it maps the particles [72]

\footnotetext{
${ }^{5}$ A substantial speedup of the analysis is reached by using neighbor cell lists and testing only particles within a diameter of the circumsphere.
} 


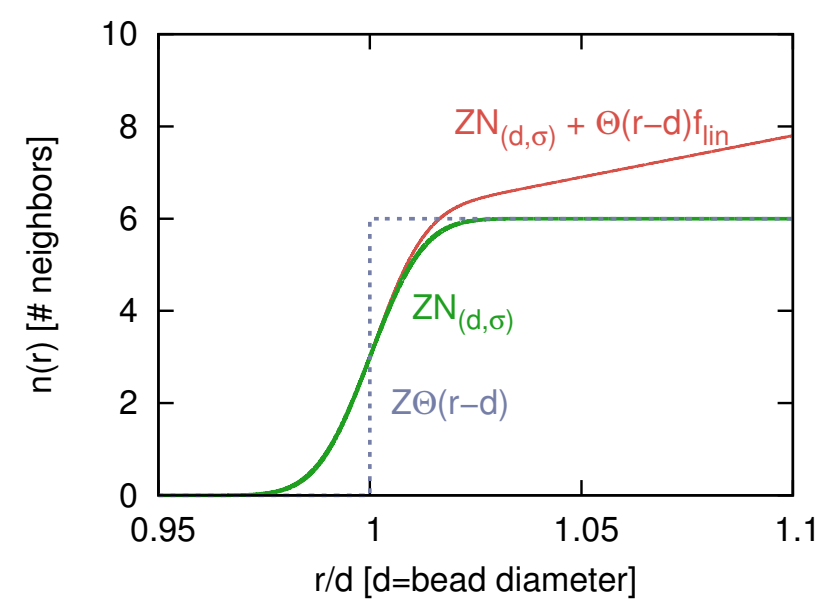

Figure 3.22: Model for the number of apparent neighbors $n$ as a function of the center-to-center-distance $r$ in a packing of spheres with diameter $d$ and contact number $Z=6$. The resulting contact number can be read off after deconvoluting the function by the scaled cumulative normal function $N_{d, \sigma}$.

Exemplary contact curves for loose and dense packings are presented in Fig. 3.23. Firstly, the side length $a_{\mu}$, the contact number $Z$, the variance $\sigma$ and the linear slope $m$ are fitted to the data, using the gnuplot implementation of the non-linear least-squares Levenberg-Marquardt algorithm. Since particles and experimental setup are identical for all 25 samples, we determine the average sidelength $\bar{a}_{\mu}=(7.02 \pm 0.03) \mathrm{mm}$ and the average variance $\bar{\sigma}=(0.206 \pm 0.015) \mathrm{mm}$ from all fits ( \pm errors are standard deviations). In a second step, these parameters are fixed, leaving $Z$ and $m$ as the only free parameters.

\subsubsection{Estimation of error bars}

The error estimate $\Delta Z$ of the contact number $Z$ can be calculated by the propagation of error principle (Gauss'sche Fehlerfortpflanzung). $Z$ is a function of the estimated sidelength $\bar{a}_{\mu}$ and the distribution width $\bar{\sigma}$, with the variances $\Delta \bar{a}_{\mu}$ and $\Delta \bar{\sigma}$. We denote the errors of the mean values with $\tilde{a}_{\mu}$ and $\tilde{\sigma}$. The error of the mean value, $\tilde{a}_{\mu}$, is computed by $t_{s} \cdot \frac{\Delta \bar{a}_{\mu}}{\sqrt{n}}$ (with the Student-t-distribution factor $t_{s}=2.13$ according to $n=20$ samples and $95 \%$ confidence), respectively for $\tilde{\sigma}$.

The error of $Z$ is then computed as follows:

$$
\Delta Z=\sqrt{\left(\frac{\partial Z}{\partial \bar{a}_{\mu}} \cdot \tilde{a}_{\mu}\right)^{2}+\left(\frac{\partial Z}{\partial \bar{\sigma}} \cdot \tilde{\sigma}\right)^{2}}
$$

where the partial derivates are approximated by the difference quotient 


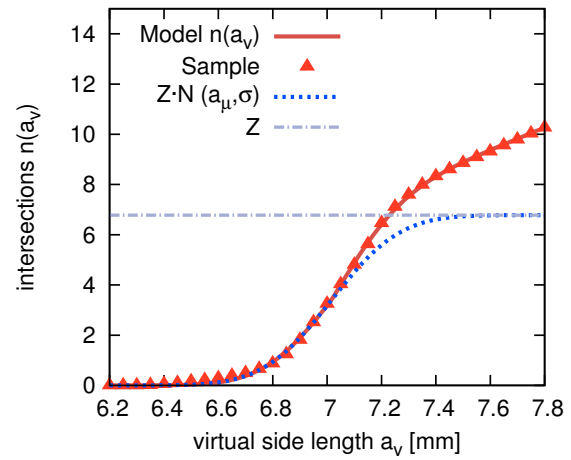

(a) $\Phi=0.470, Z=7.1$, sample: initial preparation

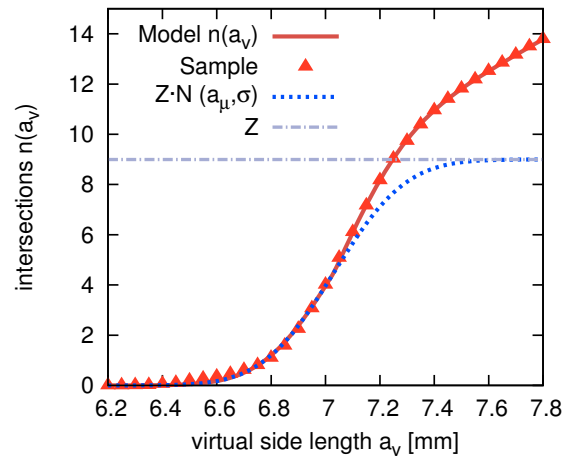

(b) $\Phi=0.600, Z=8.8$, sample:

$\operatorname{RAMP}_{\Gamma=0.25}$

Figure 3.23: Contact number analysis of tetrahedra packings for (a) loose and (b) dense packing. The data from the samples (red triangles) is well approximated by the model $n\left(a_{v}\right)$ (solid red line) using only $Z$ and $m$ as fit parameters. Deconvolution into the cumulative gauss $N\left(a_{\mu}, \sigma\right)$ (dotted blue line) and the contribution $f_{\text {lin }}$ allows to read off the contact number $Z$ (dash-dotted grey line).

approximation:

$$
\frac{\partial Z}{\partial \bar{\sigma}} \approx \frac{Z(\bar{\sigma})-Z(\bar{\sigma}+\Delta \bar{\sigma})}{\Delta \bar{\sigma}} .
$$

and the analogous expression for $\bar{\sigma}_{\mu}$.

\subsection{From contacts to constraints}

Four different contact geometries are observed in tetrahedra packings, as table 3.2 illustrates: face-face $(\mathrm{FF})$ contacts, edge-face $(\mathrm{EF})$ contacts and the point contacting configurations of vertex-face (VF) and edge-edge (EE). Vertex-Vertex or Vertex-Edge contacts are not observed in practice.

Mechanical stability of a granulate packing implies force balance at the contacts - that means, the number of mechanical constraints $C$ imposed by a contact must be equal (isostatic) or larger (hyperstatic) than the degrees of freedom (DOF) per particle. For frictionless spheres having only single point contacts, one obtains the isostatic contact number $Z_{\text {iso }}=2 \cdot \mathrm{DOF}=6$. However, this does not hold for tetrahedra packings, because the number of mechanical constraints fixed by a contact depends on the specific contact geometry. Evaluating the number of constraints per contact type is best visualized by keeping one tetrahedron as "fixed", and considering the restricted DOF of a second contacting "probe" tetrahedron. In the presence of friction, all contact types block three translational degrees of thie probe tetrahedron by introducing one normal and two tangential forces. That 
means, sliding of particles past each other is not allowed without external force. The contact types do however differ in the amount of blocked rotational degrees: A frictional face-to-face contacts blocks 3 rotations: one around the surface normal (by friction) and 2 rotations around the two axis standing perpendicular on the surface normal (by non-overlap). An EF contact blocks only one rotations perpendicular to the surface normal. Lastly, the point contacts VF and EE do not block any rotation at all. As these constraints are always shared between the "fixed" and the "probe" tetrahedron, we obtain the constraint multipliers $C_{F F}=3.0, C_{E F}=2.5$, and $C_{V F}=C_{E E}=1.5$. Multiplying these numbers with the according type-specific contact numbers in a specific configuration gives the number of constraints per particle $C$.

In previous work, the generalized isostastic number was given as $C=12$ $[34,37]$, but this refers to a particle pair. For consistency with the contact number $Z$, defined per particle, the same notion should be adopted for the constraint number.

\begin{tabular}{lllll}
\hline Type & face-face & edge-face & vertex-face & edge-edge \\
\hline
\end{tabular}

Table 3.2: From top to bottom: Contact geometries (real classifications from a experimental sample); constrained DOF; constraint multipliers $C_{F F}, C_{E F}, C_{V F}$, $C_{E E}$;

\subsubsection{Analysis of the contact geometry}

In order to determine local contact geometries, all tetrahedra of one sample are scaled to the "contacting" side length $a_{c}$, so that the corresponding $n\left(a_{c}\right)=Z$ is consistent (see Fig. 3.24). Then the contact geometry of all particles with their intersecting neighbors is analysed. These intersections are not physical, but appear due to the corner curvature and the larger side length $a_{c}$.

The classification algorithm is based on a iterative procedure: First the face-face angle $\alpha_{F F}$ between normals of adjacent faces is computed; this is 


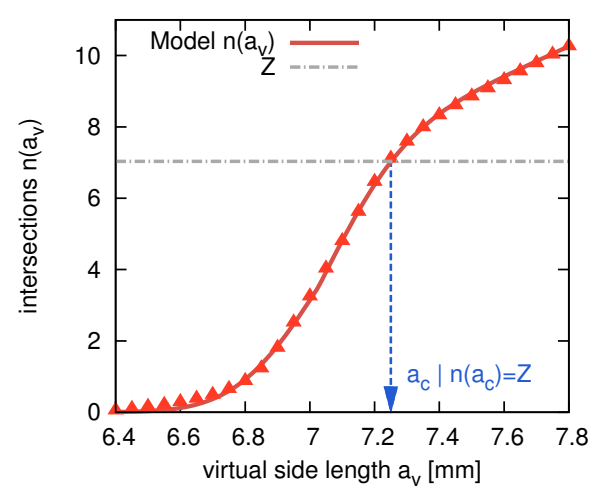

Figure 3.24: The side length $a_{c}$ with $n\left(a_{c}\right)=Z$ is given by the intersection of the model $n\left(a_{v}\right)$ with $Z$.

$180^{\circ}$ at perfect alignment. Because of the finite resolution of tomography and particle detection, a contact is classified as $\mathrm{FF}$ if $\alpha_{F F}>\alpha_{F F}^{\min }$. The procedure for determination of $\alpha_{F F}^{\min }$ is outlined in the following section.

In the absence of a calibration method for edge-face contacts, the FF threshold also applies for the classification of edge-face (EF) contacts. That means, a contact is classified as EF if the edge is collinear with the face: $\alpha_{E F}<180^{\circ}-\alpha_{F F}^{\min }$.

Finally, the configuration is checked for vertex-face contacts (VF) by evaluting if one vertex of a tetrahedron is inside the contacting neighbor ${ }^{6}$. Any remaining contacts are then attributed to edge-edge contacts. This procedure was verified by constructing test cases and additional visual inspection of the resulting classification.

\subsubsection{Threshold choice for FF and EF contacts}

It has been shown[80] that an arbitrary choice of $\alpha_{F F}^{\min }$ can lead to a physically infeasible constraint number[34], therefore the threshold must be chosen carefully. To this aim, five different samples containing only face-to-face contacts ("Face-to-Face sample") are prepared by glueing one tetrahedron corner-down to a plate and adding another tetrahedron face-down on the top face of the first. A tomographic reconstructions of one of the samples is shown in Fig. 3.25a and the according cumulative distribution of $\alpha_{F F}$ from all samples (containing 90 tetrahedra pairs) is presented in Fig. 3.25b.

A cumulative normal function as in Eq. 3.7 with mean $\mu=1.8^{\circ}$ and variance $\sigma=1.3^{\circ}$ is a good model for the distribution of the face-face angle $\alpha_{F F}$.

\footnotetext{
${ }^{6}$ This needs to be checked vice versa: Is any vertex of A inside B or any vertex of B inside $\mathrm{A}$ ?
} 


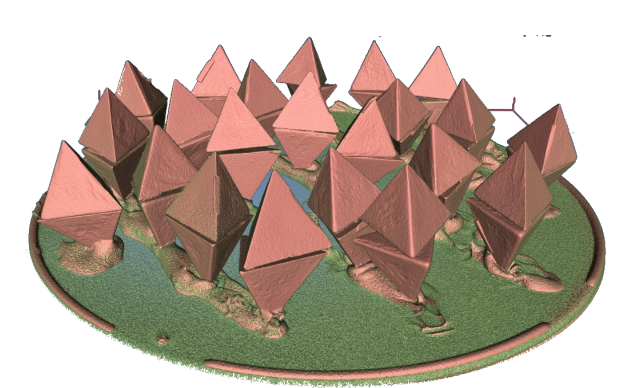

(a)

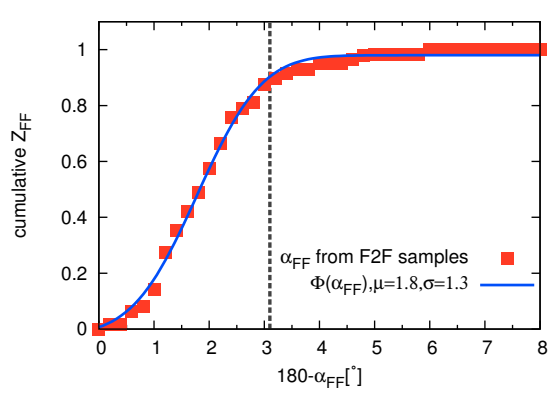

(b)

Figure 3.25: Calibration sample containing only face-face contacts for determination of threshold $\alpha_{F F}^{\min }$. (a) Tomographic reconstruction of the calibration sample (b) Cumulative distribution of the number of face-face contacts $Z_{F F}$ per tetrahedron. Chosen threshold of $\mu+1 \sigma$ is marked by the dotted grey line.

According to the face-face calibration sample, the threshold $\alpha_{F F}^{\min }=\mu-\sigma=176.9^{\circ}$ is selected.

\subsection{Analysis of Voronoi volumes}

The distribution of Voronoi volumes plays a central role for the statistical description of granular matter (e.g. employed in tomography of sphere packings [2]). In order to obtain the set Voronoi diagram of the packings [71], the so-called feature transformation (function bwdist) is utilized here on the image: This transformation assigns each voxel in the tomographic volume to the label of the nearest particle, and is therefore a discretized version of the set Voronoi diagram. An advantage of this version is that it can process arbitrary shapes of the regions or particle with a accuracy of 1 voxel. An illustration of the resulting decomposition is presented in Fig. 3.26.

Results on tetrahedra packings are discussed in detail in 4.4.1. 


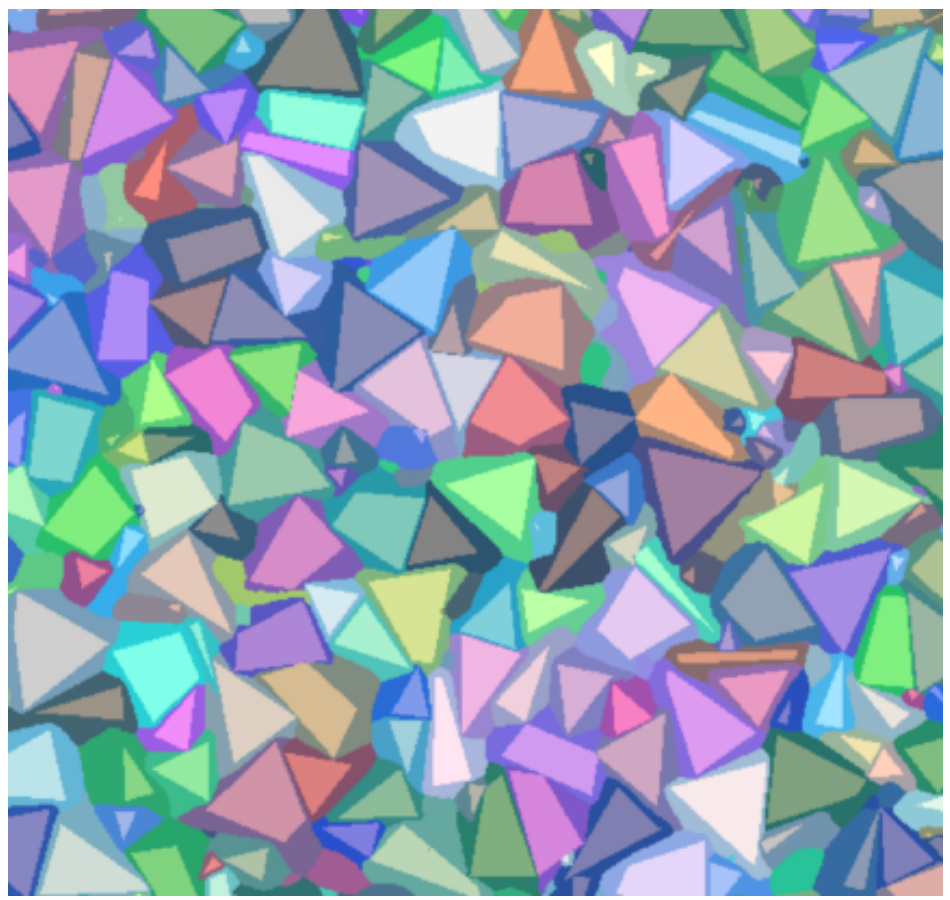

Figure 3.26: Exemplary part of z-slice (sample: initial, $\Phi=0.470$ ), showing coloured Voronoi cells and particles overlayed in half-transparent view. 


\section{Chapter 4}

\section{Results and Discussion}

This chapter presents and discusses the geometric structure of disordered tetrahedra packings, addressing the following central questions:

$\triangleright$ Which global packing fractions are accessible by different preparation protocols? (Sec. 4.1)

$\triangleright$ What is the contribution of different contact types to mechanical stability? (Sec. 4.2)

$\triangleright$ What are appropriate metrics to characterize order in tetrahedra packings? (Sec. 4.3)

$\triangleright$ What is the relation between local packing densities and local geometric structures? (Sec. 4.4)

To begin with, the influence of different preparation techniques on the global packing fraction is presented. This includes also the spatial distribution of local packing fraction in order to assess the packing homogeneity.

Mechanical stability in the context of the Jamming paradigm is characterized by global contact numbers and contact types (face-face, edge-face and point contacts).

The translational order is measured globally by the pair correlation function, and the orientational order via the face correlation function. Deeper insight about local configurations at different densities is then obtained by mapping local contact geometries to local packing fraction via their Voronoi volumes. Both the observed contact geometries and the Voronoi volumes suggest that locally dense arrangements of tetrahedra involve many face-face contacts. In this context, the shape of clusters with low Voronoi volume and the distribution of face-face contacting clusters are investigated further.

Finally, our experiments are compared to previous work, including other experiments on tetrahedral particles [34, 96, 6], Monte-Carlo (MC) simulations $[86,37,26]$ and distinct element method (DEM) simulations [62, 61]. 


\subsection{Protocol dependency of packing fractions}

The first section discusses the accessible range of packing fractions $\Phi$ for the different preparation protocols. This comprises the loose preparation, the gentle pulse protocol $\mathrm{TAP}_{\Gamma=2}$, the RAMP (annealing) protocol, and the continuous vibration (VIB). In addition, the influence of higher tapping intensity is analysed for protocol $\mathrm{TAP}_{\Gamma=3 \ldots 7}$.

\subsubsection{Initial preparation}

The "initial" preparation is designed to find the loosest mechanically stable tetrahedra packings in the present setup, and moreover, to create a reproducible initial state for the subsequent compaction experiments (TAP and RAMP). Using the manual pouring + relaxation technique as presented in Sec. 2.2.3, packing fractions in the range of $\Phi=0.469-0.477$ are achieved. Even looser packings could be supposedly created by air- or water-fluidized beds at slow sedimentation speed, which have been employed for creating the loosest known sphere packing with $\Phi_{R L P}=0.550 \pm 0.001$ [35]. This technique is difficult to adapt for tomographic analysis, because at the onset of mechanical stability, particle arrangements during imaging are very likely, causing erroneous regions in the reconstructed volume. As preliminary experiments with wetted tetrahedra show, water (with or without wetting agent) creates liquid bridges between particles, hampering the particle detection.

The lowest packing fractions using the "initial" protocol match the results from Baker and Kudrolli [6], who found $\Phi_{\min }=0.48 \pm 0.02$. Thus the current limit of mechanically stable tetrahedra packings is supposedly near $\Phi_{\min } \approx 0.47$, yet particles with higher friction are expected to reach even lower densities.

\subsubsection{Protocol TAP $(\Gamma=2)$}

As Fig. 4.1a and Fig. 4.1b show, successive gentle taps with intensity $\Gamma=2$ compact the pile slowly, and $\Phi$ reaches a steady state after approximately $1-5 \cdot 10^{4}$ taps. This behaviour is consistent with a stretched exponential, the so-called KWW (Kohlrausch-Williams-Watts) function, which is widely employed to describe the relaxation of out-of-equilibrium systems like glasses (see e.g. [20]):

$$
\Phi(t)=\Phi_{\infty}-\left(\Phi_{\infty}-\Phi_{0}\right) \exp \left(-(t / \tau)^{\beta}\right)
$$

with the initial packing fraction $\Phi_{0}$, the steady-state $\Phi_{\infty}$, the characteristic time $\tau$ and the fit parameter $\beta$. The final parameters are determined by averaging from three such compaction experiments: $\Phi_{0}=0.459 \pm 0.011$ $, \Phi_{\infty}=0.595 \pm 0.004, \beta=0.34 \pm 0.06$ and $\tau=600 \pm 60$. The values of $\Phi_{\infty}$ and $\tau$ are sketched as dotted lines in Fig. 4.1a. The packing fractions from 
height measurements are corrected by the linear calibration from $2.4 \mathrm{~b}$. Note that each sample in Fig. 4.1b is independently created by starting from a initial packing, then applying the defined number of taps and recording a tomography at the end.

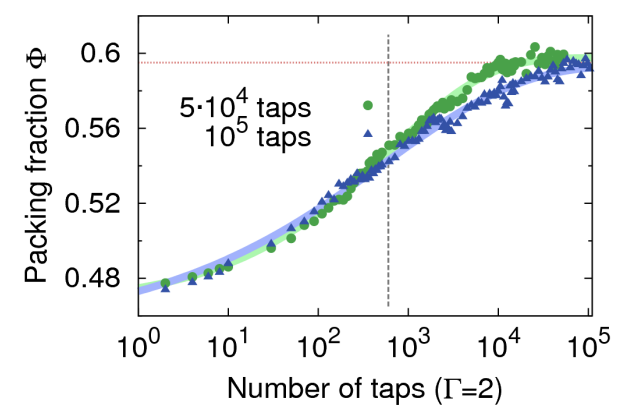

(a)

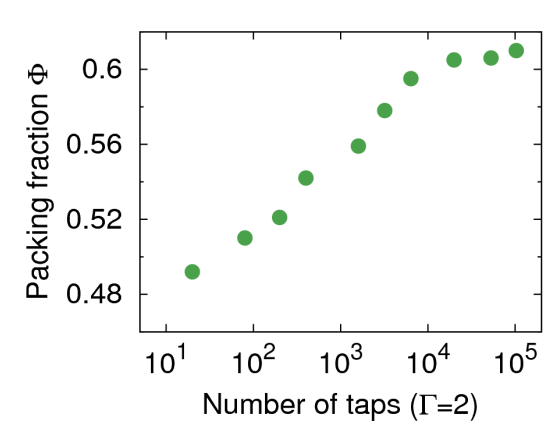

(b)

Figure 4.1: (a) Tapping at $\Gamma=2$ compacts the sample until it reaches a steady state packing fraction $\Phi_{\infty}$ between $10^{4}$ and $10^{5}$ taps, two independent runs are shown, lines are fits to Eq. 4.1. $\Phi$ approximated by laser height measurement as described in Sec. 2.2.2. Vertical dotted line corresponds to $\tau=600$, horizontal dotted line to $\Phi_{\infty}=0.595$. (b) Packing fractions of 13 samples after a number of applied taps, $\Phi$ computed from tomographic reconstruction in central ROI. Packing fractions are slightly higher than in the full volume due to lower density at the cylinder borders.

Confinement effects play an important role for the compaction dynamics and the range of attainable packing fractions. A selection of compaction experiments is listed for comparison in the following Tab. 4.1. Consider the compaction experiments by Nowak et al. [55]: These were performed in a long narrow tube with a cross-section of only 10 particle diameters and a vertical aspect ratio of approx. 50:1. This geometry prevents convection rolls, and allows a local measurement of the packing fraction, but induces crystallization from the boundary layers, leading to values of $\Phi$ clearly above the random close packing limit. A different relaxation law (the "Chicago"fit) was proposed for this setup, where the fit parameters where found to depend only on $\Gamma$. In the following sections, the protocol dependency of $\Phi_{\infty}$, is discussed with respect to the RAMP and TAP $_{\Gamma=3 \ldots 7}$ protocols.

\subsubsection{Annealing procedure (RAMP)}

The evolution of $\Phi_{\infty}$ is depicted in Fig. 4.2, and comprises the following regimes: The loose packing irreversibly compacts when $\Gamma$ is increased from 0.25 to 1.5 , but dilates again when the acceleration is further ramped to $\Gamma=5$, leading to $\Phi_{\infty} \approx 0.56$. Subsequently, decreasing the tap intensity down to $\Gamma=0.25$ ends up at $\Phi_{\infty} \approx 0.61$ (sample $\mathrm{RAMP}_{\Gamma=0.25}$ ). Increasing the intensity again to $\Gamma=5.0$ demonstrates that no hysteresis is observed 


\begin{tabular}{llllc}
\hline Ref. & $\begin{array}{l}\text { Bead } \\
\text { diameter } \\
d_{\text {particle }}\end{array}$ & $d_{\text {cylinder }} / d_{\text {particle }}$ & $h_{\text {cylinder }} / d_{\text {cylinder }} \Phi$ \\
\hline \hline $\begin{array}{l}\text { Nowak[55] } \\
\text { (Chicago) }\end{array}$ & $2 \mathrm{~mm}$ & 9.4 & 53 & $.59-.66$ \\
$\begin{array}{l}\text { Richard[68] } \\
\text { (Rennes) }\end{array}$ & $0.2-0.4 \mathrm{~mm}$ & 27 & 10 & $.57-.64$ \\
$\begin{array}{l}\text { Ribiere[66] } \\
\text { (Rennes) }\end{array}$ & $1 \mathrm{~mm}$ & 100 & 1 & $.58-.63$ \\
\hline $\begin{array}{l}\text { Tetrahedra } \\
\text { (this work) }\end{array}$ & $7 \mathrm{~mm}^{*}$ & $15-18$ & $1-1.5$ & $.47-.62$ \\
\hline
\end{tabular}

Table 4.1: Survey of compaction experiments on bead packings: particle diameter $d_{\text {particle, }}$, ratio of cylinder diameter (cross-section) to grain diameter $d_{\text {cylinder }} / d_{\text {particle }}$; height-to-diameter aspect ratio of cylinder $h_{\text {cylinder }} / d_{\text {cylinder }} ;$ all numbers rounded to two-digit precision. ${ }^{*}$ Sidelength of tetrahedra

in the reversible branch. Only the end points of the reversible branch, i.e. $\operatorname{RAMP}_{\Gamma=0.25}$ and $\mathrm{RAMP}_{\Gamma=5}$ are analysed tomographically. The accurate packing fractions in the ROI are then used for a new calibration of the height measurement, and packing fractions in between are linearly interpolated.

Ribiere et al. have ascribed the initial irreversible compaction - also observed in the RAMP experiments by Nowak et. al - to a transient effect $[66,67]$ : At low $\Gamma$, the compaction dynamics are exceedingly slow and $\tau$ diverges, but the packing can still reach a stationary state, if it is tapped long enough. In this case, only the "reversible branch" is recovered, which indicates that the stationary state with $\Phi_{\infty}$ does not depend on history, but only on the preparation protocol. The packings prepared by Ribere et. al are supposedly in a dynamical equilibrium between compaction and convection, with the steady state packing fraction $\Phi_{\infty}$ depending only on $\Gamma$. In the following Section, the question of protocol and history dependency is tackled by establishing a link between the TAP and RAMP protocol.

\subsubsection{Protocol dependency of $\Phi_{\infty}$}

The development of the reversible branch in Fig. 4.2 raises a question: Is it possible to reach the steady states at $\mathrm{RAMP}_{\Gamma=1 \ldots 5}$ directly, without the preceding annealing history? To this aim, starting from a initially loose packing, $10^{4}$ taps for a range of intensities $\Gamma$ are applied, and the obtained packing fraction is compared to the steady state of the RAMP protocol. Fig. 4.3 presents compaction curves for $\Gamma=(1 \ldots 7)$; each sample is tomographically recorded after $10^{4}$ taps. For tapping at $\Gamma=1$, the exceedingly slow compaction dynamics are apparent, and the packing is far from reaching the steady state (top line in Fig. 4.3). For higher $\Gamma$, the applied number 


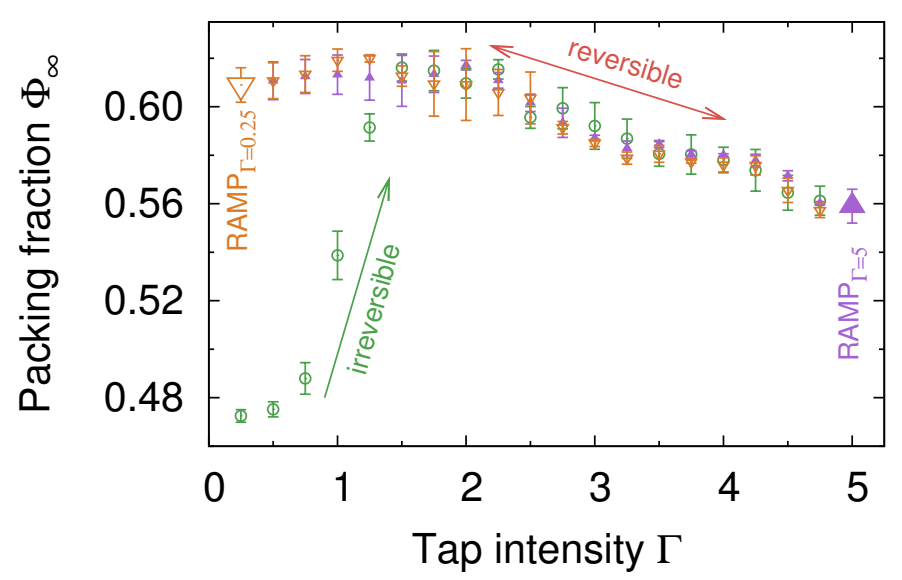

Figure 4.2: Protocol RAMP: At each acceleration step, the packing is tapped $10^{4}$ times. Open circles (green): initial, irreversible compaction. Open downward triangles (orange): decreasing acceleration. Filled upward triangles (violet): increasing acceleration on the reversible branch. Packings at points $\operatorname{RAMP}_{\Gamma=0.25}$ and $\mathrm{RAMP}_{\Gamma=5}$ are analyzed via tomography. All error bars are averaged over 4 independent runs.

of $10^{4}$ taps seems to be barely sufficient to reach a steady state $\Phi_{\infty}$ close to the respective RAMP states. Given the variations between samples, as depicted in Fig. 4.4, the final packing fractions do not depend on the annealing history (RAMP), but only on the intensity $\Gamma$. However, strong fluctuations are observed, and more ensemble averages are needed for a conclusive interpretation.

In addition to that, an interesting feature emerges: $\Phi_{\infty}$ is inversely related to $\Gamma$. MOreover, if $\Gamma \geq 3$, the density reaches an intermediate maximum, occuring earlier for higher $\Gamma$. For longer tapping series up to $10^{5}$ taps, $\Phi$ fluctuates and does not recover the intermediate maximum anymore. Up to now, the physical reason behind the decompaction after 100-1000 taps is still unclear. The slight increase of $\mathrm{TAP}_{\Gamma=5}$ or $\mathrm{TAP}_{\Gamma=7}$ towards the end could point to a long-term convection cycle. Another possibility is that static charging between the plastic tetrahedra or the plexiglas container sets in at higher shaking amplitudes, which would induce repulsive forces and thus lower the packing fraction. To investigate the latter effect, a constant flow of ionized air was directed into the cylinder during preparation, but the feature was repeatably observed. Fig. 4.4 shows the compaction of three independent samples using protocol $T A P_{\Gamma=5}$, with and without ionized air flow. All samples share the feature of the intermediate maximum and a following decompaction, but have a low overall repeatability. 


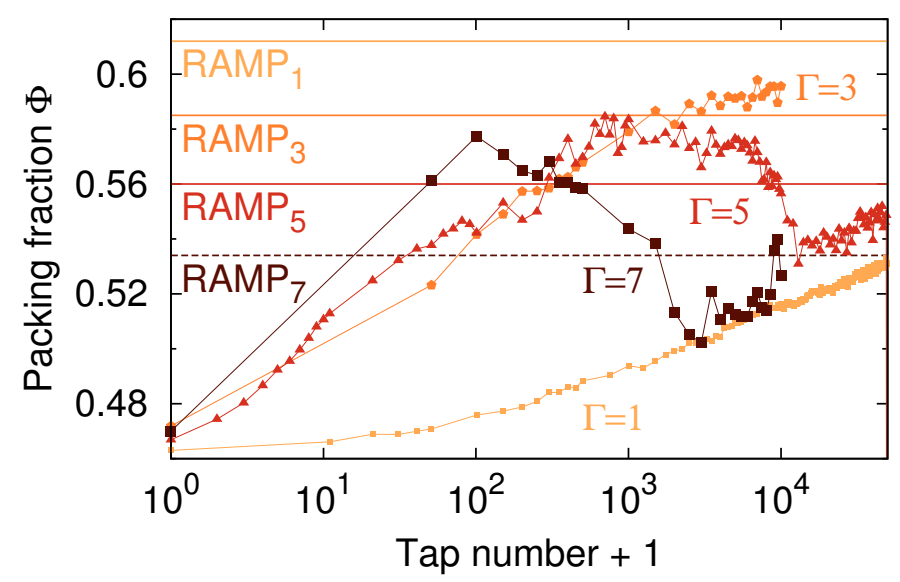

Figure 4.3: Evolution of packing fraction for different acceleration intensity $\Gamma$, tapped $10^{4}-5 \cdot 10^{4}$ times. (color map: $\Gamma$ increasing from bright to dark). The lines correspond to the steady states of the RAMP protocol, the non-existent $\mathrm{RAMP}_{\Gamma=7}$ state (dotted line) is extrapolated from the higher packing fractions.

\subsubsection{Vibration protocol (VIB)}

Among all preparation protocols, slow manual pouring of particles under constant vibration of $100 \mathrm{~Hz}$ and $\Gamma=5$ (VIB) yields the densest packings with $\Phi>0.62$. Fig. 4.5 shows a layer near the bottom of a VIB sample (binary image), where possible pentamer arrangements are marked with circles, roughly connecting the involved centroids. Some pentamers seem to form a interconnected network, but these interpretations from a single volume slice are not conclusive. Due to the manual pouring, samples created with the VIB protocol are generally difficult to reproduce.

\subsubsection{Comparison to previous work}

All in all, the range of mechanically stable packings between $\Phi=0.469$ and 0.622 is in accordance with the range of $\Phi=0.48$ to 0.64 found previously for tetrahedral dice and ceramic tetrahedra particles, prepared by shaking and by water-fluidization[6]. However, it differs widely from Jaoshvili's or Zhao's claimed values of $\Phi \approx 0.72[34,96]$

What are possible explanations for these significant differences in packing fraction? One possibility is the shape difference: The corners of tetrahedral dice are less acute than our injection-moulded particles, as Fig. 2.1a already illustrated. However, shape fails to conclusively explain the differences between packing fractions of tetrahedral dice and our particles. Following the numerical results, the larger corner radius of the dice as used by Jaoshvili[34] or Zhao[96] should cause a lower packing fraction. Therefore, a more likely explanation for the relatively low $\Phi$ found in the present work is the high friction coefficient of $\mu \approx 0.8$ compared to $\mu \approx 0.2$ of the dice. Higher fric- 


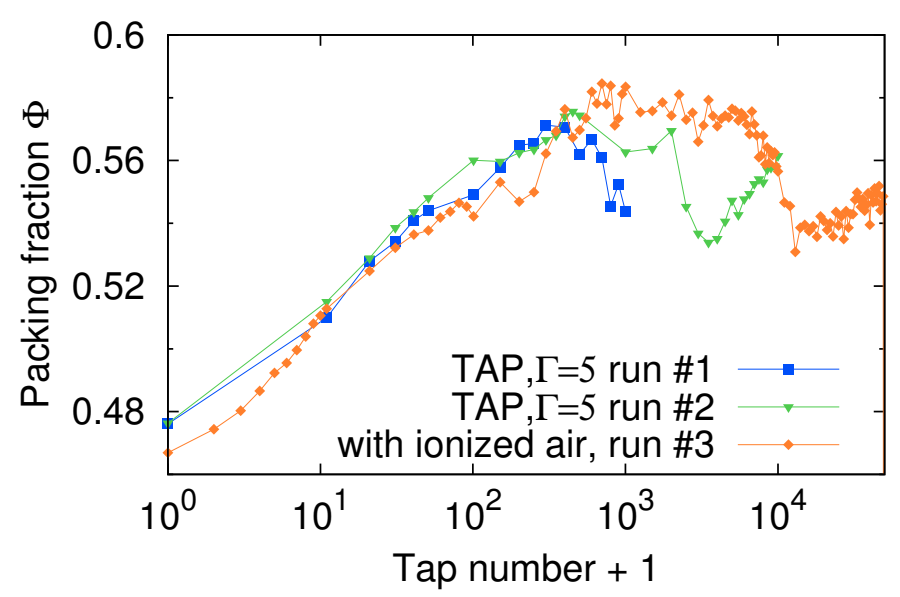

Figure 4.4: Variation of compaction behaviour between different compaction runs: Tapping at $\Gamma=5$ (run \#1 and \#2), run \#3 with continuous flow of ionized air directed inside the cylinder to eliminate static charging.

tion causes the packings to become jammed earlier, because less contacts are needed for mechanical stability [77].

Please refer to Tab. B.1 in the Appendix for a comprehensive overview of the differences between Ref. [34] and this study.

\subsubsection{Spatial distribution of $\phi_{\text {local }}$}

How homogeneous are the global packing densities w.r.t. different preparation techniques? The local packing fractions $\phi_{\text {local }}$, as computed from the particles' Voronoi volumes $V$ by $\phi_{\text {local }}=V_{\text {Tet }} / V$, allow an analysis of the spatial distribution. Fig. 4.6a shows vertical density profiles from a loose (0 taps) to a dense sample with 50000 taps. Judging from the vertical distribution for 1600 and 50000 taps, relative compaction is more prominent near the bottom of the container, probably due to higher pressure. The radial averaging is performed over constant-area rings, thus the binning width decreases with radial distance (similar to the radial binarization in Sec.3.1.3). One observes that the initial, loose packings have a lower density near the cylinder walls, due to the gentle outwards relaxation when the cardboard tube is removed. The gradient levels off during long tapping procedures. Fig. 4.7a shows the same analysis for exemplary samples prepared by the other protocols. For the strongly shaked $\mathrm{RAMP}_{\Gamma=5}$, a relative decompaction in the bottom part of the cylinder occurs. This might be explained by the complete lift-off of the packing during a single tap, and the following instantaneous "crash" to the bottom, restricting further rearrangements. Moreover, a strong radial decrease when approaching the cylinder wall is observed, but not accounted for in the analysis region. The VIB protocol shows extraordinarily high packing fraction near the bottom, where dense 


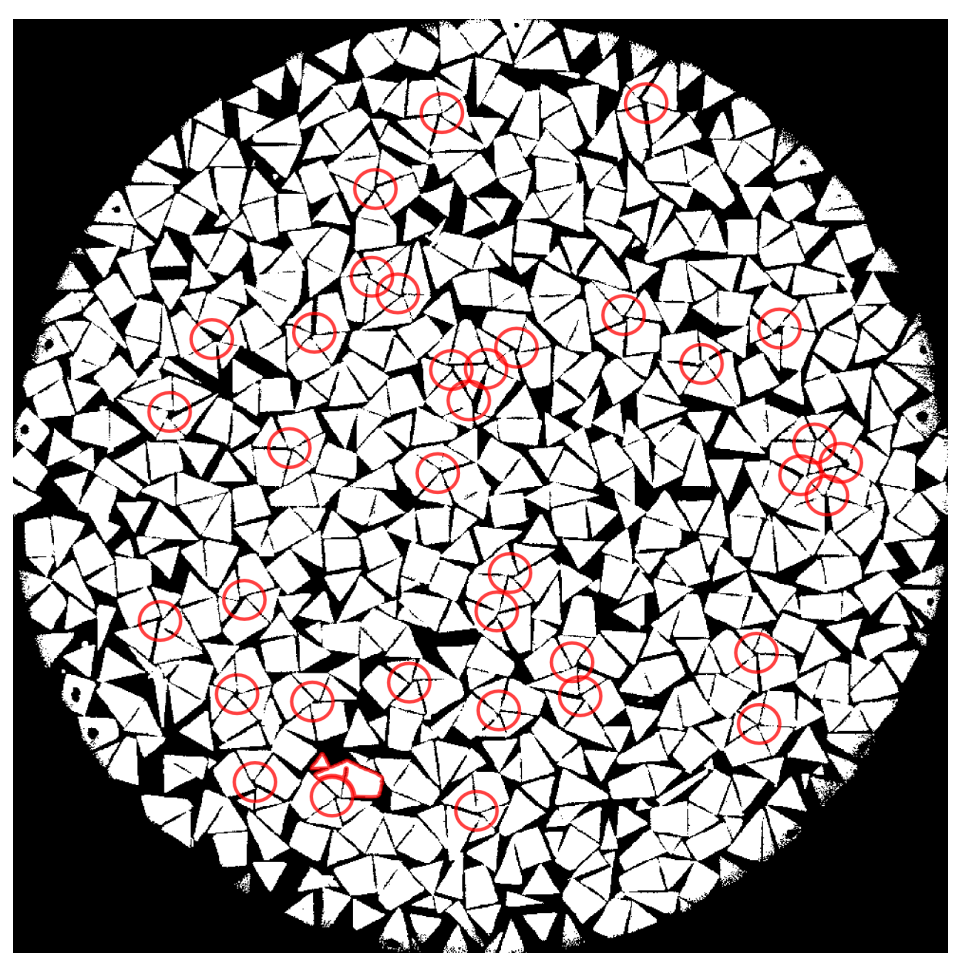

Figure 4.5: This VIB sample exhibits a higher structural order than other samples, which is apparent by visual inspection of the volume slices. Many pentamer structures are seen (marked as red circles), of which some are interconnected. The image is a Z-slice at $z=7 \mathrm{~mm}$ above the cylinder bottom, hence only pentamers oriented approximately in this plane are spotted.

clusters form (see also Fig. 4.5). Such locally high packing fractions are not found for any of the other protocols. 


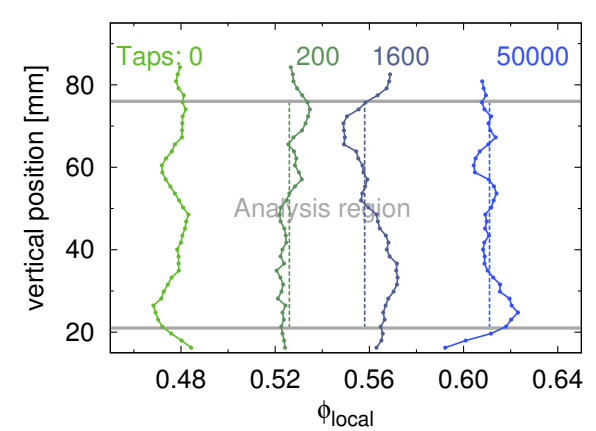

(a)

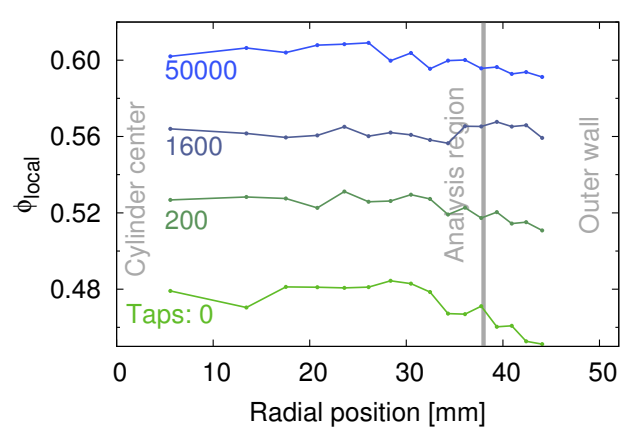

(b)

Figure 4.6: Spatial distribution of packing fraction for $\mathrm{TAP}_{\Gamma=2}$, with 0 to 50000 applied taps. Data points represent the center of bins where $\phi_{\text {local }}$ is averaged (sliding mean); each bin contains a few hundred particles. (a) Vertical density profile in analysis region (ROI), dotted lines represent the average $\Phi$. (b) Corresponding radial distribution of packing fraction in the cylinder with radius of $52 \mathrm{~mm}$, the radius of the analysis region analysis is indicated as a grey line.

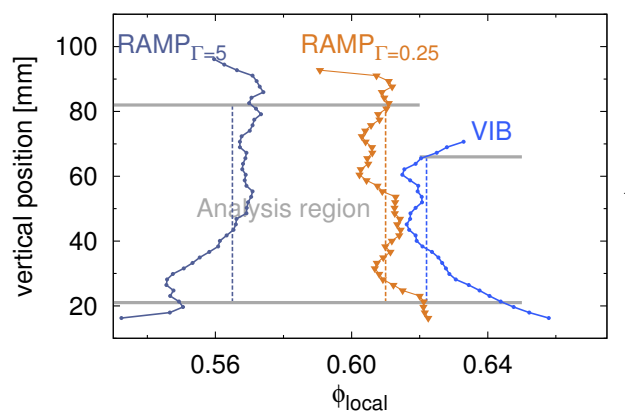

(a)

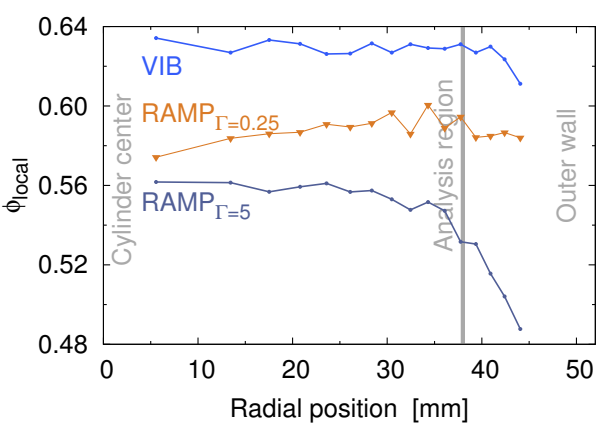

(b)

Figure 4.7: Spatial distribution of packing fraction for other preparation protocols. (a) The strongly shaken $\mathrm{RAMP}_{\Gamma=5}$ protocol shows a significant gradient from bottom(loose) to top(dense). RAMP $\Gamma=0.25$ is clearly more homogeneous. The VIB sample exhibits a extraordinarily high density near the bottom, which decays towards the top surface. This particular sample contains less particles than the others, so that the ROI is smaller. (b) The radial analysis confirms that $\operatorname{RAMP}_{\Gamma=5}$ has the most inhomogenous distribution of $\phi_{\text {local }}$, but the restriction to the inner analysis region with $38 \mathrm{~mm}$ radius discards the gradient near the outer wall. 


\subsection{Mechanical stability}

\subsubsection{Contact numbers}

The global contact number $Z$ is determined from the model-based CNS method as described in Sec. 3.2. This approach accounts specifically for the finite resolution of tomography and detection, but yields only a globally averaged contact number. Fig. 4.8 shows the results of different preparation protocols and packing fractions. Contact numbers of protocol TAP start at the loose, initial packing with $Z$ between 6.5 and 7 , then increase monotonically with $\Phi$ and level off at $\Phi \approx 0.6$. The two densest packings, $\operatorname{RAMP}_{\Gamma=0.25}$ and VIB do not show a further increase of $Z$. However, the packings prepared by $\operatorname{RAMP}_{\Gamma=5}$ deviate distinctly: For comparable $\Phi$, contact numbers are approx. 1.0 lower, underlining the influence of the preparation protocol.

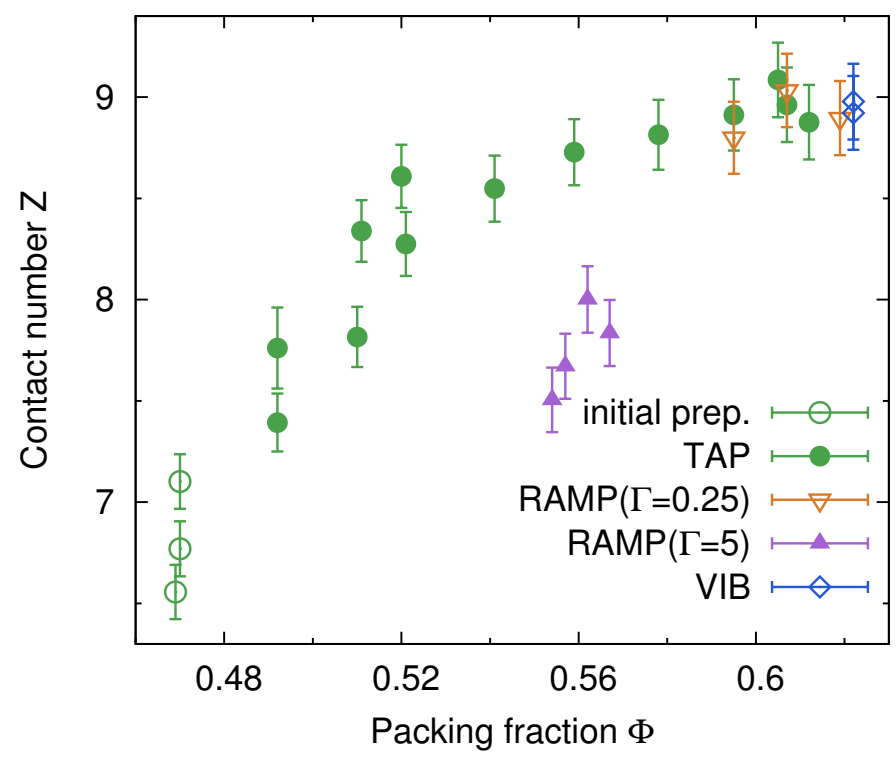

Figure 4.8: Global contact number $Z$ vs. packing fraction $\Phi$ for different preparation protocols. Contact numbers of $\mathrm{TAP}_{\Gamma=2}$ samples vary smoothly from the initial packing to the densest packing ( $10^{5}$ taps).

The value of $Z$ and $\Phi$ at high packing fractions in Fig. 4.8 compare surprisingly well to the Jamming density $\Phi_{J}=0.61$ and contact number $Z_{J}=8.6$, which were found in simulations of frictionless tetrahedra (Fig. 1.2, $[80])$. 


\subsubsection{Protocol dependency of contact numbers}

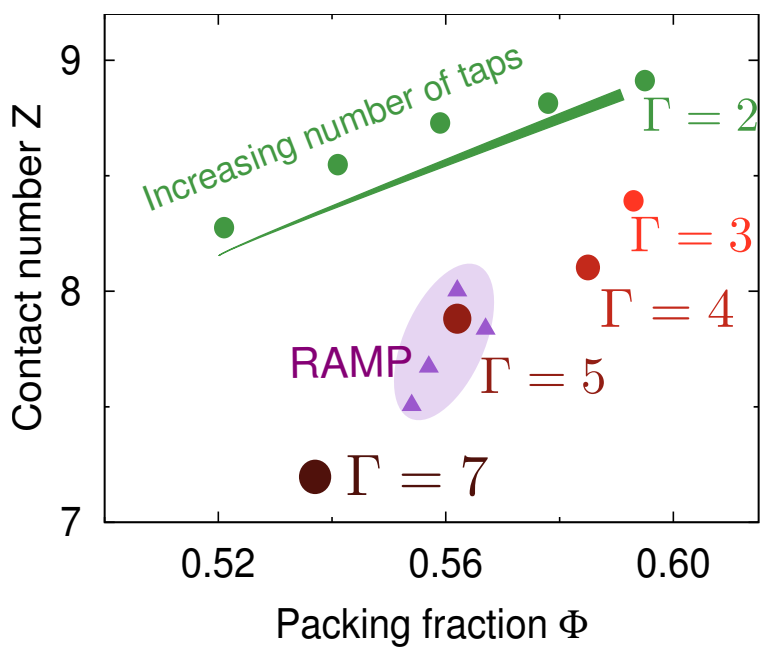

Figure 4.9: Contact number $Z$ of packings after 10000 applied taps with different tap intensity $\Gamma$ (circles with $\Gamma$ increasing from bright red to dark). TAP samples at $\Gamma=2$ (green dots) and similar packing fraction and $\mathrm{RAMP}_{\Gamma=5}$ samples (violet triangles) are shown for comparison. Colored regions are guides to the eye, referring to the $\operatorname{TAP}(\Gamma=2)$ and RAMP datapoints. Note that the range of $\Phi$ covered here is smaller than in Fig. 4.8.

For a systematic study of the deviations and a potential history dependency of protocol $\mathrm{RAMP}_{5}$, samples were tapped for $10^{4}$ times with varying $\Gamma=3 \ldots 7$, and then tomographically analysed. The contact number of these samples (last datapoints in Fig. 4.3) decreases strongly with $\Gamma$ and develops an additional branch in the $Z(\Phi)$-diagram. Apart from possible static charging effects (Sec. 4.1.4), the higher intensity might indeed create different structures, which have the same $\Phi$, but different $Z$. The left-skewed distribution of $\phi_{\text {local }}$ (4.23) shows that strong shaking at $\Gamma=7$ causes a broader distribution of local packing fractions, compared to a $\mathrm{TAP}_{\Gamma=2}$ sample, even if the global $\Phi$ is the same.

\subsubsection{Global contact types and constraints}

The contact number $Z$ of tetrahedra packings is not sufficient to characterize its distance to isostaticity. Contrary to sphere packings, the number of imposed mechanical constraints depends on the specific contact geometry, as outlined in Sec. 3.3. Fig. 4.10 shows that the number of all four types of contacts increases with $\Phi$. More specifically, the growth of the EF contacts contributes about half of the total increase in $Z$. Together, the number of constraints per tetrahedra is between 12 and 18 as Fig. 4.11 shows, and therefore much higher than the isostatic limit of 6 . While this result 
does not contradict the isostatic packings found in simulations of frictionless tetrahedra $[79,80,37]$, there is a clear disagreement with the experiments using frictional dice reported in [34]. There, however, the authors use the contact-specific constraint numbers pertinent to frictionless particles. If the frictional multiplication factors are assumed, the resulting constraint number in Ref. [34] is also hyperstatic. On the other hand, if frictionless factors are used for the analysis of our data, the loosest packings become hypostatic which does not agree with the observed mechanical stability.

Frictional tetrahedra packings are therefore hyperstatic, as measured by the generalized constraint number $C$. For a given packing fraction, a range of contact and constraint numbers is compatible with mechanical stability, and depends on the preparation protocol. These new observations challenge contemporary theoretical approaches to static granular media - e.g. the proposed inverse relationship between mean free volume and contact number, which is independent of preparation details [82]. Similarly, the jamming paradigm $[44,90]$ is based on a single power law relationship between $Z$ and $\Phi$ and does not account for protocol dependent variations or friction [77].

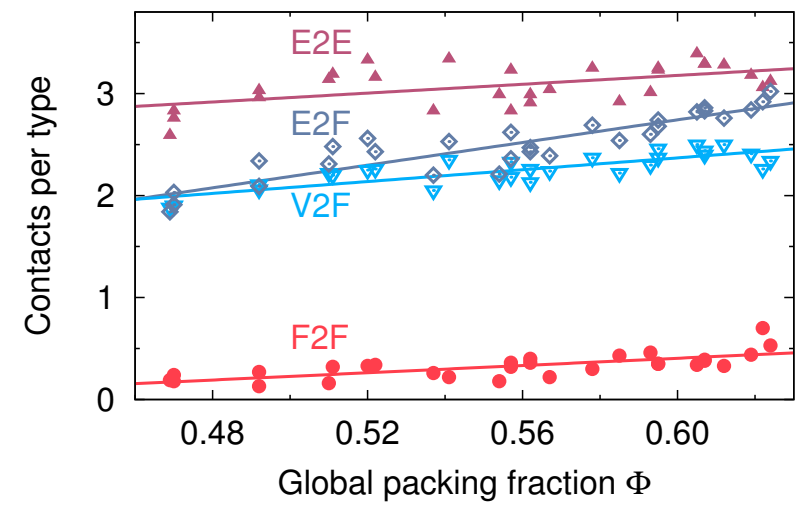

Figure 4.10: Number of contact types (global average) of all samples with different $\Phi$, namely face-face contacts $Z_{F F}$, edge-face contacts $Z_{E F}$, vertex-face contacts $Z_{V F}$ and edge-edge contacts $Z_{E E}$.

\section{Distribution of contact types}

Fig. 4.12 show the distribution of face-face contacts and point contacts (sum of vertex-to-face and edge-to-edge contacts) for selected samples, with consistent symbol and color encoding. While the loose (initial) sample has a low probability of only $20 \%$ to find a face-face contact, the densification process by the $\operatorname{TAP}(\Gamma=2)$ or the VIB protocol increases the number of particles with at least one face-face contact (=dimers or larger) strongly (Fig. 4.12a). It is noteworthy that the distribution of $Z_{F F}$ for the strongly shaken sample $\mathrm{TAP}_{\Gamma=7}$ closely resembles that of a initial (loose) packing; it even has the 


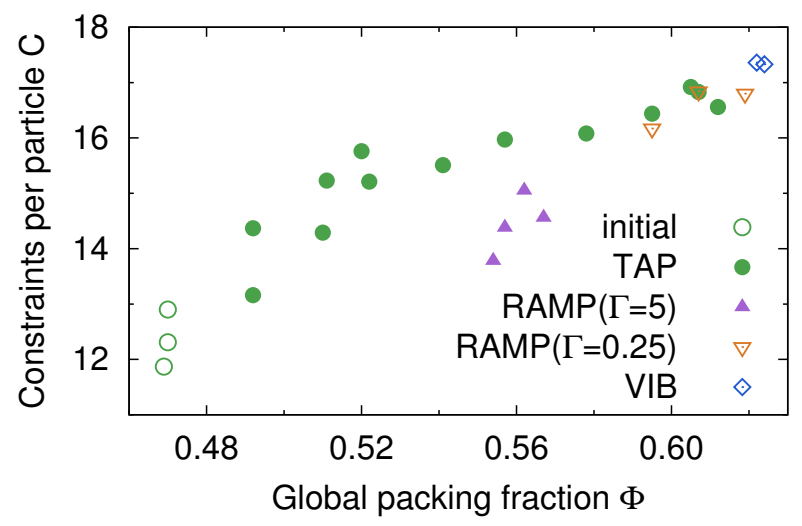

Figure 4.11: Generalized constraint numbers $C$ per particle, obtained by weighting the contact types Fig. 4.10 with the according constraint factors. The preparationdependent variation as in $Z(\Phi)$ persists (Fig. 4.8), but whereas the contact number $Z$ levels off at $\Phi \approx 0.60$, the constraint number $C$ increases further.

same probability of $p=0.06$, despite a much higher global density $(\Phi=0.53$ vs. $\Phi=0.47)$.

If $Z_{F F}$ is interpreted as a random variable, the probability of a tetrahedron having $k$ face-face contacts can be modeled by a binomial distribution $B(n, k, p)$, where the number of trials is $n=\max (k)=4$ and $p$ is a sampledependent probability. The lines in Fig. 4.12a, where $p$ is determined as fit parameter to the data points, show that the binomial distribution models the number of face-face contacts very well.

This leads to the conclusion that the formation of face-face contacts follows a independent random process. In other words, the probability for additional FF contacts does not change, once a dimer or trimer has been formed; the process depends only on the probability of a single face-face contact to occur. When considering the dense analytically or numerically constructed packings (??), the random model obviously breaks down, because these packings have a deliberately fixed number of face-face contacts. Indeed, the onset of deviations from the random model could serve as the key criterion for characterizing the transition between disorder and order in tetrahedra packings.

The distribution of point contacts, as the sum of $Z_{V F}$ (vertex-face) and $Z_{E E}$ (edge-edge), is shown in Fig. 4.12b. These contacts are not modeled by a Binomial as above, because the maximum number of point contacts (resp. bonds in the lattice) and geometric relations or restrictions between $Z_{V F}$ and $Z_{E E}$ are unclear.

Percolation theory provides further insight about the structure of faceface contacting clusters: the face-face geometry of tetrahedra is appropriately modeled by a diamond lattice in $3 \mathrm{D}$, where every site can have between 


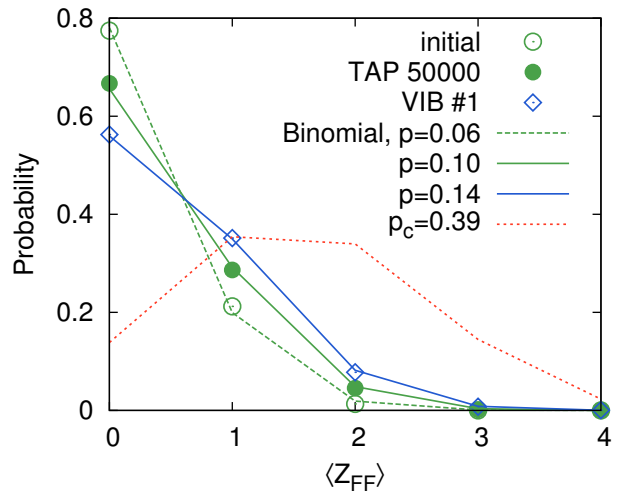

(a)

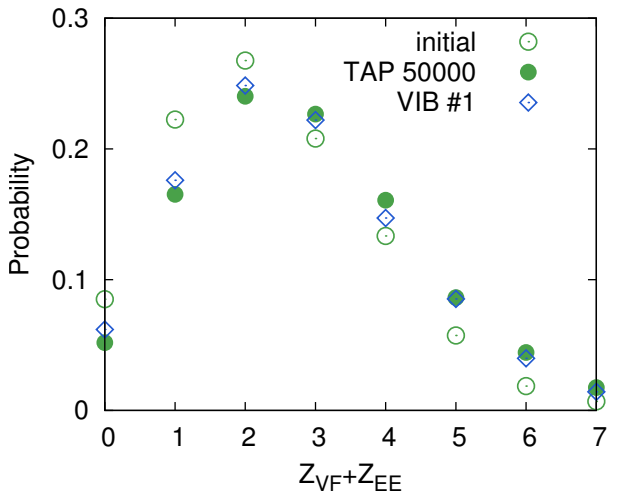

(b)

Figure 4.12: (a) Observed probability face-face contact number $Z_{F F}$ in three packing samples with different preparation protocol (data points). The lines are binomial distributions, with the face-contact probability $p$ as a fit parameter (Details see text). All samples are far from percolation, because the distribution for $p_{c}=0.39$ deviates distinctly (dotted red line). (b) Distribution of "lower order" point contacts (sum of vertex-to-face and edge-to-edge contacts): Minor deviations between initial (loose) packing and sample $\mathrm{TAP}(\Gamma=7)$ are found, the denser samples have more point contacts.

0 and 4 bonds - respectively F2F contacts - to its neighbors. Each bond is assigned the probability $p$ to be present, respectively $1-p$ to be missing. If $p$ is larger than the bond-percolation threshold $p_{c}$, a connected cluster emerges $^{1}$, which spans the full system size. For the diamond lattice, the threshold was numerically determined to $p_{c}=0.390$ [92]. Therefore, percolation of face-face contacts in tetrahedron packings is expected if the average face-face contact number $\left\langle Z_{F F}\right\rangle$ exceeds $4 \cdot p_{c}=1.56$.

\footnotetext{
${ }^{1} p_{c}$ is defined as the value, where percolation occurs in more than $50 \%$ of all system realizations.
} 


\subsection{Characterization of geometric order}

\subsubsection{Translational order}

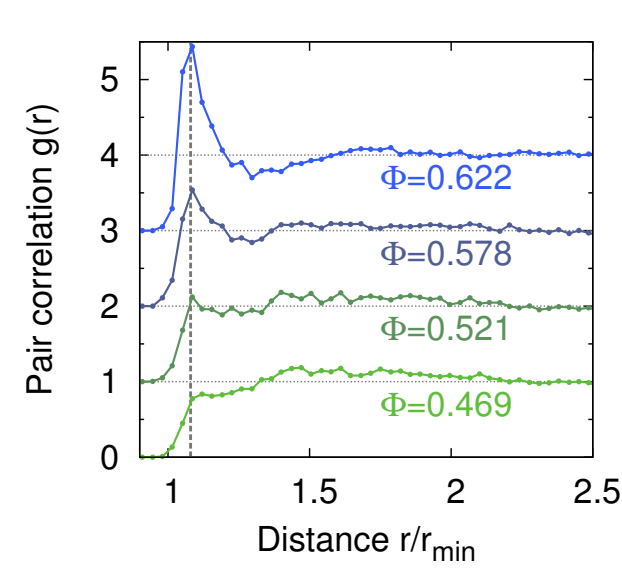

(a)

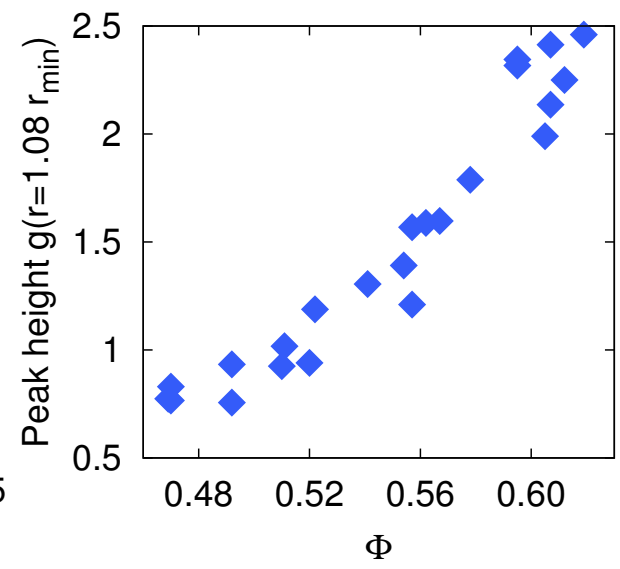

(b)

Figure 4.13: (a) Pair correlation function $g(r)$ for 4 different packing fractions. Offsets are added for clarity (lower three samples: initial and TAP protocol, highest: VIB protocol). (b) Peak height as indicated by dotted line in (a) at $r=1.08 r_{\text {min }}$ (all samples)

A measure commonly used for characterizing order in disordered matter, like granulates, glasses or liquids is the pair correlation function $g(r)$. It counts the number of particles in the radial distance shell $[r, r+\delta r]$, normalized by the binning volume and the average density. This implies that $g(r)$ equals 1 , if no significant density variations are present. If $g(r)$ exhibits peaks exceeding 1, particles arrange denser than on average, which points to a close arrangement of neighbors at the distance $r$. Fig. 4.13a shows $g(r)$ for samples with different density $\Phi$. The first peak is located at $r=0.44 a=1.08 r_{\text {min }}$ (dashed line) and grows monotonically towards denser packings. The growing peak height can be explained by an increasing alignment of face-face or acute edge-face contacts. The peak position is slightly offset from the minimal possible distance of twice the inradius $\left(2 R_{\text {in }}=r_{\min } \approx 0.408 a\right)$, because the probability to observe acute face-to-edge or laterally shifted face-face configurations is higher than for perfectly aligned faces. $g(r)$ decays quickly after the first peak, showing no long-range translational ordering. This is in contrast to the $g(r)$ obtained from Monte Carlo (MC) simulations of tetrahedra $[26,37]$ which revealed long-range structure at densities as low as $\Phi=0.46$. The difference is due to the preparation technique: the MC simulations do not account for gravity or friction, but compress a gas or liquid phase of tetrahedra particles in an isotropic way. In contrast, the packings in the experimental setup jam due to gravity already at $\Phi \approx 0.47$, after which 
geometric frustration and pressure in the pile restrict further alignment.

The left shoulder in $g(r)$ has a finite slope, because tetrahedra can be in contact for a range of distances, from twice the inradius to twice the circumradius, in contrast to mono-sized spheres. This has implications regarding the Jamming transition: Simulations of soft frictionless spheres have revealed that the first neighbor peak in $g(r)$ scales as $g(r) \sim \frac{1}{\sqrt{r-1}}$ (for spheres with radius 1) [90, 78]. Thus, when approaching the Jamming point from below, that is, $r \rightarrow 1$, a divergence of the $g(r)$ peak is found. ${ }^{2}$ However, the broadness of the $g(r)$-peaks in Fig. 4.13a - a consequence of the variation in contact distances - suggests that packings of soft tetrahedra under compression will differ from this scaling behaviour and not show this singularity near Jamming.

\subsubsection{Orientational order}

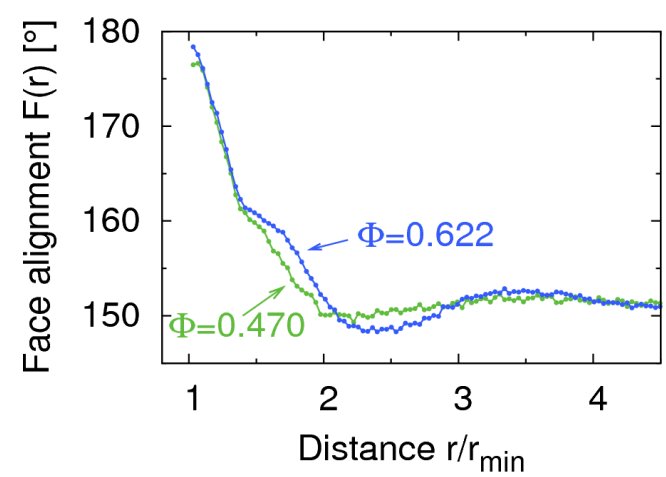

Figure 4.14: Face correlation $F(r)$, normalized to the minimal possible distance of twice the inradius $\left(r_{\min }=2 R_{i n}\right)$. Loose samples (initial preparation) shows a quick decay of orientational ordering after the first neighbor. Correlations in the dense sample (protocol VIB) level off after the next-nearest neighbor at $r / r_{\min } \approx 3$.

An estimator for orientational order is the angular correlation function $F(r)$, which measures the average relative orientation of face normals in the distance $r$.[34]. $F(r)$ is computed by averaging $F_{q l}$ for all pairs of tetrahedra $q$ and $l$ whose centroids $\overrightarrow{c_{q}}$ and $\overrightarrow{c_{l}}$ are a distance $r$ apart. $F_{q l}$ is then defined as the minimum of the pairwise dot product of the 4 face normals $\vec{n}_{q, 1 \ldots 4}$ and $\vec{n}_{l, q \ldots 4}$ :

$$
\begin{aligned}
F(r) & =\left\langle F_{q l} \delta\left(\left|\overrightarrow{c_{q}}-\overrightarrow{c_{l}}\right|-r\right)\right\rangle_{q l} \quad \text { with } \\
F_{q l} & =\min \left(\vec{n}_{q, 1 \ldots 4} \cdot \vec{n}_{l, 1 \ldots 4}\right) .
\end{aligned}
$$

\footnotetext{
${ }^{2}$ One should note that these simulations are implemented by "inflating" all spheres simultaneously, which is not reproducible experimentally for hard tetrahedra particles.
} 
Thus, the ideal face-face alignment corresponds to $F(r)=180^{\circ}$, whereas a completely random configuration has $F(r)=150^{\circ}$. Fig. 4.14 compares the face alignment of a loose and a dense packing. Orientational order extends slightly further than translational order, which is in good accordance with previous experiments [34] and numerical results [79]. The development of a shoulder for the densest packing, starting at $r \approx 1.6 r_{\text {min }}$ indicates the emergence of trimers (3 tetrahedra aligned face-face), which have a (ideal) centroid distance of $r=1.63 r_{\text {min }}$.

One should note that $F(r)$ only accounts for the pair-wise alignment of face normals, but not for in-plane rotations of the faces against each other. This is considered in the correlation function $C(r)$, which is averaged radially over particles pairs $q, l$ as above:

$$
\left.C(r)=\left\langle C_{q l}\right\rangle=\left\langle\frac{1}{4} \sum_{i=1}^{4} \vec{n}_{q, i} \cdot \vec{n}_{l, i}\right)\right\rangle .
$$

Here, the normal vectors $\vec{n}_{i}^{q}$ and $\vec{n}_{i}^{l}$ are ordered in the sense that always the corresponding pair of vectors are computed; these are the vectors which maximize the pair-wise scalar product. Thus, $C(r)$ tells us if particles in a distance $r$ are superposable i.e. are identically oriented. For tetrahedra packings, $C(r)$ matches closely the signature and correlation length as in $F(r)$ (see e.g. $[34,37,76]$ ). In conclusion, neither of these radially averaged correlation functions is sensitive enough to establish a clear link between local structures and global packing fractions.

\subsubsection{Densest arrangements}

The key questions addressed in this section are: Which dense structures are attainable experimentally in disordered tetrahedra packings? How do they possibly relate to structures which are known from analytical or numerical work? In the recent sections, we have already seen indications for an increasing formation of dimers, trimers and pentamers, which are all face-face connected clusters.

\section{Face-face contacting clusters}

A face-face configuration is preferred for two reasons: Firstly, packing fraction is locally maximized when the centroids are closest to each other, which is the case for an aligned dimer. While these are probably the globally densest configurations, it is very unlikely to observe the dimer crystal in experiments, because it occurs only at extremely high pressures in MC simulations. Secondly, face-face contacts maximize mechanical stability by fixing more constraints at once than any other contact. Fig. 4.15 shows the distribution of face-face cluster sizes for a loose (initial preparation) and a dense sample $\left(10^{5}\right.$ taps at $\left.\Gamma=2\right)$. The distribution is exponential in good 
approximation, and in the denser sample, more larger clusters are found. Exemplary clusters containing 5 or 6 tetrahedra are illustrated in Fig. 4.16.

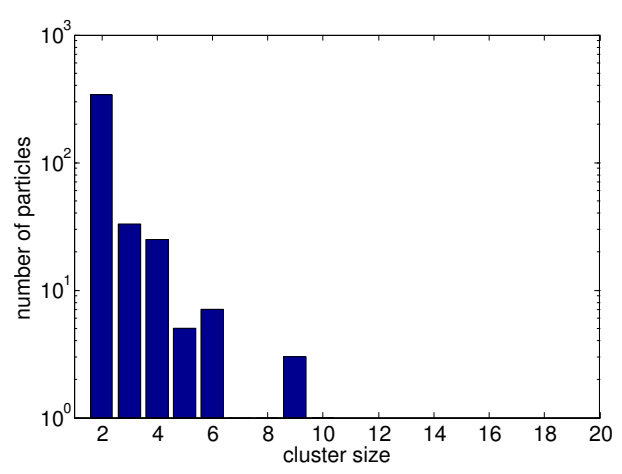

(a)

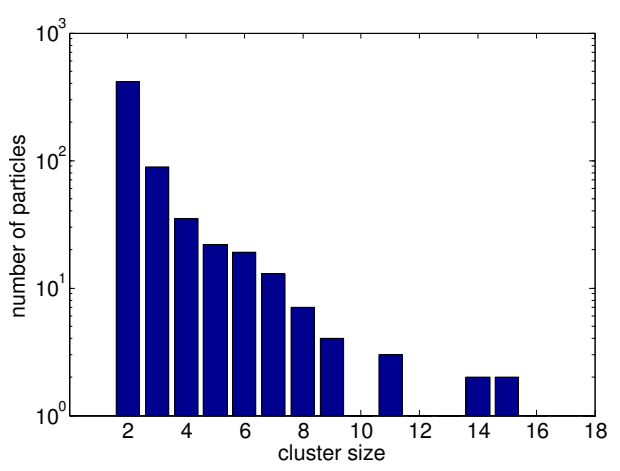

(b)

Figure 4.15: Distribution of face-face-contacting cluster sizes (logarithmic scale) for (a) Loose packing at $\phi=0.470$ (b) Dense packing (TAP) at $\phi=0.612$, showing the increasing number of clusters larger than 3 particles (trimers). The total number of particles in the ROI is comparable $(\approx 3500-4000)$.

These pentamer clusters could be interpreted as "local crystallites" in disordered tetrahedra packings, and dense packings strongly resemble the disordered wagon-wheel-packing [86]. A general criterion for detecting pentamers would be desirable, but this requires a certain acceptance threshold. In MC simulations from Haji-Akbari et al. , tetrahedra under compression increasingly arrange into pentamers[26], but the absolute number found in these clusters varies strongly, depending on the chosen threshold. Therefore, only the relative occurence of pentamers for a fixed (arbitrary) threshold can be analysed.

Furthermore, the visual inspection of connected clusters revals that cluster geometries range from compact pentamers to extended face-face-connected chain, as is illustrated in Fig. 4.17.

In one of the VIB samples, local icosahedral order is observed, as depicted in Fig. 4.18. It is practically difficult to grow dense structures further than the first layers on the bottom, because convection sets in easily and destroys the order again. A fixed bottom layer in the shape of a quasicrystal unit cell [26] might serve as a seed for growing a quasicrystal experimentally ${ }^{3}$.

\section{Smallest Voronoi volumes}

Another possibiliy to find the dense building blocks of disordered tetrahedra packings is the investigation of cells with the smallest Voronoi volume $V$. From a geometric argument, the densest local structure is achieved if each face of a tetrahedron is covered by 4 perfectly aligned facets of the neighbors.

\footnotetext{
${ }^{3}$ Discussion with P. Damasceno
} 


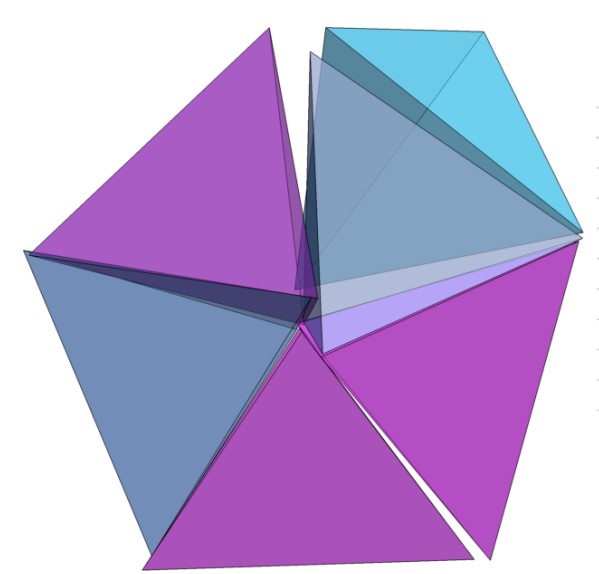

(a)

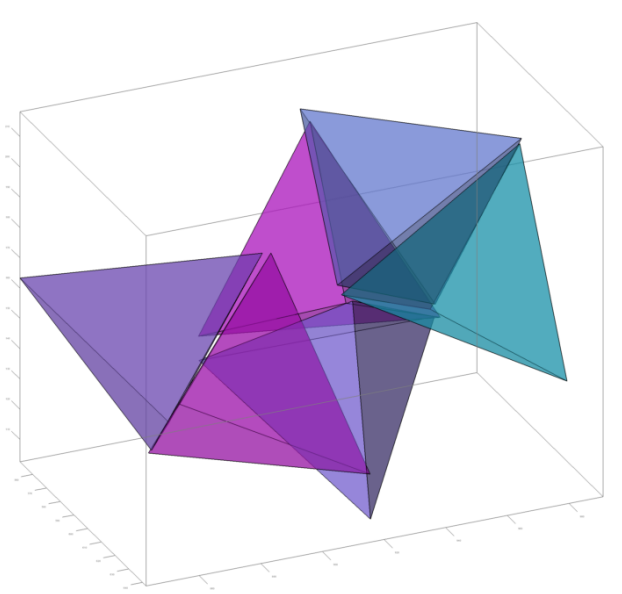

(b)

Figure 4.16: Observed clusters containing both 6 FF-jointed tetrahedra in sample VIB \#1 $(\phi=.622)$. (a) Pentamer with an additional FF contact (b) Extended FF chain consisting of two connected, imperfect pentamers. Coloring is arbitrary for better visibility.

This is the case e.g. for the one tetrahedron being enclosed in the nonamer structure and shared between two pentamers (see Sec.1.3 for illustrations).

A search for tetrahedra with the highest $\phi_{\text {local }}$ among all samples revealed that a nonamer structure exists in one of the globally densest packings $\left(\mathrm{RAMP}_{\Gamma=0.25}\right)$, see Fig. 4.19. The coloring is according to local packing fraction, mapped relative to the specific sample distribution. The central tetrahedron with $\phi_{\text {local }}=0.82$ is occluded, because it is covered from four sides by the two intertwined pentamers. 


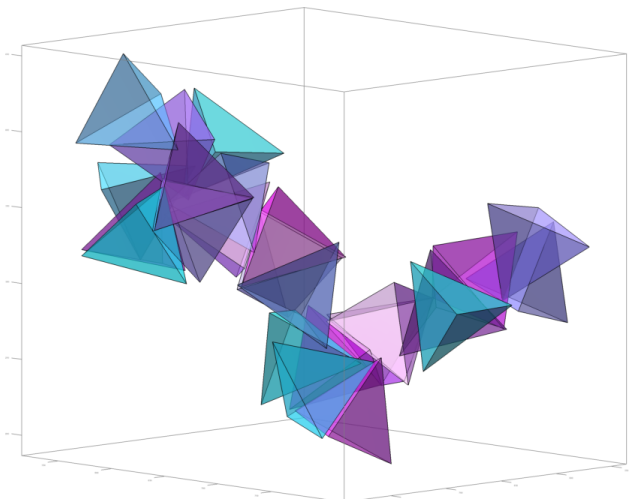

Figure 4.17: A large face-face-cluster spanning 24 tetrahedra is detected in sample TAP, $10^{5}$ taps applied, $\Phi=0.610$

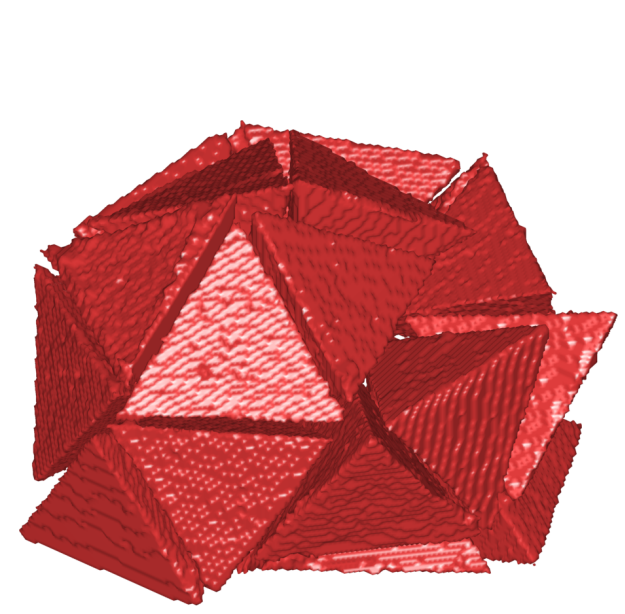

(a)

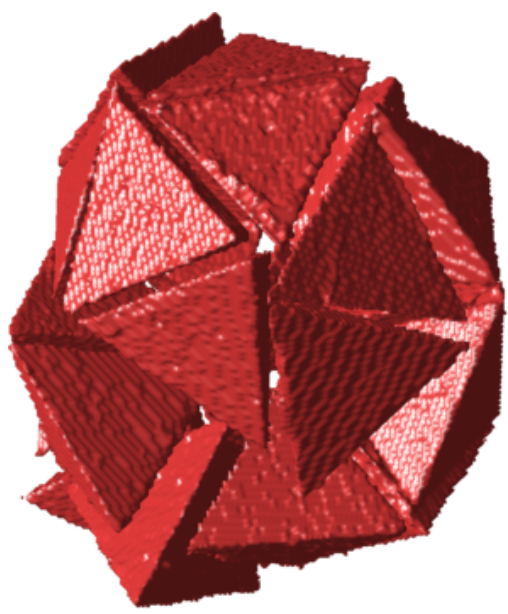

(b)

Figure 4.18: Distorted icosahedral arrangement of tetrahedra as observed in sample VIB, $1 \mathrm{~cm}$ above the cylinder bottom. (a) Side view (b) Top view

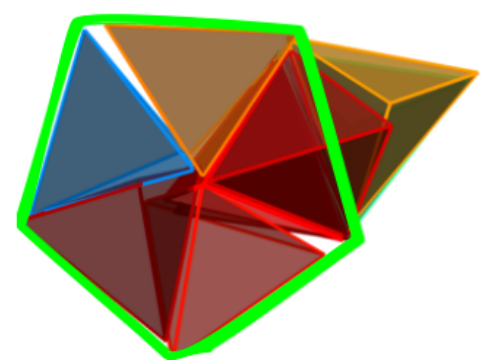

(a)

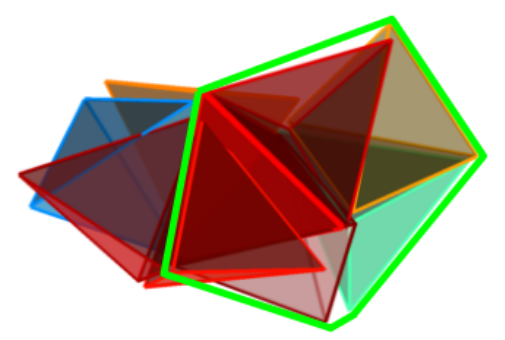

(b)

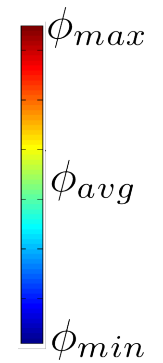

Figure 4.19: Nonamer structure, shown from two perspectives: Central tetrahedron $\left(\phi_{\text {local }}=0.82\right)$ is shared between two pentamers. Coloring relative to distribution of local packing fraction $\left[\phi_{\min } \ldots \phi_{\max }\right]$. (a) First pentamer (marked green) (b) Second pentamer, from a different perspective, approx. $90^{\circ}$ turned (marked green) 


\section{Cluster participation ratio}

Recently, Li et al. proposed the participation ratio $\rho$ as an order parameter for tetrahedra packings [76]. $\rho$ is defined as the fraction of tetrahedra with at least one "face-face joint" (face-face contact), and is therefore a lower bound for the average number of face-face contacts. They showed that the packing density $\Phi$ is proportional to $\rho$ for a variety of different numerically prepared packings (among others, the disordered wagon-wheels, the quasicrystal and the dimer packings). If extrapolated to $\rho=0$, the lower bound of $\Phi=0.625$ is proposed as the densest disordered packing fraction, in analogy to the random close packing limit of spheres $(\mathrm{RCP})^{4}$. A linear extrapolation to $\rho=1$ coincides with the maximum packing density of $\Phi=0.856$ for the dimer packing[12].

The participation ratio in our experimental tetrahedra packings varies between 0.14 and 0.6 , but no clear trend with packing fraction is observed. However, the difference between the global face-face contact number $Z_{F F}$ and the participation ratio (see Fig. 4.20) exhibits a jump at $\Phi \approx .62$ for the VIB samples. This supports the idea that under compaction, samples undergo a transition from the formation of separated dimers, where $Z_{F F} \approx \rho$, to the growth of larger face-face-clusters. This is corroborated by the cluster size distributions in Fig. 4.15. The process of face-face contact formation reminds of a classical nucleation and growth process. In this context, modeling approaches could possibly compute nucleation barrier energies for different geometric configurations.

The strong increase of $Z_{F F}-\rho$ at $\Phi \approx .62$ supports the hypothesis by $\mathrm{Li}$ et al., that the amount of FF clusters is a valid order parameter despite the large differences between experiment and numerical simulation, regarding particle shape, preparation or friction. In principle, the face-face contact number is more sensitive to structural changes, because it accounts for multiple face-joints per tetrahedron, and should therefore be favoured over the suggested participation ratio [76].

\subsection{Local properties}

The variation in contact and constraint number at the same global packing fraction might be explained by different internal packing structures. Therefore, a analysis of local structures is needed, covering local packing fractions, local contact geometries, and analysis of contacting clusters (see e.g. [76]).

\footnotetext{
${ }^{4}$ In contrast, Torquato[86], who proposed the "maximum random jammed" (MRJ) state for the densest disordered sphere packing, claims that disordered tetrahedra packings have $\Phi_{M R J}=0.763$. A different conception of "order" or "disorder" is the most likely explanation for these vastly differing values, which emphasizes again the difficulty in finding good order metrics.
} 


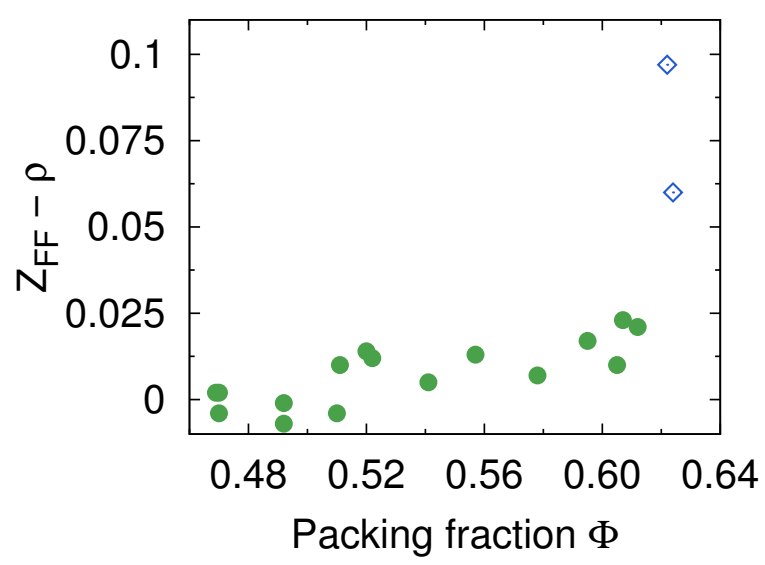

Figure 4.20: Difference between average face-face contact number $Z_{F F}$ and participation ratio $\rho$. For the TAP samples, a slow increase with packing fraction is observed, but the VIB samples above $\Phi \approx .62$, deviate strongly.

\subsubsection{Local packing fraction from Voronoi volumes}

The local packing fractions $\phi_{\text {local }}$ are accessible via the associated Voronoi volume $V$ of each particle (Sec. 1.2) and the known (fixed) tetrahedra volume of $V_{\text {tet }}=40.42 \mathrm{~mm}^{3}$ as $\phi_{\text {local }}=V_{\text {tet }} / V$. The globally averaged $\Phi$ is computed as the ratio between the volume occupied by all particles and the total Voronoi volume ${ }^{5}: \Phi=\sum_{i} V_{p}^{i} / \sum_{i} V^{i}$.

Fig. 4.21 shows the distribution of the local packing fractions $\phi_{\text {local }}$ of a loose and a dense sample, fitted by a Gaussian distribution. Whereas the Gaussian matches the experimental distribution on first sight, a closer look reveals that $V$ is not distributed symmetrically, but skewed to the right. The skewness ${ }^{6}$ increases systematically from 0.5 to 2.2 between $\phi=0.47$ and $\phi=0.62$. This means that compaction causes the number of locally dense structures to increase stronger than the number of loose structures is reduced - otherwise, we would observe a shifted Gaussian. A consequence of the increasing skewness is that a rescaling of the distributions to a Gaussian by mean and variance is likely to fail.

A different distribution was suggested and theoretically motivated by Aste et al. [2]: They showed that the distribution of free (or excess) volume $V-V_{\min }$ in sphere packings, when normalized appropriately, follows a Gamma-distribution. The probability density function has two parameters, $k$ ("shape") and $\Theta$ (scale) and follows the functional form (for $k \in \mathbb{Z}$ ):

$$
f(x \mid k, \Theta)=\frac{x^{k-1} e^{-x / \Theta}}{\Theta^{k}(k-1) !}
$$

\footnotetext{
${ }^{5} \mathrm{~A}$ common pitfall is the assumption that $\Phi=\left\langle\phi_{\text {local }}\right\rangle$

${ }^{6}$ third moment of the distribution
} 


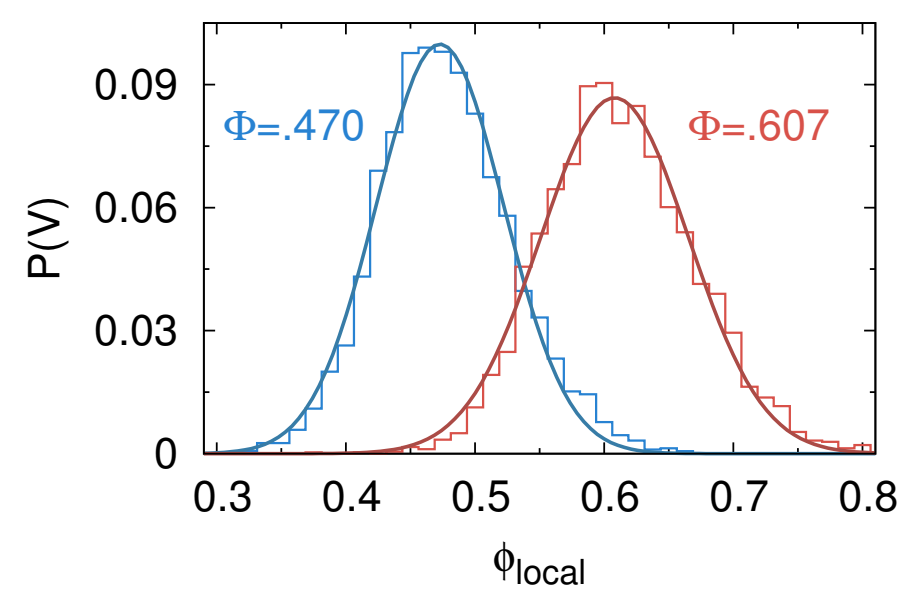

Figure 4.21: Distribution of local packing fraction for loose (initial) and dense sample prepared by the TAP protocol (blue and red steps), the legend denotes the global $\Phi$. Gaussian fits (lines), with parameters $\mu=0.473, \sigma=0.05$ (loose) and $\mu=0.608, \sigma=0.06$ (dense).

For the special cases of $k=1$, the exponential distribution is obtained, and for $k \rightarrow \infty$, the Gamma distribution converges to a Gaussian, changing its shape smoothly between these two limiting cases. The choice of this distribution is motivated by the assumption that each Voronoi cell can be decomposed into a certain number of sub-cells, which follow an exponential size distribution. If that holds, the summed volume of $k$ randomly picked sub-cells should follow a universal Gamma-distribution, with the shape parameter $k$ and the scale parameter $\theta=\left(V-V_{\min }\right) / k$.

Regarding the tetrahedra volume (resp. packing fraction) distributions in Fig. 4.21, the asymmetric Gamma distribution is expected to capture the skewness feature better. For a accurate comparison, the aggregated Voronoi volume distribution of all samples is normalized and fitted separately by a Normal (Fig. 4.22a) and a Gamma distribution (Fig. 4.22b). Judging from two statistical test (the Kolmogorov-Smirnov-test and the squared sum of residuals), the Normal distribution remains slightly superior, but significant deviations remain for both models. More specifically, the symmetric Gaussian deviates equally on both tails of the data distribution. In contrast, the Gamma distribution is off-centered and overestimates the number of larger volumes (resp. low packing fractions). Furthermore, a universal $k$-value cannot be established, because it decreases systematically from $k=26$ for the loosest packing to $k=18$ for the densest packing.

How can we interpret the mismatch with the models? Firstly, the original assumptions might be violated, e.g. the choice of the Gamma function. This choice was motivated by the exponential size distribution of the sub-volumes - which in turn, is a consequence of the Poisson statistics of randomly distributed point clouds. Since the Voronoi volumes from Aste 
et al. collapse to a Gamma distribution, the generalization from points to mono-sized spheres in mechanical contact appears still valid. In contrast to sphere packings, tetrahedra do not have a "typical" centroid distance, but this varies by a factor of 3 between twice the inradius $2 R_{\text {in }}$ and twice the circumradius $2 R_{\text {out }}=2 \cdot 3 R_{\text {in }}$. Thus, the model is pushed even further: From points (no restriction on the pair-wise distance) to spheres (fixed distance at contact) and to tetrahedra (range of distances at contact)

Secondly, the random drawing of Voronoi volumes assumes statistically independent sets; if spatial correlations are present, this assumption is violated. For example, correlations of the Voronoi volumes in binary disc packings extend as far as 5 particle diameters (Zhao and Schröter[97]). Even if the structural order of the experimental tetrahedra packings is much shorterranged than for sphere packings, spatial correlations are supposedly still relevant.

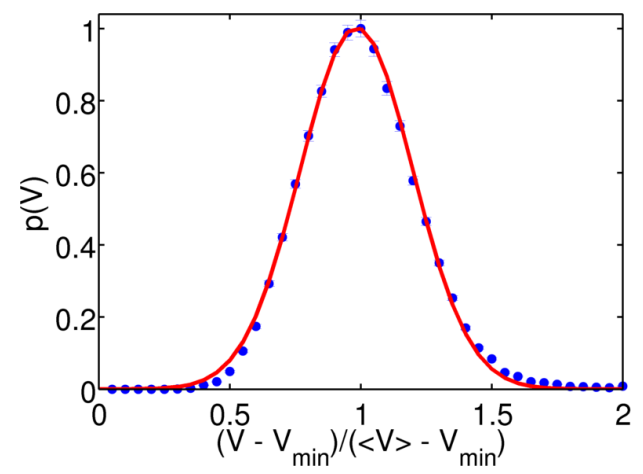

(a)

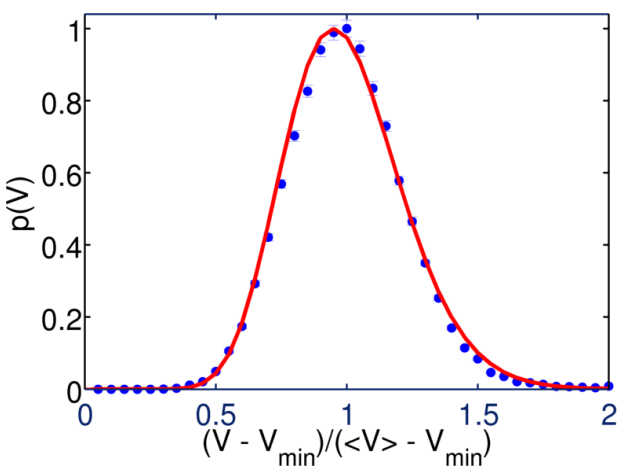

(b)

Figure 4.22: Aggregated Voronoi volume distributions of all packings, rescaled by minimum possible volume $V_{\text {min }}=V_{\text {Tet }}$ and average volume $\langle V\rangle$. (a) Fit of Gauss distribution with $\mu=0.98, \sigma=0.21$ (b) Fit of Gamma distribution with $k=20, \theta=0.05$.

\section{Influence $\Gamma$ on volume distribution}

The distribution of $\phi_{\text {local }}$ could shed light onto the protocol-dependent differences between the gently tapped sample $\mathrm{TAP}_{\Gamma=2}$ and the vigourously shaken samples $\operatorname{RAMP}_{\Gamma=5}$ or TAP $\mathrm{T}_{\Gamma=7}$. Fig. 4.23 illustrates a difference between the $\operatorname{TAP}_{\Gamma=7}$ sample and a $\operatorname{TAP}_{\Gamma=2}$ sample with comparable $\Phi$. Although the global packing fractions are very close $(\Phi=.537$ resp. $\Phi=.541)$, the distributions differ significantly: The $\mathrm{TAP}_{\Gamma=7}$ packing has more very loose regions down to $\phi_{\text {local }} \approx 0.37$ and the overall distribution is broader, as measured by the standard deviation of the fitted Gaussian. Is only the larger inhomogeneity of $\phi_{\text {local }}$ in the $\mathrm{TAP}_{\Gamma=7}$ sample responsible for this deviation? A contraindication to this explanation is however the robustness of the feature 
throughout the sample, e.g. for different radial or vertical analysis subsets.

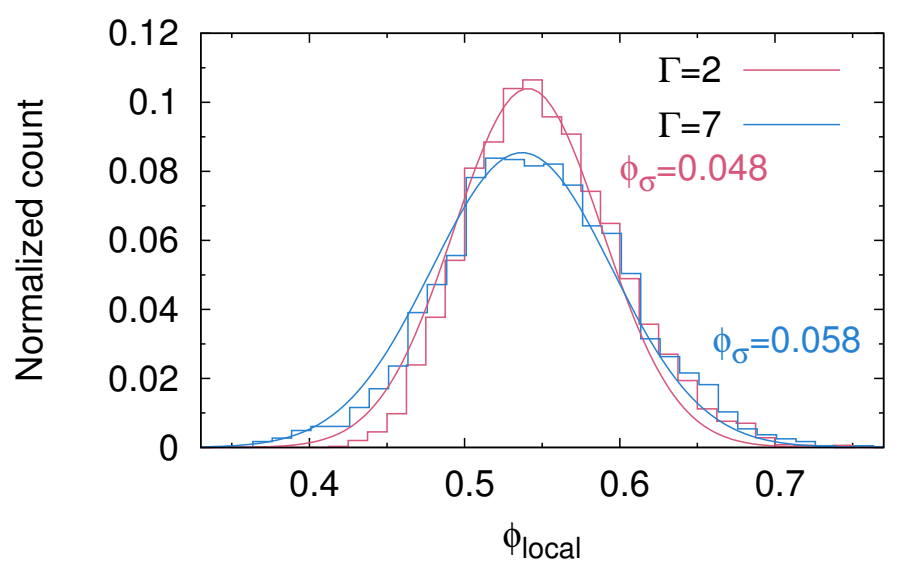

Figure 4.23: Distribution of $\phi_{\text {local }}$ for 400 taps at $\Gamma=2$ (red) and $10^{4}$ taps at $\Gamma=7$, with comparable global $\Phi=0.537$ resp. $\Phi=0.541$. The standard deviations of the fitted Gaussians are denoted by $\phi_{\sigma}$.

\subsubsection{Local contact types}

The mapping between local contact types and local packing fractions reveals which geometric configurations are preferred for a dense local packing. We assume that the maximum density of $\phi_{\text {local }}=1.0$ is obtained for one tetrahedron being covered on all faces by 4 perfectly aligned face-face contacts. On the other hand, a high number of point contacts is expected to leave larger voids and result in a smaller $\phi_{\text {local }}$ (consider the extremum, the lattice packing with $\Phi=0.36$ and 14 point contacts). This trend is visible in Fig. 4.24: face-face and edge-face contacts are more prevalent at higher packing fraction, on the cost of lower order point contacts. This trend looks more pronounced for the globally densest packings. It is noteworthy that the globally loose packing shows a substantial increase of the local constraint number from 11 to 14 , leveling off only at $\phi_{\text {local }} \approx 0.54$. The local packing fractions below 0.47 are probably close to mechanical instability, and the computed (average) numbers have larger errors due to the low number of occurrences. In the globally dense packing however, the local constraints increase only slightly from 15 to 16 , and saturate at a similar $\phi_{\text {local }} \approx 0.53$. This data is inconclusive to decide whether this packing fraction is associated with any physical meaning, e.g. a structural rearrangement, or within insignificant data scatter. For conclusive results, the significance of the datasets should be improved by averaging over a set of identically prepared packings at the same global $\Phi$.

An important question remains: What is the connection between the distribution of $Z\left(\phi_{\text {local }}\right)$ and the globally averaged $Z(\Phi)$ ? Intuitively, one 
assumes that the contact number of a single particle depends only on the local, but not on the global packing fraction. If this is the case, metrics like the face-face contact number $Z_{F F}$ or the constraint number $C$ should only vary with $\phi_{\text {local }}$ (resp. the Voronoi cell volume), but not with the global packing density the cells were picked from. A preliminary analysis did not confirm this hypothesis: for example, the number of contraints for selected values of $\phi_{\text {local }}$ depend also on the global $\Phi$. If that relation turns out to be robust, $\phi_{\text {local }}$ would be insufficient to characterize the distribution of contacts, and different values of $\Phi$ would imply also different local structures. A systematic variation of the "local" notion by spatial coarse-graining of $\phi_{\text {local }}$ towards $\Phi$ could possibly link to a cross-over distance to resolve this discrepancy.

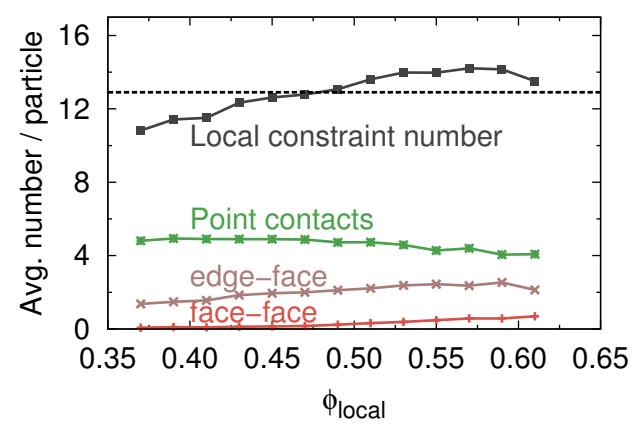

(a)

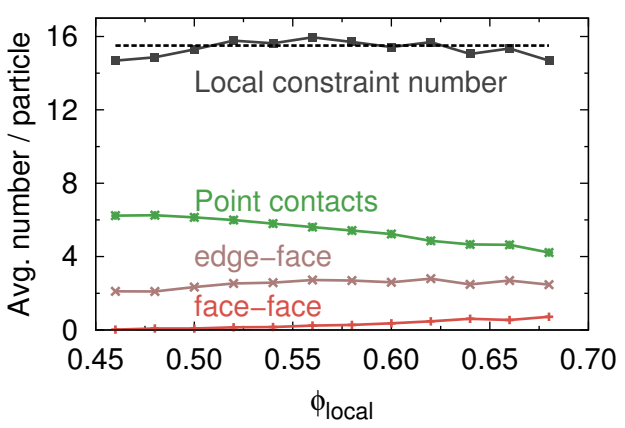

(b)

Figure 4.24: Local contact types vs. $\phi_{\text {local }}$ for (a) loose packing with global $\phi=.472$ (initial) (b) intermediate packing with global $\phi=.542$ (TAP). As a consequence of increasing face-face contacts, which cover a large part of the solid angle, the number of point contacts decreases.

\subsubsection{Local angle distributions}

Smith et. al.[80] have discussed angle distributions between faces by comparison to randomly arranged faces and edges. The probability of finding a random edge-face-contact with angle $\alpha_{E F}$ is weighted towards small alignment angles: $p\left(\alpha_{E F}\right) \sim \cos \left(\alpha_{E F}\right)$, and therefore expected to be ubiquituous in jammed packings of polyhedra. On the other hand, face-face contacts are expected to be very rare in random configurations, because the probability vanishes for ideal alignment: $p\left(\alpha_{F F}\right) \sim \sin \left(\alpha_{F F}\right)$. Note that $\alpha_{F F}$ denotes the angle between faces and is therefore $0^{\circ}$ for perfect alignment.

The experimental distribution of angles between "directed" face normals $\alpha_{f n}$ is depicted in Fig. 4.25 for a loose (initial) and a dense (VIB) sample. $\alpha_{f n}$ is computed for a pair of tetrahedra $q, l$ by choosing the pair of faces that are directed at each other, that is, enclosing the smallest angle with the distance vector $\vec{c}_{q}-\vec{c}_{l}$. Only local arrangements within an empirically chosen 
cutoff of $\left|\vec{c}_{q}-\vec{c}_{l}\right|<2.1 r_{\text {min }}$ are regarded for the distribution in Fig. 4.25. The sharp peak near $180^{\circ}$ corresponds to dimers (face-face contacts), which are more prominent in the dense samples than in the loose ones. During compaction of the packings, an additional broad peak emerges around the tetrahedral angle of $109.4^{\circ}$, setting in already at $\phi>0.52$. This peak can be clearly attributed to the formation of trimers (three tetrahedra face-face in a row), because the outer particles enclose this angle. Trimers have a centroid distance of $1.63 r_{\text {min }}$, which matches the shoulder in $F(r)$ (Fig. 4.14). Here, the complete angle distribution Fig. 4.25 offers more insight than $F(r)$ and is also easily interpreted with respect to geometric configurations.

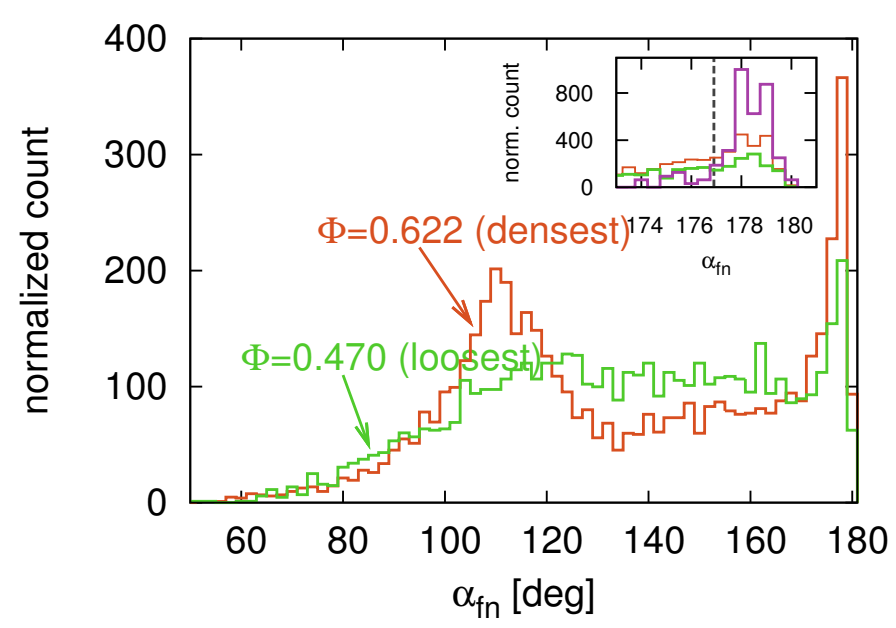

Figure 4.25: Distribution of angles between face normals $\alpha_{f n}$, restricted to particles within a centroid distance $r<2.1 r_{\min }$ (conjectured next-nearest neighbor shell). Inset shows a zoom-in of the acute angle region at $\alpha_{f n}>173^{\circ}$ : face-face calibration sample is overlayed (violett), the dotted line depicts the threshold for acceptance of face-face contacts.

\subsection{Comparison to DEM simulations}

L. Pournin developed an effective discrete element method (DEM) code, and performed simulations on spherotetrahedra, varying the particle sharpness, friction, and preparation protocol $[62,61]$. The following results are collaborative work: The packing configurations were kindly provided by Lionel Pournin, and I employed the CNS model for analysis of contact numbers and structures.

The model of a spherotetrahedron is defined by dilation (Sec. A.1) of a sphere with radius $r_{c}$ on a "skeleton" tetrahedron with circumradius (centroid-vertex distance) $l$, with the sharpness parameter $s=l / r_{c}$ (see Fig. 4.26 for illustration). The spherotetrahedron varies its shape smoothly from the sphere $(s=1)$ to the ideal geometric tetrahedron $(s=\infty)$. 


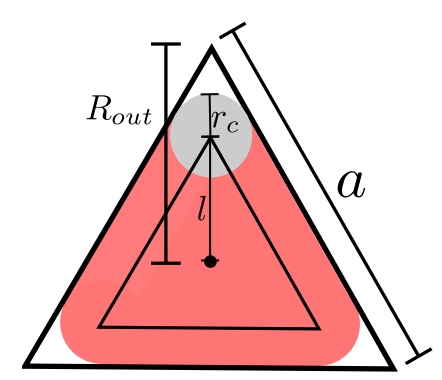

Figure 4.26: Geometric relations between side length $a$ of the spherotetrahedron, inradius $l$ of the skeleton, and corner curvature $r_{c}$ in the spherotetrahedron model (2D sketch).

The physical tetrahedra particles are characterized by the "outer" side length $a$ and the corner curvature $r_{c}$. These parameters are related by $r_{c}=R_{\text {out }} /(s+2)$, where $R_{\text {out }}=3 a /(2 \sqrt{6})$ is the circumradius of the outer tetrahedron with sidelength $a$. Thus, the physical tetrahedra with side length $a=7 \mathrm{~mm}$ and corner curvature $r_{c}=0.15-0.20 \mathrm{~mm}$ are approximated best by spherotetrahedron models with sharpness $s=19-26$. The relevant simulations performed by L. Pournin comprise three datasets with $s=[9,20,30]$ and friction $\mu$ being either 0.4 or 0.8 , as compiled in Tab. 4.2.

For the presented protocols, spherotetrahedra are poured into a cylindrical container under the influence of gravity, either quickly (duration: $2 \mathrm{~s}$ ) or slowly (duration $12 \mathrm{~s}$ ). Optionally, constant vertical vibration is applied during fast pouring. In contrast to typical MC simulations, these simulations agree with our experimental protocol closely, particularly with the initial preparation and the VIB protocol. In a range of parameters comparable to experiments, packing fractions between 0.61 and 0.71 are reproduced. This allows also to validate the model-based contact number counting against an independent set of simulation data. As Tab. 4.2 shows, contact numbers obtained for the DEM data agree with the experimental contact numbers. Furthermore, the width of the CNS step, as measured by $\sigma_{C N S}$ (see Sec. 3.2), increases with the corner radius (resp. inverse sharpness), as expected from the model. It is remarkable that the contact numbers seem to saturate near $Z=9$ for $\phi$ as high as 0.637 , which supports the experimental obser-

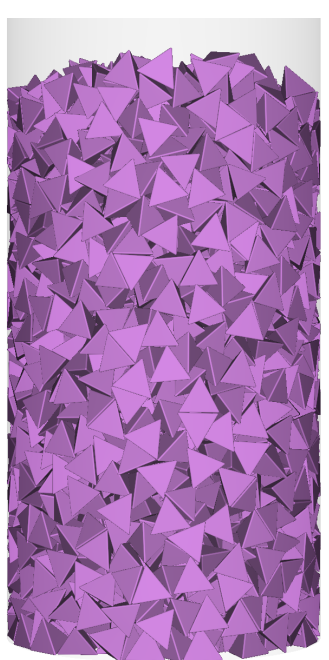

Figure 4.27: Packing of spherotetrahedra, friction $\mu=0.4$, sharpness $\sigma=20$ vation up to $\phi=0.622$ (see Fig. 4.8). Moreover, even if the DEM packings are slightly denser than the experimental packings, contact numbers are in excellent accordance with the highest values of $Z \approx 8.8$. Note that these 
values coincide remarkably well with the Jamming threshold from simulations on frictionless tetrahedra [79], where $\Phi_{J}=0.61$ and $Z_{J}=8.6$ was determined. These results suggest that there an universal upper limit for $Z$ exists, which is independent of simulation or experimental details. Thus, a purely geometric argument (volume exclusion) is the most likely explanation. For example, each face of a tetrahedron could be covered by a dimer in an edge-face contact geometry, which yields $Z=8$ and a constraint number of $C=8 \cdots 2.5=20$. However, in lack of deeper insight from computational geometry, this question remains presently open.

Another notable consequence is that the CNS model can be applied to numerically created packings, and it is superior to an arbitrary threshold for contact acceptance. Fig. 4.28 depicts the CNS fits corresponding to the contact numbers in Tab. 4.2.

\begin{tabular}{cccccc}
\hline$\Phi$ & friction $\mu$ & sharpness $s$ & radius $r_{c}[\mathrm{~mm}]$ & $\mathrm{Z}$ & $\sigma_{C N S}$ \\
\hline \hline .617 & 0.4 & 30 & .23 & 8.6 & .09 \\
.636 & 0.8 & 9 & .80 & 6.8 & .21 \\
.637 & 0.4 & 20 & .35 & 8.5 & .12 \\
\hline Tetrahedra: .622 & 0.8 & 26 & .15 & 8.9 & .22
\end{tabular}

Table 4.2: Parameters of selected spherotetrahedra packings in DEM simulations[61]: Packing fraction $\Phi$, friction $\mu$, sharpness $s$ and corner curvature equivalent to our physical tetrahedra; resulting contact number $Z$ computed via CNS model and accuracy of model $\sigma_{C N S}$.

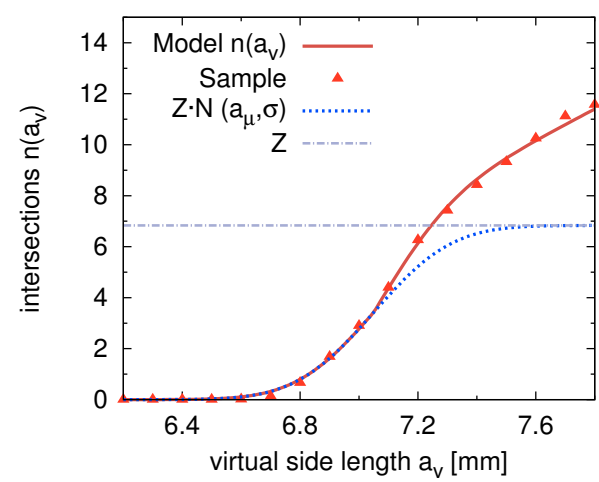

(a)

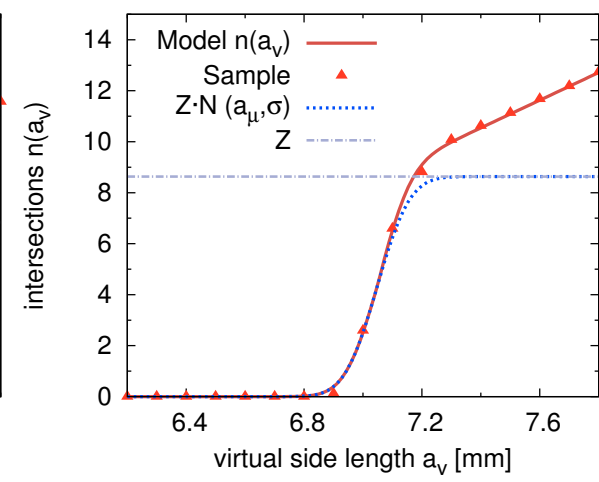

(b)

Figure 4.28: Counting contacts of spherotetrahedra packings with the CNS model. (a) For low sharpness $(s=9)$, the step is much broader $\left(\sigma_{C N S}=0.21\right)$ than for (b) high sharpness $(s=30)$, where $\sigma_{C N S}=0.09$. 


\section{Chapter 5}

\section{Packings of octahedra and spaghetti}

This chapter summarizes work that has been done in collaboration with co-workers.

\section{$5.1 \quad$ Octahedra}

This work was done in collaboration with Nirmal N. Thyagu. In addition to tetrahedra packings, also octahedra particles with $7 \mathrm{~mm}$ side length were analysed by tapping experiments and tomography. In contrast to tetrahedra, octahedra do readily crystallize when packings are prepared by the VIB procotol or by long tapping experiments. Crystallization is heterogenous, that is, it proceeds from the bottom and from the cylinder walls. Fig. 5.2 shows different views of a reconstructed Octahedra (VIB) packing, illustrating the competing ordering effects from bottom and side walls (coloring according to orientation).

A mixture of octahedra and tetrahedra can form a space filling crystal. Crystallization emerges layer-wise, when particles are mixed in the appropriate ratio of 1:2 (octahedra:tetrahedra), and poured in under constant vibration (using any protocol similar to VIB). If the mixture is first filled in and then continuously tapped, size segregation and convection rolls appear, which inhibit layer-wise crystallization, as Fig. 5.3 illustrates. Octahedra aggregate in the upper part, and tetrahedra seem to upwell in the middle of the container. 


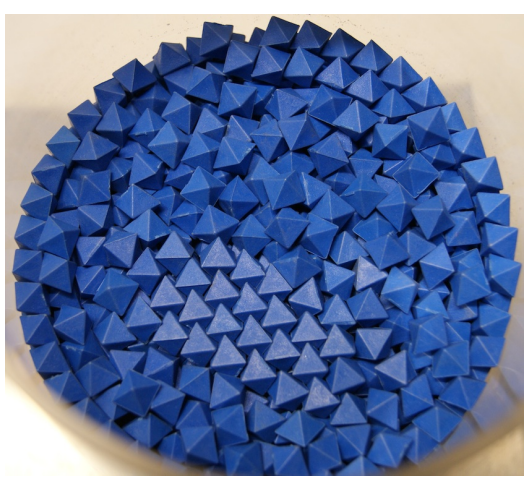

(a)

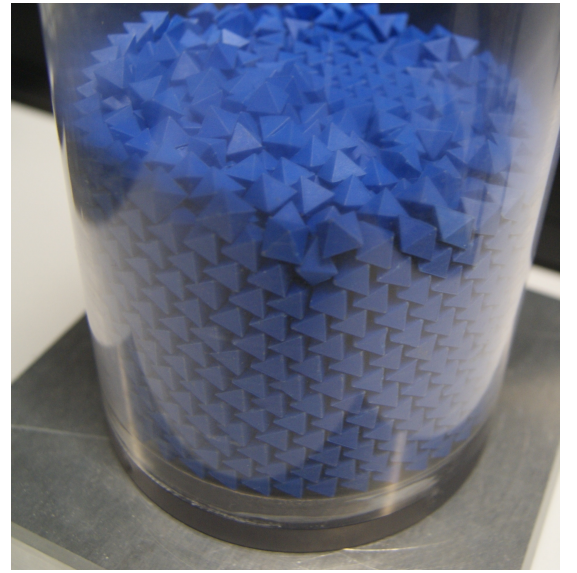

(b)

Figure 5.1: Dense packing of octahedra particles (a) Photograph of partly crystalline surface of octahedra VIB packing (b) Crystallization from the wall

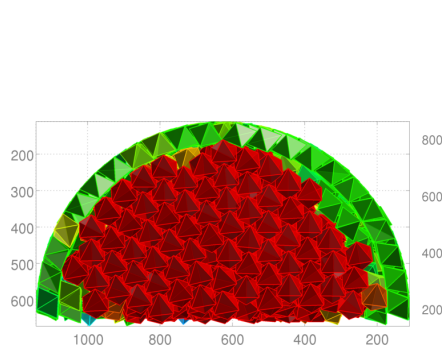

(a)

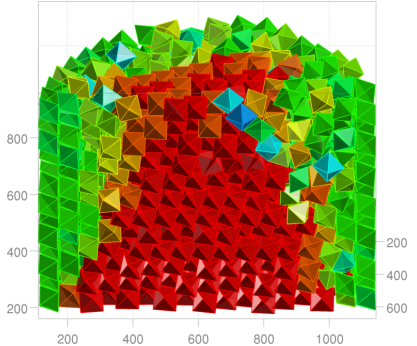

(b)

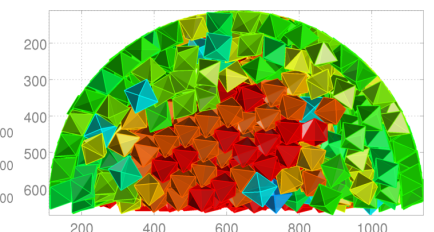

(c)

Figure 5.2: Reconstructed octahedra particles (VIB protocol), colored by orientation: Maximum alignment of a face towards the bottom plane $=$ red, minimum $=$ blue. (a) Bottom view: Crystallization of faces towards the cylinder bottom (b) Side view, half-cut: Crystallization from the bottom decays towards the top (c) Top view 


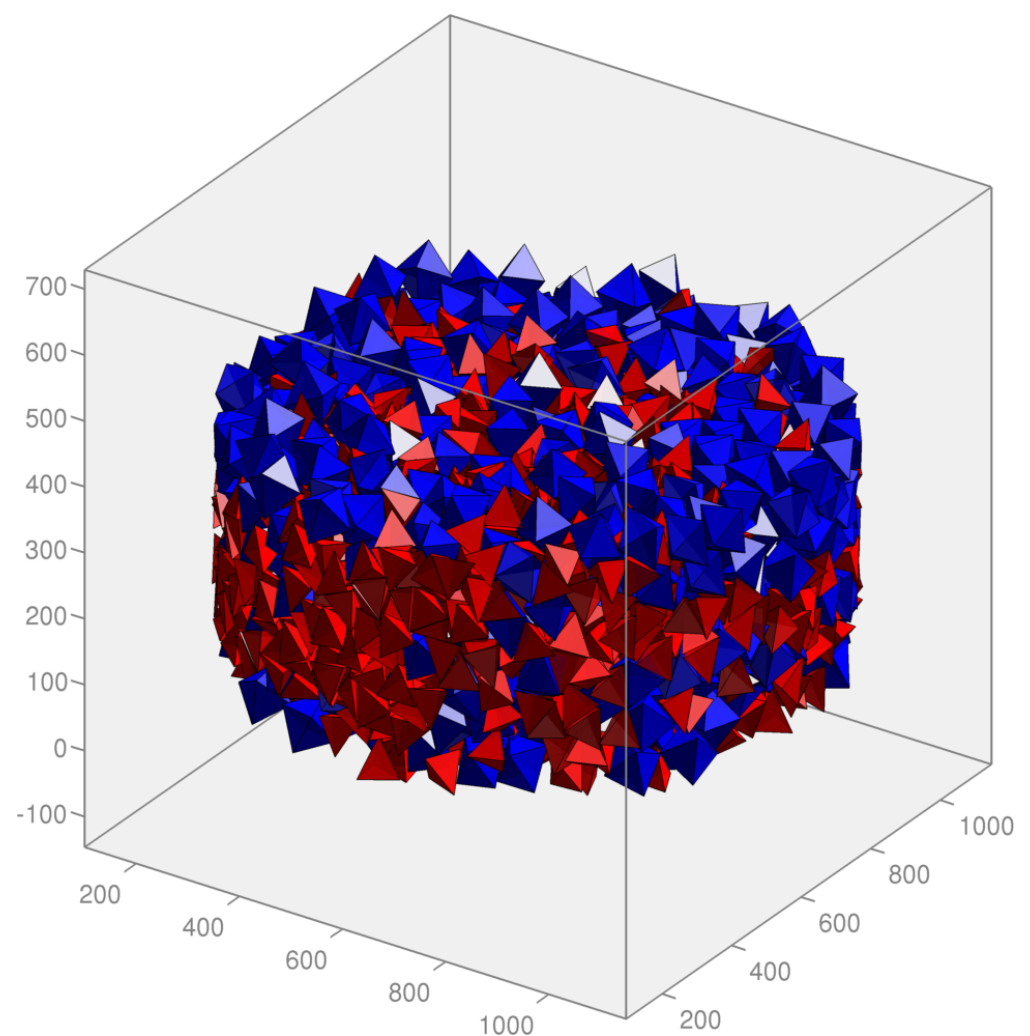

Figure 5.3: Observed size segregation of a tetrahedra-octahedra mixture (tetrahedra colored red, octahedra blue). 


\section{$5.2 \quad$ Spaghetti packings}

The idea to investigate the packing properties of spaghetti as a model for rods or filaments originates from Claus Heussinger and my supervisor Matthias Schröter. From 2011 to 2013, I supervised Sebastian Pitikaris (BSc thesis), Christian Brosowski (BSc thesis), both at the Theory department of the Faculty of Physics, Göttingen, and Cyprian Lewandowski from Imperial College, London (RISE exchange program), and assisted in sample preparation, tomographic acquistion, particle detection and further analysis. Here, only a brief overview of the experiment is given and selected results are highlighted; for further details the reader is referred to the respective BSc theses. Additionally, Cyprian developed a Matlab interface for $3 \mathrm{D}$ visualizaion with Povray and Blender, with a detailed documentation [43].

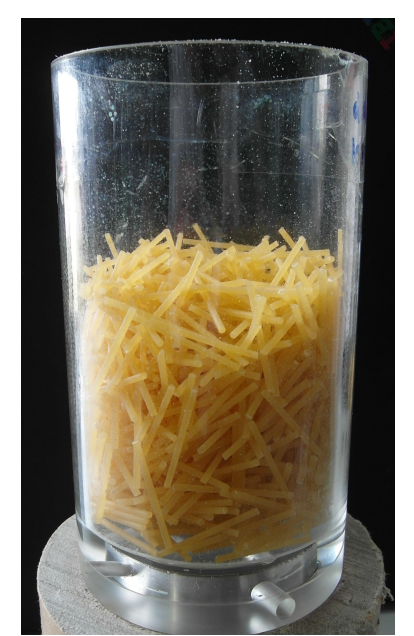

Figure 5.4: Experimental setup: Packing of spaghetti (rods) in a cylinder.

\subsubsection{Motivation}

Philipse [59] predicted a universal scaling law for the random packing of rods with length $L$ and diameter $D$. According to the model, the contact number $Z$ scales with packing fraction $\Phi$ and the aspect ratio $L / D$, that is:

$$
Z \sim \Phi \frac{L}{D}
$$

The tomography of spaghetti packings was chosen as an experimental model system for verifying this theory. Technical prerequisites are an accurate particle detection algorithm and the accurate counting of contact numbers, which is achieved here with a modified CNS model.

\subsubsection{Preparation}

Barilla Spaghettini (Diameter $D=1.39 \mathrm{~mm}$ ) were cut to different length $(L=2,3,4 \mathrm{~cm})$ using a common paper-cutter. The cut pieces were filled in with various techniques (e.g. using a rough bottom), in order to minimize alignment effects. The main experimental challenge is to create a disordered packing, where alignment effects from walls can be minimized. 


\subsubsection{Particle detection}

As mentioned in Sec. 3.1.5, the task of registering particles with pronounced shape anisotropy can be solved via a singular value decomposition (SVD). This is a factorization of a transformation matrix $\mathbf{M}$, decomposed into a sequential rotation $\mathbf{V}$, a diagonal scaling $\mathbf{S}$ and another rotation $\mathbf{U}$, so that $\mathbf{M}=\mathbf{U S V}^{T}$. Fig. 5.5a illustrates the decomposition by transforming a unit circle into a rotated and sheared ellipse. Here, the largest eigenvalue $\sigma_{1}$ is the half-axis of the ellipse, and the eigenvector $\vec{u}_{1}$ is aligned with the principal axis.

Fig. 5.5b shows the result of applying the SVD successfully for registration of a arbitrarily oriented spaghetti in the packing.

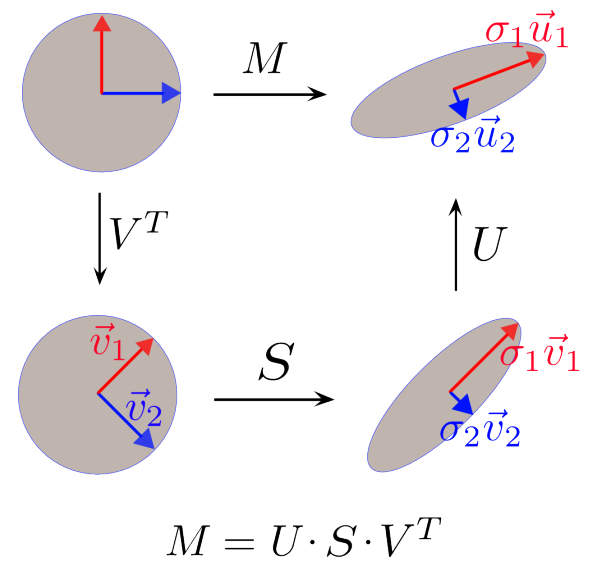

(a)

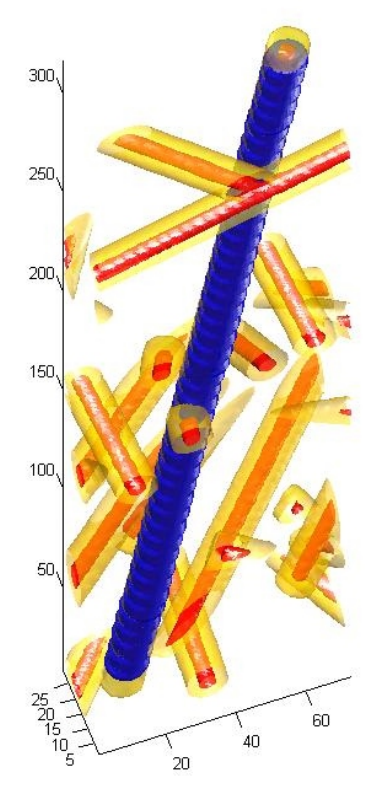

(b)

Figure 5.5: (a) Illustration of SVD decomposition; (b) Registration of rod packings (tomograph of Barilla Spaghettini): binarized foreground particles (yellow); erosion operator applied for segmentation (red); SVD applied on eroded image and reconstructed by largest eigenvalue $\max \left(\sigma_{i}\right)$

\subsubsection{Analysis}

The direction vector from the SVD analysis Fig. 5.5b is a direct metric for the orientational alignment, as Fig. 5.6 illustrates.

Preliminary results on the verification of the Philipse scaling law (Eq. 5.1) look promising: With increasing aspect ratio $L / D$, the ratio of $Z / \Phi$ increases proportionally. The deviations from the scaling model are mainly 


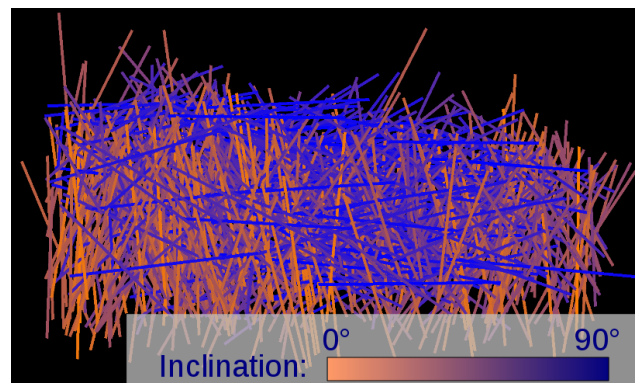

Figure 5.6: Orientation of spaghetti particles according to their azimuthal angle (inclination): Prevalence of vertical alignment

attributable to orientational alignment effects from the walls. The tendency for vertical alignment during tapping is also observed in DEM simulations on spherocylinders [63].

Besides the contact number, also Voronoi volume distributions of spaghetti packings can be examined. Fig. 5.7 shows an exemplary set of spaghetti with their associated Voronoi volumes.

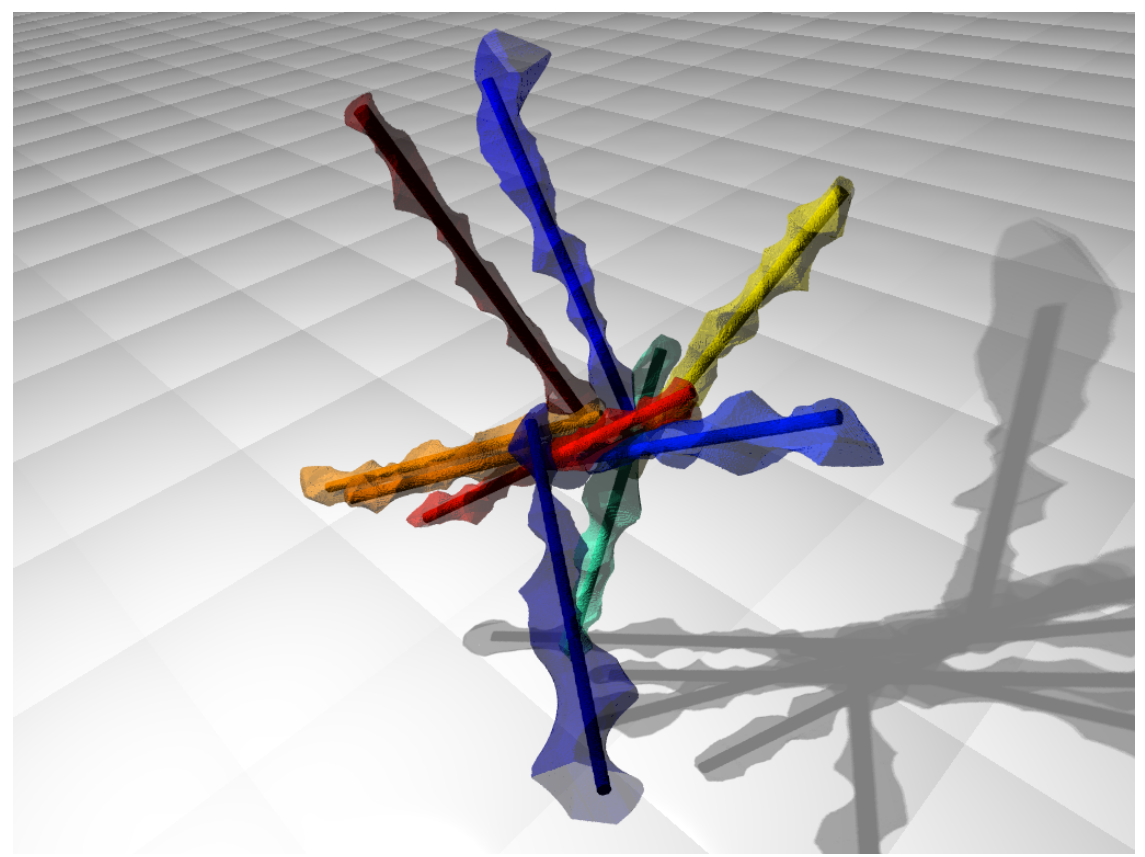

Figure 5.7: Selected spaghetti with their associated Voronoi volumes in the core region of a packing. Rendered using POVRay, coloring from blue (largest volume) to red (lowest volume) 


\section{Chapter 6}

\section{Conclusion and Outlook}

\subsection{Tetrahedra packings are hyperstatic}

In this study, the geometric structure of frictional, disordered tetrahedra packings was analysed in detail. The choice of the granulate was motivated by moving away from the "spherical cow" paradigm towards more realistic models.

In the first part, we showed that different preparation protocols can be utilized to create packings with a wide range of packing fractions $(\Phi=0.47 \ldots 0.62)$. A comparison between the annealing procedure RAMP and tapping with varying intensity $\Gamma$ revealed that the steady state density $\Phi_{\infty}$ is very likely history independent and negatively correlated with $\Gamma$.

The spatial distributions of packing fractions showed that gentle tapping causes a more homogenous distribution than vigourous shaking. Moreover, the VIB protocol exhibits the highest packing fraction among all samples.

The second part tackled mechanical stability in the context of the Jamming paradigm. The generalized constraint number $C$, which depends on the contact geometries, was found to reach values between 12 and 18, substantially larger than the isostatic limit of 6 constraints per particle. Experimentally prepared, frictional tetrahedra packings are therefore hyperstatic, in contrast to previous studies claiming isostaticity [34, 85]. Depending on the protocol, packings with the same $\Phi$, but different contact number $Z$ can exist. The distribution of the number of face-face contacts was found to follow a random process with a sample-dependent probability, which increases with higher packing fraction. The break-down of the random, uncorrelated probability was suggested as a possible distinction criterion between disorder and order.

Subsequently, we addressed the question of order or disorder in tetrahedra packings. To this aim, we employed the translational and orientational correlation functions $g(r)$ and $F(r)$, and discussed the applicability of different order metrics. Since sensitivity to structural features was insufficient, 
we turned to more specific metrics based on face-face contact numbers. The outcome was that a) dense local structures have a abundance of face-face contacts, b) face-face-connected clusters grow strongly with increasing $\Phi$, and c) the face-face contact number suggests itself as an order parameter which is superior to the participation ratio [76].

On a local level, the distributions of local Voronoi volumes (effective volumes) were computed and compared to Gaussian and Gamma distribution models. It turned out that both distributions have limitations, rooted in the model assumptions, that cause a mismatch between observed volumes and model distributions.

The mapping of local contact types to $\phi_{\text {local }}$ revealed that increasing global packing fraction is locally realised by point contacts with low $\phi_{\text {local }}$ turning into edge-face or face-face contacts with higher $\phi_{\text {local }}$.

Results contained in this thesis were published in Ref. [52], and the complete packing configurations are available for download at the dryad repository [51].

A collaboration with Nirmal N. Thyagu covered preparation and analysis of octahedra packings, where a interesting crystallization behaviour could be observed. Tetrahedra-Octahedra mixtures showed size segregation (TAP protocol) or partly crystallization (VIB). A second collaboration with C. Heussinger dealt with packings of rods (Spaghetti), which showed the broad applicability of the CNS method, and even a quantitative agreement with the proposed scaling law for the contact number.

\subsection{Outlook}

A key question to be addressed in future work is the transition between order and disorder. To this aim, experimental protocols for creating denser packing structures are needed. Cyclic shear has been successfully applied to surpass the random close packing limit for sphere packings [54], and should be transferable to tetrahedra. Currently, preliminary results on octahedra in a shear cell are acquired by my colleague Nirmal N. Thyagu. The preparation of a quasi-crystal might be possible if a seed layer is constructed at the bottom (private communication with P. Damasceno). Dense packings could also be created with addition of attractive forces, like adding water with a wetting agent.

Regarding the TAP protocol, the dynamics of the pore space could be analysed and compared to the compaction of spheres, which exhibit an exponential decay of pore volumes [68]. Computationally, the pore space could be computed from a tomography as follows: Binarize the tomogram, compute the Euclidean distance transform on the background phase, and extract pore the radii directly from the value of the local maxima.

The question how contacts or constraints depend on the local and/or 
global scale of packing fractions is not answered conclusively. Further analysis should address the influence of spatial coarse-graining and perform more ensemble averages for a given packing fraction.

The collaboration with L. Pournin should be continued, as both model, preparation technique and results compare well to the present experiment, and a possible extension to other preparation techniques or particle shapes is straightforward.

In addition, I hope that the publication of the packing configurations on the dryad repository [51] facilitates further progress in the field of disordered packings of non-spherical particles. 


\section{Appendix A}

\section{Image processing}

\section{A.1 Morphological image processing}

The reconstructed tomographic volume is a three-dimensional grey value image $I$, where $l_{\max }$ discrete grey-levels are observed at $\mathbf{x} \in \mathbb{Z}^{3}$ discrete coordinates. In analogy to pixels (picture elements) in a $2 \mathrm{D}$ image, the discrete coordinates in 3D images are termed voxels (volume elements).

A binary image is defined as $I \mapsto\{0,1\}$, so that a voxel either belongs to a background (0) or foreground (1) segment. The corresponding inverted image is denoted by $\bar{I}$ and maps the values $\{0,1\}$ to $\{1,0\}$, which is equivalent to a elementwise logical NOT. If $I$ and $J$ are two binary images, the elementwise union and intersection operators $\cup$ and $\cap$ are defined as [81]:

$$
\begin{array}{r}
(I \cup J)(\mathbf{x})=\max [(I(\mathbf{x}), J(\mathbf{x})] \\
(I \cap J)(\mathbf{x})=\min [(I(\mathbf{x}), J(\mathbf{x})]
\end{array}
$$

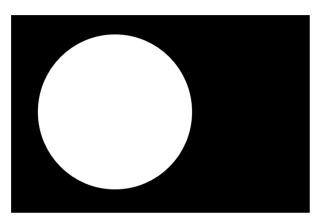

(a) Binary image $I$

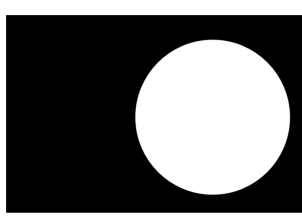

(b) Binary image $J$

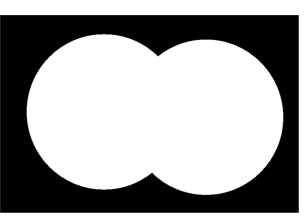

(c) $I \cup J$

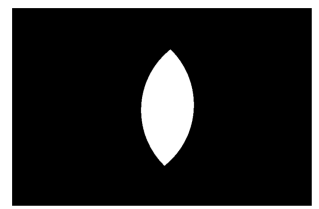

(d) $I \cap J$

Figure A.1: Elementary set operators on images

Here the basic concepts and operations of morphological image processing, which are relevant in the image processing workflow, are outlined.

1. The dilation of the image $I$ by the structure element $S$ is the Minkowski addition between the two sets: $I \oplus S$.

2. The erosion of the image $I$ by $S$ is the corresponding Minkowski subtraction: $I \ominus S$.

Fig. A.2 illustrates the result of dilation and erosion with a disk-shaped structuring element: 


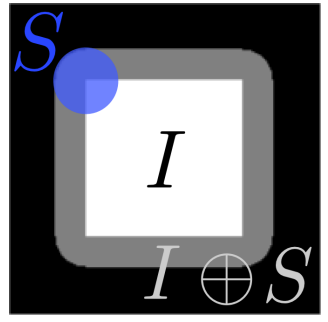

(a)

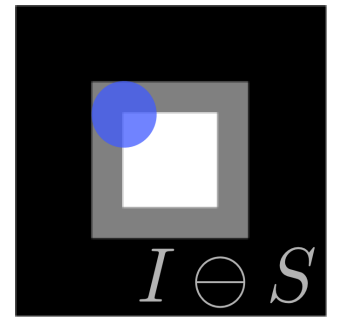

(b)

Figure A.2: Dilation and erosion operators. (a) Dilation: White square $I$ dilated by the blue disk $S$ results in the larger grey rounded square $I \uplus S$ (b) Erosion: Large grey square $I$ eroded by the blue disk $S$ results in the smaller white square.

\section{A.2 Data structures}

Internally, the platonic bodies with $v$ vertices, $f$ faces and $e$ edges per face are represented by the following data structure: Vertex Coordinates and other particle specific properties are stored in the first hierarchic layer. In the second layer, for each face, the indices of the corresponding vertices (e.g. 1,3,4) and edges are stored (linked list). This structure is easily accessible for the reader and appropriate for execution of geometric queries (adapted from http://www.qhull.org).

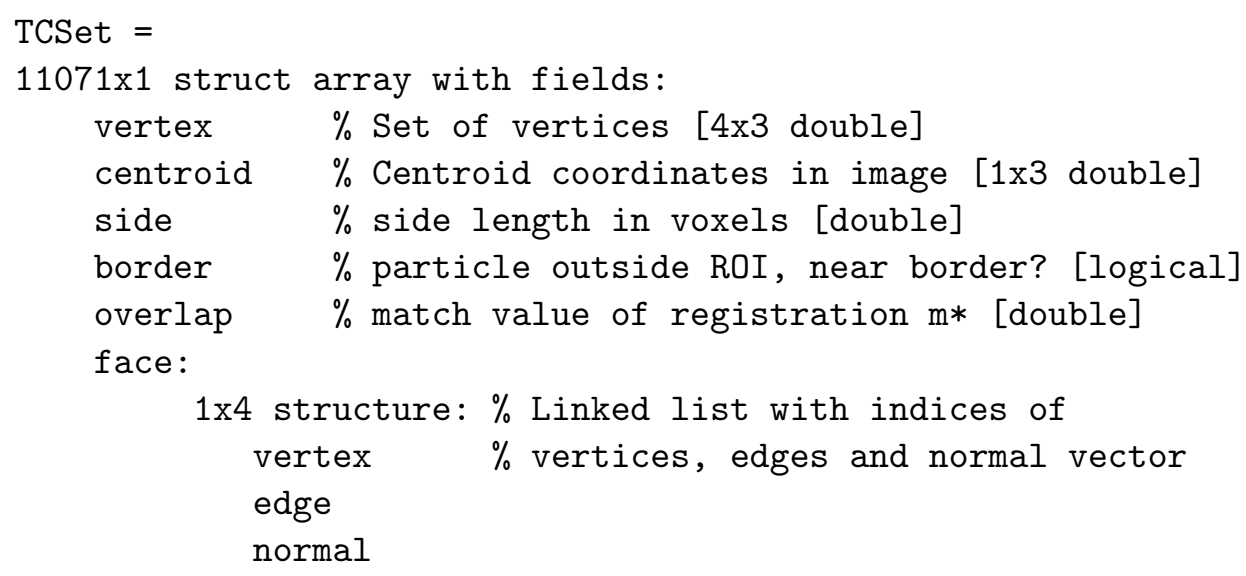

Each face is associated with its 3 vertices, the 3 edges and the face normal vector (pointing outwards).

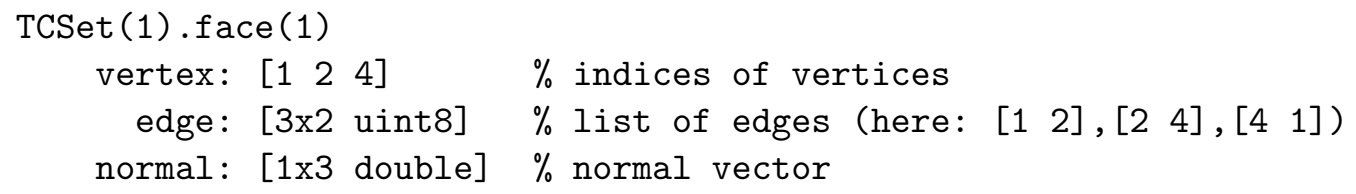




\section{Appendix B}

\section{Neudecker vs. Jaoshvili}

The following table B.1 shows a comprehensive side-by-side comparison between this study and the single comparable experiment by Jaoshvili et. al.[34]. In particular, the smaller tetrahedral particles, higher resolution of X-ray CT, and analysis via the physically motivated CNS function allow for a much more accurate and detailed analysis. All measured properties (range of $\phi, Z, Z_{F F}, C$ ) differ strongly between these experiments. 


\begin{tabular}{|c|c|c|}
\hline Author & Jaoshvili[34] & This work \\
\hline \multicolumn{3}{|l|}{ Particles } \\
\hline Slice view & & \\
\hline $\begin{array}{l}\text { Side length } a \\
\text { Corner radius } \\
\text { (as } \% \text { of } a \text { ) }\end{array}$ & $\begin{array}{l}20 \mathrm{~mm} \\
r=1.5 \mathrm{~mm}(7-8 \%)\end{array}$ & $\begin{array}{l}7 \mathrm{~mm} \\
r=0.15 \mathrm{~mm}(2-3 \%)\end{array}$ \\
\hline Friction & $\mu=0.22 \pm 0.04$ & $\mu=0.87 \pm 0.03$ \\
\hline Preparation & $\begin{array}{l}\text { particles sequentially } \\
\text { added }+ \text { shaken }\end{array}$ & $\begin{array}{l}4 \text { different preparation } \\
\text { methods }\end{array}$ \\
\hline Exp. Setup & MRI @ 0.5mm/voxel & $\begin{array}{l}\text { X-ray CT @ } \\
0.1 \mathrm{~mm} / \text { voxel }\end{array}$ \\
\hline Statistics & 311 particles & $\begin{array}{l}>4000 \text { particles in each } \\
\text { sample }\end{array}$ \\
\hline Packing fraction & $\begin{array}{l}\phi=[0.65 \ldots 0.74] \\
\text { (only } 1 \text { sample analysed) }\end{array}$ & $\begin{array}{l}\phi \in[0.469 \ldots 0.622](22 \\
\text { samples })\end{array}$ \\
\hline $\begin{array}{l}\text { Contact } \\
\text { numbers }\end{array}$ & $Z=6.3 \pm 0.5$ & $Z \in[6.6 \ldots 9.0]$ \\
\hline $\begin{array}{l}\text { Face-face } \\
\text { contacts }\end{array}$ & $Z_{F F}=2.2$ & $Z_{F F} \in[0.2 \ldots 0.7]$ \\
\hline $\begin{array}{l}\text { Constraints } \\
\text { (per particle) }\end{array}$ & $C_{\text {frictionless }}=6.0 \pm 0.8$ & $\begin{array}{l}C_{\text {frictionless }} \in[4.3 \ldots 6.6] \\
C_{\text {frictional }} \in[12 \ldots 18]\end{array}$ \\
\hline
\end{tabular}

Table B.1: Direct comparison between tomographic analysis of tetrahedral dice packing[34] and this work. 


\section{Appendix C}

\section{Variable listing}

$a$

$\alpha_{F F}$

$\alpha_{f n}$

C

$\Phi$

$\phi_{\text {local }}=V_{\text {tet }} / V$

$R_{\text {in }}$

$R_{\text {out }}$

V

$V_{\text {tet }}$

$V_{\text {oct }}$

Z

$Z_{F F}, Z_{E F}, Z_{V F}, Z_{E E}$

ROI

Voxel side length of particle

Angle between two tetrahedra faces (in degree)

Angle between two tetrahedra face normals (in degree)

Generalized constraint number per particle

globally averaged packing fraction

local packing fraction

Inradius of tetrahedron or octahedron: touches all faces

Circumradius: passes all vertices

Voronoi volume of particle

Volume of tetrahedron

Volume of octahedron

Contact number (per particle)

Number of face-face, edge-face,vertex-face and edge-edge contacts (per particle)

Region Of Interest, sub-volume used for analysis

Coordinate in $3 \mathrm{D}$ with assigned intensity value 


\section{Bibliography}

[1] Arun KS, Huang TS, and Blostein SD. Least-squares fitting of two 3-d point sets. IEEE Transactions on Pattern Analysis and Machine Intelligence, 9(5):698, 1987.

[2] Aste T and Di Matteo T. Emergence of gamma distributions in granular materials and packing models. Phys. Rev. E, 77(2):021309, 2008.

[3] Aste T, Saadatfar M, and Senden TJ. Geometrical structure of disordered sphere packings. Phys. Rev. E, 71:061302, 2005. doi: 10.1103/PhysRevE.71.061302.

[4] Athanassiadis AG, Miskin MZ, Kaplan P, Rodenberg N, Lee SH, Merritt J, Brown E, Amend J, Lipson H, and Jaeger HM. Particle shape effects on the stress response of granular packings. arXiv preprint arXiv:1308.2241, 2013.

[5] Atman A, Brunet P, Geng J, Reydellet G, Combe G, Claudin P, Behringer RP, and Clment E. Sensitivity of the stress response function to packing preparation. Journal of Physics: Condensed Matter, 17(24):S2391, 2005. URL http://stacks.iop.org/0953-8984/17/i= $24 / \mathrm{a}=002$.

[6] Baker J and Kudrolli A. Maximum and minimum stable random packings of platonic solids. Phys. Rev. E, 82:061304, 2010. doi: 10.1103/PhysRevE.82.061304.

[7] Baranau V, Hlushkou D, Khirevich S, and Tallarek U. Pore-size entropy of random hard-sphere packings. Soft Matter, 9:3361, 2013.

[8] Bernal J and Mason J. Packing of spheres: co-ordination of randomly packed spheres. Nature, 188, 1960.

[9] Bernal JD. Geometrical approach to the structure of liquids. Nature, 183(4655):141-147, 1959. ISSN 0028-0836.

[10] Buzug TM. Einführung in die Computertomographie. Springer Berlin Heidelberg, 2004. 
[11] Cambau T, Hure J, and Marthelot J. Local stresses in the janssen granular column. Phys. Rev. E, 88:022204, 2013. doi:10.1103/PhysRevE. 88.022204. URL http://link.aps.org/doi/10.1103/PhysRevE. 88. 022204.

[12] Chen E, Engel M, and Glotzer S. Dense crystalline dimer packings of regular tetrahedra. Discrete \& Computational Geometry, 44:253-280, 2010. 10.1007/s00454-010-9273-0.

[13] Chen ER. A dense packing of regular tetrahedra. Discrete \& Computational Geometry, 40(2):214, 2008.

[14] Ciamarra MP, Richard P, Schroter M, and Tighe BP. Statistical mechanics for static granular media: open questions. Soft Matter, 8:97319737, 2012. doi:10.1039/C2SM06898B. URL http://dx.doi.org/10. 1039/C2SM06898B.

[15] Conway JH and Torquato S. Packing, tiling, and covering with tetrahedra. Proceedings of the National Academy of Sciences, 103(28):10612, 2006 .

[16] Cuisenaire O. Distance transformations: fast algorithms and applications to medical image processing. Ph.D. thesis, Belgique: Universite Catholique de Louvain, 1999.

[17] Damasceno PF, Engel M, and Glotzer SC. Crystalline assemblies and densest packings of a family of truncated tetrahedra and the role of directional entropic forces. ACS Nano, 6:609614, 2012.

[18] Donev A, Cisse I, Sachs D, Variano EA, Stillinger FH, Connelly R, Torquato S, and Chaikin PM. Improving the density of jammed disordered packings using ellipsoids. Science, 303(5660):990-993, 2004.

[19] Donev A, Connelly R, Stillinger FH, and Torquato S. Underconstrained jammed packings of nonspherical hard particles: Ellipses and ellipsoids. Phys. Rev. E, 75:051304, 2007. doi:10.1103/PhysRevE.75.051304.

[20] Ediger MD. Spatially heterogeneous dynamics in supercooled liquids. Annual review of physical chemistry, 51(1):99, 2000.

[21] Edwards SF and Oakeshott R. Theory of powders. Physica A: Statistical Mechanics and its Applications, 157(3):1080, 1989.

[22] Feldkamp L, Davis L, and Kress J. Practical cone-beam algorithm. JOSA A, 1(6):612, 1984.

[23] Friedman JH, Bentley JL, and Finkel RA. An algorithm for finding best matches in logarithmic expected time. ACM Transactions on Mathematical Software (TOMS), 3(3):209, 1977. 
[24] Gold S, Rangarajan A, Lu CP, Pappu S, and Mjolsness E. New algorithms for $2 \mathrm{~d}$ and $3 \mathrm{~d}$ point matching:: pose estimation and correspondence. Pattern Recognition, 31(8):1019, 1998.

[25] Haji-Akbari A, Engel M, and Glotzer S. Phase diagram of tetrahedra. J. Chem. Phys., 2011.

[26] Haji-Akbari A, Engel M, Keys AS, Zheng X, Petschek RG, PalffyMuhoray P, and Glotzer SC. Disordered, quasicrystalline and crystalline phases of densely packed tetrahedra. Nature, 462:773, 2009.

[27] Hales TC. Sphere packings, i. page 379, 2011.

[28] Halsey TC and Ertaş D. A ball in a groove. Physical Review Letters, 83(24):5007, 1999.

[29] Henkes S, van Hecke M, and van Saarloos W. Critical jamming of frictional grains in the generalized isostaticity picture. Europhysics Letters, 90(1):14003, 2010.

[30] Hilbert D. Mathematische Probleme, Vortrag gehalten auf dem internationalen Mathematiker-Kongreß zu Paris. Nachr. Ges. Wiss. Göttingen, page 253, 1900 .

[31] Hough PV. Method and means for recognizing complex patterns. 1962. US Patent 3,069,654.

[32] Jaeger HM, Nagel SR, and Behringer RP. Granular solids, liquids, and gases. Reviews of Modern Physics, 68(4):1259, 1996.

[33] Janssen H. Versuche über Getreidedruck in Silozellen. VDI Bd, 39:1045, 1895.

[34] Jaoshvili A, Esakia A, Porrati M, and Chaikin PM. Experiments on the random packing of tetrahedral dice. Phys. Rev. Lett., 104:185501, 2010.

[35] Jerkins M, Schröter M, Swinney HL, Senden TJ, Saadatfar M, and Aste T. Onset of mechanical stability in random packings of frictional spheres. Phys. Rev. Lett., 101:018301, 2008. doi:10.1103/PhysRevLett. 101.018301. URL http://link.aps.org/doi/10.1103/PhysRevLett. 101.018301.

[36] Jiao Y, Stillinger FH, and Torquato S. Nonuniversality of density and disorder in jammed sphere packings. Journal of Applied Physics, 109(1):013508, 2011. 
[37] Jiao Y and Torquato S. Maximally random jammed packings of platonic solids: Hyperuniform long-range correlations and isostaticity. Phys. Rev. E, 84:041309, 2011. doi:10.1103/PhysRevE.84.041309.

[38] Kallus Y and Elser V. Dense-packing crystal structures of physical tetrahedra. Phys. Rev. E, 83(3):036703, 2011.

[39] Kallus Y, Elser V, and Gravel S. Dense periodic packings of tetrahedra with small repeating units. Discrete $\& 5$ Computational Geometry, 44:245-252, 2010.

[40] Keys AS, Iacovella CR, and Glotzer SC. Characterizing complex particle morphologies through shape matching: Descriptors, applications, and algorithms. Journal of Computational Physics, 230(17):6438, 2011.

[41] Kroon DJ. FMINLBFGS: Fast limited memory optimizer. http://www . mathworks.com/matlabcentral/fileexchange/ 23245-fminlbfgs-fast-limited-memory-optimizer. Updated 2010-11-02.

[42] Lapp T, Rohloff M, Vollmer J, and Hof B. Particle tracking for polydisperse sedimenting droplets in phase separation. Experiments in fluids, 52(5):1187, 2012.

[43] Lewandowski C. ExportVoxelData. http://www.mathworks.com/ matlabcentral/fileexchange/43640-export-voxel-data.

[44] Liu A and Nagel S. Nonlinear dynamics: Jamming is not just cool any more. Nature, 396:21, 1998.

[45] Mailman M, Schreck CF, OHern CS, and Chakraborty B. Jamming in systems composed of frictionless Ellipse-Shaped particles. Physical Review Letters, 102:255501, 2009.

[46] Majmudar TS, Sperl M, Luding S, and Behringer RP. Jamming transition in granular systems. Phys. Rev. Lett., 98:058001, 2007.

[47] Makse HA and Kurchan J. Testing the thermodynamic approach to granular matter with a numerical model of a decisive experiment. $\mathrm{Na}$ ture, 415(6872):614, 2002.

[48] Meyer F. Topographic distance and watershed lines. Signal processing, 38(1):113, 1994.

[49] Motl J. Bradley local image thresholding. http: //www.mathworks.com/matlabcentral/fileexchange/ 40854-bradley-local-image-thresholding. Updated: 2013-06-26. 
[50] Natterer F and Ritman EL. Past and future directions in x-ray computed tomography (ct). International Journal of Imaging Systems and Technology, 12(4):175, 2002.

[51] Neudecker M. Data from: Jammed frictional tetrahedra are hyperstatic. http://dx.doi.org/10.1103/PhysRevLett.111.028001.

[52] Neudecker M, Ulrich S, Herminghaus S, and Schröter M. Jammed frictional tetrahedra are hyperstatic. Phys. Rev. Lett., 111:028001, 2013. doi:10.1103/PhysRevLett.111.028001. URL http://link.aps . org/doi/10.1103/PhysRevLett.111.028001.

[53] Niblack W. An introduction to Digital Image Processing. Prentice Hall, 1986.

[54] Nicolas M, Duru P, and Pouliquen O. Compaction of a granular material under cyclic shear. The European Physical Journal E, 3(4):309, 2000.

[55] Nowak ER, Knight JB, Ben-Naim E, Jaeger HM, and Nagel SR. Density fluctuations in vibrated granular materials. Phys. Rev. E, 57:1971, 1998. doi:10.1103/PhysRevE.57.1971.

[56] Ohser J and Schladitz K. 3D images of materials structures: processing and analysis. John Wiley \& Sons, 2009.

[57] Otsu N. A threshold selection method from gray-level histograms. Automatica, 11(285-296):23, 1975.

[58] OHern CS, Silbert LE, Liu AJ, and Nagel SR. Jamming at zero temperature and zero applied stress: The epitome of disorder. Physical Review E, 68(1):011306, 2003.

[59] Philipse AP. The random contact equation and its implications for (colloidal) rods in packings, suspensions, and anisotropic powders. Langmuir, 12(5):1127, 1996.

[60] Pouliquen O, Nicolas M, and Weidman P. Crystallization of non-brownian spheres under horizontal shaking. Phys. Rev. Lett., 79(19):3640, 1997.

[61] Pournin L and Liebling T. Which regular spherotetrahedra best fill space? Zetta (EPFL notes), pages 52-56, 2012.

[62] Pournin L, Ramaioli M, Folly P, and Liebling TM. About the influence of friction and polydispersity on the jamming behavior of bead assemblies. The European Physical Journal E, 23(2):229, 2007. 
[63] Pournin L, Weber M, Tsukahara M, Ferrez JA, Ramaioli M, and Liebling TM. Three-dimensional distinct element simulation of spherocylinder crystallization. Granular Matter, 7(2-3):119, 2005.

[64] Press WH. Numerical recipes 3rd edition: The art of scientific computing. Cambridge university press, 2007.

[65] Radon J. 1.1 über die Bestimmung von Funktionen durch ihre Integralwerte längs gewisser Mannigfaltigkeiten. Classic papers in modern diagnostic radiology, page 5, 2005.

[66] Ribiere P, Richard P, Philippe P, Bideau D, and Delannay R. On the existence of stationary states during granular compaction. The European Physical Journal E, 22:249-253, 2007. ISSN 1292-8941. 10.1140/epje/e2007-00017-x.

[67] Richard P, Nicodemi M, Delannay R, Ribiere P, and Bideau D. Slow relaxation and compaction of granular systems. Nature materials, 4(2):121, 2005.

[68] Richard P, Philippe P, Barbe F, Bourlès S, Thibault X, and Bideau D. Analysis by x-ray microtomography of a granular packing undergoing compaction. Phys. Rev. E, 68(2):020301, 2003.

[69] Roerdink JB and Meijster A. The watershed transform: Definitions, algorithms and parallelization strategies. Fundamenta Informaticae, 41(1):187, 2000.

[70] Röntgen WC. Über eine neue Art von Strahlen. Annalen der Physik, 300(1):1, 1898.

[71] Schaller FM, Kapfer SC, Evans ME, Hoffmann MJ, Aste T, Saadatfar M, Mecke K, Delaney GW, and Schröder-Turk GE. Set voronoi diagrams of $3 \mathrm{~d}$ assemblies of aspherical particles. Philosophical Magazine, (ahead-of-print):1, 2013.

[72] Schaller FM, Neudecker M, Saadatfar M, Delaney G, Mecke K, Schröder-Turk GE, and Schröter M. Tomographic analysis of jammed ellipsoid packings. AIP Conf. Proceedings, 1542:377, 2013.

[73] Schröter M, Nägle S, Radin C, and Swinney H. Phase transition in a static granular system. Europhysics Letters, 78(4):44004, 2007.

[74] Schulze D. Pulver und Schüttguter: Fließeigenschaften und Handhabung. 2006.

[75] Shafait F, Keysers D, and Breuel TM. Efficient implementation of local adaptive thresholding techniques using integral images. $D R R$, 6815:681510, 2008. 
[76] Shui-Xiang L, Lu P, Jin W, and Meng L. Quasi-random packing of tetrahedra. Soft Matter, 2013.

[77] Shundyak K, van Hecke M, and van Saarloos W. Force mobilization and generalized isostaticity in jammed packings of frictional grains. Phys. Rev. E, 75:010301, 2007. doi:10.1103/PhysRevE.75.010301.

[78] Silbert LE, Liu AJ, and Nagel SR. Structural signatures of the unjamming transition at zero temperature. Physical Review E, 73(4):041304, 2006 .

[79] Smith KC, Alam M, and Fisher TS. Athermal jamming of soft frictionless platonic solids. Phys. Rev. E, 82:051304, 2010. doi: 10.1103/PhysRevE.82.051304. URL http://link.aps.org/doi/10. 1103/PhysRevE. 82.051304.

[80] Smith KC, Fisher TS, and Alam M. Isostaticity of constraints in amorphous jammed systems of soft frictionless platonic solids. Phys. Rev. E, 84:030301, 2011. doi:10.1103/PhysRevE.84.030301.

[81] Soille P. Morphological Image Analysis. Springer Berlin / Heidelberg, 2004.

[82] Song C, Wang P, and Makse HA. A phase diagram for jammed matter. Nature, 453(7195):629, 2008.

[83] Stock SR, editor. Comparison between X-ray tube based and synchrotron radiation based $C T$, volume 7078. SPIE, SPIE Press, 2008.

[84] Tetrapak. Tetrapak photos. http://www.flickr.com/photos/ tetrapak/6498150583/in/photostream/. Accessed 2013-10-24.

[85] Torquato S and Jiao Y. Dense packings of polyhedra: Platonic and archimedean solids. Phys. Rev. E, 80(4):041104, 2009.

[86] Torquato S and Jiao Y. Dense packings of the platonic and archimedean solids. Nature, 460(460):876-879, 2009.

[87] Torquato S and Jiao Y. Exact constructions of a family of dense periodic packings of tetrahedra. Phys. Rev. E, 81:041310, 2010. doi:10.1103/ PhysRevE.81.041310.

[88] Torquato S, Truskett TM, and Debenedetti PG. Is random close packing of spheres well defined? Phys. Rev. Lett., 84:2064, 2000. doi: 10.1103/PhysRevLett.84.2064. URL http://link.aps.org/doi/10. 1103/PhysRevLett. 84.2064. 
[89] Trier OD and Taxt T. Evaluation of binarization methods for document images. IEEE Transactions on Pattern Analysis and Machine Intelligence, 17:312, 1995.

[90] van Hecke M. Jamming of soft particles: geometry, mechanics, scaling and isostaticity. J. Phys. Condens. Matter, 22:033101, 2010.

[91] Vanhamel I, Pratikakis I, and Sahli H. Multiscale gradient watersheds of color images. IEEE Transactions on Image Processing, 12(6):617, 2003.

[92] Vyssotsky VA, Gordon SB, Frisch HL, and Hammersley JM. Critical percolation probabilities (bond problem). Phys. Rev., 123:1566, 1961. doi:10.1103/PhysRev.123.1566. URL http://link.aps.org/doi/10. 1103/PhysRev.123.1566.

[93] Waser R. Nanoelectronics and information technology. Wiley-VCH, Berlin, 2012.

[94] Wassenberg J, Middelmann W, and Sanders P. An efficient parallel algorithm for graph-based image segmentation. page 1003, 2009.

[95] Wirjadi O. Survey of 3d image segmentation methods. Technical Report 123, Fraunhofer ITWM, 2007.

[96] Zhao J, Li S, Jin W, and Zhou X. Shape effects on the random-packing density of tetrahedral particles. Phys. Rev. E, 86:031307, 2012. doi: 10.1103/PhysRevE.86.031307. URL http://link.aps.org/doi/10. 1103/PhysRevE.86.031307.

[97] Zhao SC and Schröter M. Measuring the configurational temperature of a binary disc packing. arXiv preprint arXiv:1302.6987, 2013. 


\section{Appendix D}

\section{Acknowledgements}

Ich danke meinem Doktorvater Matthias für die kontinuierliche Unterstützung während der Promotion, beim Überzeugen des PRL-Editors, bei zahlreichen Verlängerungsanträgen und der Elternzeit und für die Möglichkeit, zahlreiche Konferenzen von Dresden bis Dallas zu besuchen.

Ich danke meinen Ko-Autoren Dr. Stephan Ulrich und Prof. Dr. Stephan Herminghaus, sowie meinen Kollegen im "Spaghetti"-Projekt: Claus Heussinger, Sebastian Pitikaris, Christian Brosowski und Cyprian Lewandowski.

Thanks to my colleagues from the MPIDS, among others: Annika for introduction to the Nanotom, Christian "the gardener" for keeping an eye on our poor plants, Daniel for numerous espressi, sympathy and pizza, Fabian for many helpful discussions on image analysis and practical help with the shaker, my office-mate Julie for a great working atmosphere at any time, Nirmal Thyagu for support in experiments and analysis, Sonia for a handmade tetrahedral mug.

Meiner Frau Dörte für ihre Liebe, ihre Geduld und ihr Verständnis!

Meinen Eltern, die mich immer unterstützt haben.

Und zu guter Letzt danke ich Albert Camus dafür, dass er mir eine neue Perspektive auf Sisyphus eröffnet hat. 


\section{Appendix E}

\section{Publications}

Neudecker M, Ulrich S, Herminghaus S, and Schröter M. Jammed frictional tetrahedra are hyperstatic. Phys. Rev. Lett., 111:028001, 2013. doi:10.1103/PhysRevLett.111.028001

Schaller FM, Neudecker M, Saadatfar M, Delaney G, Mecke K, SchröderTurk GE, and Schröter M. Tomographic analysis of jammed ellipsoid packings. AIP Conf. Proceedings, 1542:377, 2013. http://dx.doi.org/10. $1063 / 1.4811946$

Steinmeier, U, Neudecker, M, von Hoersten, D and Schröter, M. Segregation of RFID Markers in Wheat, Transactions of the ASABE (2013), submitted, under review 